\title{
1 Conserved cell types with divergent features between human and mouse cortex
}

2 Rebecca D Hodge ${ }^{* 1}$, Trygve E Bakken ${ }^{* 1}$, Jeremy A Miller ${ }^{1}$, Kimberly A Smith ${ }^{1}$, Eliza R Barkan ${ }^{1}$, 3 Lucas T Graybuck ${ }^{1}$, Jennie L Close ${ }^{1}$, Brian Long ${ }^{1}$, Osnat Penn ${ }^{1}$, Zizhen Yao ${ }^{1}$, Jeroen

4 Eggermont ${ }^{2}$, Thomas Hollt ${ }^{2,3}$, Boaz $\mathrm{P}$ Levi $^{1}$, Soraya I Shehata ${ }^{1}$, Brian Aevermann ${ }^{4}$, Allison

5 Beller ${ }^{5}$, Darren Bertagnolli ${ }^{1}$, Krissy Brouner ${ }^{1}$, Tamara Casper $^{1}$, Charles Cobbs ${ }^{6}$, Rachel Dalley ${ }^{1}$, 6 Nick Dee ${ }^{1}$, Song-Lin Ding ${ }^{1}$, Richard G Ellenbogen ${ }^{7}$, Olivia Fong ${ }^{1}$, Emma Garren ${ }^{1}$, Jeff Goldy ${ }^{1}$,

7 Ryder P Gwinn ${ }^{8}$, Daniel Hirschstein ${ }^{1}$, C Dirk Keene ${ }^{5}$, Mohamed Keshk ${ }^{4}$, Andrew L Ko ${ }^{7,9}$, Kanan

8 Lathia $^{1}$, Ahmed Mahfouz ${ }^{2,3}$, Zoe Maltzer ${ }^{1}$, Medea McGraw ${ }^{1}$, Thuc Nghi Nguyen ${ }^{1}$, Julie Nyhus ${ }^{1}$,

9 Jeffrey G Ojemann ${ }^{7,9}$, Aaron Oldre ${ }^{1}$, Sheana Parry ${ }^{1}$, Shannon Reynolds ${ }^{1}$, Christine Rimorin ${ }^{1}$,

10 Nadiya V Shapovalova ${ }^{1}$, Saroja Somasundaram ${ }^{1}$, Aaron Szafer ${ }^{1}$, Elliot R Thomsen ${ }^{1}$, Michael

11 Tieu $^{1}$, Richard H Scheuermann ${ }^{4,10}$, Rafael Yuste ${ }^{11}$, Susan M Sunkin ${ }^{1}$, Boudewijn Lelieveldt ${ }^{2,3}$,

12 David Feng ${ }^{1}$, Lydia Ng${ }^{1}$, Amy Bernard ${ }^{1}$, Michael Hawrylycz ${ }^{1}$, John W. Phillips ${ }^{1}$, Bosiljka Tasic ${ }^{1}$,

13 Hongkui Zeng ${ }^{1}$, Allan R Jones ${ }^{1}$, Christof Koch ${ }^{1}$, Ed S Lein ${ }^{\# 1}$

${ }^{1}$ Allen Institute for Brain Science, Seattle, WA, USA

${ }^{2}$ Department of Radiology, Leiden University Medical Center, Leiden, The Netherlands

$17{ }^{3}$ Department of Intelligent Systems, Delft University of Technology, Delft, the Netherlands

184 J. Craig Venter Institute, La Jolla, CA, USA

$19{ }^{5}$ Department of Pathology, University of Washington, Seattle, WA, USA

$20{ }^{6}$ The Ben and Catherine Ivy Center for Advanced Brain Tumor Treatment, Swedish

21 Neuroscience Institute, Seattle, WA, USA

$22{ }^{7}$ Department of Neurological Surgery, University of Washington School of Medicine, Seattle,

23 WA

$24{ }^{8}$ Epilepsy Surgery and Functional Neurosurgery, Swedish Neuroscience Institute, Seattle, WA,

25 USA

$26{ }^{9}$ Regional Epilepsy Center at Harborview Medical Center, Seattle, WA, USA

$27{ }^{10}$ Department of Pathology, University of California, San Diego, CA, USA

$28{ }^{11}$ Neurotechnology Center, Department of Biological Sciences, Columbia University, New York,

29 NY, USA

30

31

* Contributed equally

$32 \quad$ \#Correspondence should be addressed to Ed S. Lein (edl@alleninstitute.org) 


\section{Abstract}

34 Elucidating the cellular architecture of the human neocortex is central to understanding our

35 cognitive abilities and susceptibility to disease. Here we applied single nucleus RNA-

36 sequencing to perform a comprehensive analysis of cell types in the middle temporal gyrus of

37 human cerebral cortex. We identify a highly diverse set of excitatory and inhibitory neuronal

38 types that are mostly sparse, with excitatory types being less layer-restricted than expected.

39 Comparison to a similar mouse cortex single cell RNA-sequencing dataset revealed a

40 surprisingly well-conserved cellular architecture that enables matching of homologous types and

41 predictions of human cell type properties. Despite this general conservation, we also find

42 extensive differences between homologous human and mouse cell types, including dramatic

43 alterations in proportions, laminar distributions, gene expression, and morphology. These

44 species-specific features emphasize the importance of directly studying human brain.

\section{Introduction}

46 The cerebral cortex, responsible for most of our higher cognitive abilities, is the most complex structure known to biology and is comprised of approximately 16 billion neurons and 61 billion non-neuronal cells organized into approximately 200 distinct anatomical or functional regions $s^{1,2,3,4}$. The human cortex is greatly expanded relative to the mouse, the dominant model organism in basic and translational research, with a 1200 -fold increase in cortical neurons compared to only a 60 -fold increase in sub-cortical neurons (excluding cerebellum) ${ }^{5,6}$. The general principles of neocortical development and the basic multilayered cellular cytoarchitecture of the neocortex appear relatively conserved across mammals ${ }^{7,8}$. However, whether the cellular and circuit architecture of cortex is fundamentally conserved across mammals, with a massive evolutionary areal expansion of a canonical columnar architecture in human, or is qualitatively and quantitatively specialized in human, remains an open question long debated in the field ${ }^{9,10}$. Addressing this question has been challenging due to a lack of tools to broadly characterize cell type diversity in complex brain regions, particularly in human brain tissues.

Prior studies have described differences in the cellular makeup of the cortex in human and specialized features of specific cell types ${ }^{11,12,13,14,15,16,17}$, although the literature is remarkably limited. For example, the supragranular layers of cortex, involved in cortico-cortical communication, are differentially expanded in mammalian evolution ${ }^{18}$. Furthermore, certain cell types show highly specialized features in human and non-human primate compared to mouse, such as the interlaminar astrocytes ${ }^{17}$, and the recently described rosehip cell ${ }^{19}$, a type of inhibitory interneuron in cortical layer 1 with distinctive morpho-electrical properties. All of these cellular properties are a function of the genes that are actively used in each cell type, and transcriptomic methods provide a powerful method to understand the molecular underpinnings of cellular phenotypes as well as a means for mechanistic understanding of species-specialized phenotypes. Indeed, a number of studies have shown significant differences in transcriptional regulation between mouse, non-human primate and human, including many genes associated with neuronal structure and function $20,21,22,23$.

Dramatic advances in single cell transcriptional profiling present a new approach for large-scale comprehensive molecular classification of cell types in complex tissues, and a metric for comparative analyses. The power of these methods is fueling ambitious new efforts to understand the complete cellular makeup of the mouse brain ${ }^{24}$ and the even the whole human body ${ }^{25}$. Recent applications of single cell RNA-sequencing (scRNA-seq) methods in mouse

78 cortex have demonstrated robust transcriptional signatures of neuronal and non-neuronal cell 
types ${ }^{26,27,28}$, and suggest the presence of approximately 100 neuronal and non-neuronal cell types in any given cortical area. Similar application of scRNA-seq to human brain has been challenging due to the difficulty in dissociating intact cells from densely interconnected human tissue $^{29}$. In contrast, single nucleus RNA-sequencing (snRNA-seq) methods allow for transcriptional profiling of intact neuronal nuclei that are relatively easy to isolate and enable use of frozen postmortem specimens from human brain repositories ${ }^{30,31,32}$. Importantly, it was recently shown that single nuclei contain sufficient gene expression information to distinguish closely related subtypes of cells at a similar resolution to scRNA-seq ${ }^{33,34}$, demonstrating that snRNA-seq is a viable method for surveying cell types that can be compared to scRNA-seq data. Early applications of snRNA-seq to human cortex demonstrated the feasibility of the approach but have not provided depth of coverage sufficient to achieve similar resolution to mouse studies ${ }^{35}$.

The current study aimed to establish a robust methodology for relatively unbiased cell type classification in human brain using snRNA-seq, and to perform the first comprehensive comparative analysis of cortical cell types to understand conserved and divergent features of human and mouse cerebral cortex. We first describe the cellular landscape of the human cortex, and then demonstrate a similar degree of cellular diversity between human and mouse and a conserved set of homologous cell types and subclasses. In contrast, we present evidence for extensive differences between homologous types, including evolutionary changes in relative proportions, laminar distributions, subtype diversity, gene expression and other cellular phenotypes.

\section{Results}

\section{Transcriptomic taxonomy of cell types}

102 A robust snRNA-seq methodology was established to analyze transcriptomically defined cell

103

104

105

106

107

108

109

110

111

112

113

114 types in human cortex. We focused on the middle temporal gyrus (MTG), with samples largely derived from high-quality postmortem brain specimens. This region is frequently available through epilepsy surgery resections, permitting a comparison of postmortem versus acute neurosurgical tissues, as well as allowing future correlation with in vitro slice physiology experiments in MTG. Frozen tissue blocks were thawed, vibratome sectioned, and stained with fluorescent Nissl dye. Individual cortical layers were microdissected, tissues were homogenized to release nuclei, and nuclei were stained with an antibody against NeuN to differentiate neuronal (NeuN-positive) and non-neuronal (NeuN-negative) nuclei. Single nuclei were collected via fluorescence-activated cell sorting (FACS) (Fig. 1A, Extended Data Figure 1A, Methods). We sorted $\sim 90 \%$ NeuN-positive and $\sim 10 \%$ NeuN-negative nuclei across all cortical layers to enrich for neurons. The final dataset contained less than the targeted $10 \%$ nonneuronal nuclei because nearly $50 \%$ of NeuN-negative nuclei failed quality control criteria, potentially due to the lower RNA content of glia compared to neurons (Methods) ${ }^{27}$. SMARTSeqv4 (Takara Bio USA Inc.) was used to reverse transcribe mRNA and amplify cDNA. Sequencing libraries were generated using Nextera XT (Illumina), which were sequenced on a HiSeq 2500 at a median depth of $2.6+/-0.5$ million reads/nucleus. Nuclei were collected from 8 total human tissue donors (4 male, 4 female; 4 postmortem, 4 neurosurgical) ranging in age

120 from 24 to 66 years (Extended Data Table 1). 15,206 nuclei were collected from postmortem 121 tissue donors with no history of neuropathology or neuropsychiatric disorders, and 722 nuclei 122 came from apparently histologically normal MTG distal to pathological tissue that was removed 123 during surgical resections to treat epilepsy (Methods). 
124 To evenly survey cell type diversity across cortical layers, nuclei were sampled based on the

125

126

127

128

129

130

131

132

133

134

135

136

137

138

139

140

141

142

143

144

145

146

147

148

149

150

151

152

153

154

155

156

157

158

159

160

161

162

163

164

165

166

167

168

169

170

171

172

relative proportion of neurons in each layer reported in human temporal cortex ${ }^{36}$. Based on Monte Carlo simulations, we estimated that 14,000 neuronal nuclei were needed to target types as rare as $0.2 \%$ of the total neuron population (Methods). Using an initial subset of RNA-seq data, we observed more transcriptomic diversity in layers 1, 5, and 6 than in other layers so additional neuronal nuclei were sampled from those layers. In total, 15,928 nuclei passed quality control criteria and were split into three broad classes of cells (10,708 excitatory neurons, 4297 inhibitory neurons, and 923 non-neuronal cells) based on NeuN staining and cell class marker gene expression (Methods).

Nuclei from each broad class were iteratively clustered as described in ${ }^{33}$. Briefly, high variance genes were identified while accounting for gene dropouts, expression dimensionality was reduced with principal components analysis (PCA), and nuclei were clustered using JaccardLouvain community detection (Methods). On average, neuronal nuclei were larger than nonneuronal nuclei (Extended Data Fig. 1B), and median gene detection (Extended Data Fig. 1C,D) was correspondingly higher for neurons (9046 genes) than for non-neuronal cells (6432 genes), as previously reported for mouse ${ }^{26,27,28}$. Transcriptomic cell types were largely conserved across diverse individuals and tissue types (postmortem, neurosurgical), since all curated clusters contained nuclei derived from multiple donors, and nuclei from postmortem and neurosurgical tissue types clustered together (Fig. 1B, Extended Data Fig. 2A). However, a small, but consistent expression signature related to tissue type was apparent; for example, nuclei derived from neurosurgical tissues exhibited higher expression of some activity related genes (Extended Data Fig. 2). 325 nuclei were assigned to donor-specific or outlier clusters that contained marginal quality nuclei and were excluded from further analysis (Methods).

This analysis method defined 75 transcriptomically distinct cell types, including 45 inhibitory neuron types that express the canonical GABAergic interneuron marker GAD1, 24 excitatory neuron types that express the vesicular glutamate transporter SLC17A7, and 6 non-neuronal types that express the glutamate transporter SLC1A3 (Fig. 1C, D). As expected based on prior studies $26,27,28,31$, the hierarchical relationships among types roughly mirrors the developmental origin of different cell types. We refer to the cell type clusters as cell types, intermediate order nodes as subclasses, and higher order nodes such as the interneurons derived from the caudal ganglionic eminence (CGE) as classes, and the broadest divisions such as excitatory neurons as major classes. Neuronal types split into two major classes representing cortical plate-derived glutamatergic excitatory neurons ( $n=10,525$ nuclei) and ganglionic eminence-derived GABAergic inhibitory neurons ( $n=4164$ nuclei). Non-neuronal types ( $n=914$ nuclei) formed a separate main branch based on differential expression of many genes (Fig. 1C). We developed a principled nomenclature for clusters based on: 1) major cell class, 2) layer enrichment (including layers containing at least $10 \%$ of nuclei in that cluster), 3 ) a subclass marker gene (maximal expression of 14 manually-curated genes), and 4) a cluster-specific marker gene (maximal detection difference compared to all other clusters) (Fig. 1D, Extended Data Fig. 3, Methods). For example, the left-most inhibitory neuron type in Figure 1D, found in samples dissected from layers 1 and 2, and expressing the subclass marker PAX6 and the specific marker $C D H 12$, is named Inh L1-2 PAX CDH12. Additionally, we generated a searchable semantic representation of these cell type clusters that incorporates this accumulated knowledge about marker gene expression, layer enrichment, specimen source, and parent cell class to link them to existing anatomical and cell type ontologies ${ }^{37}$ (Supplementary Data). We find broad correspondence to an earlier study ${ }^{31}$, but identify many additional types of excitatory and inhibitory neurons due to increased sampling and/or methodological differences (Extended Data Fig. 4). The majority of cell types were rare ( $<100$ nuclei per cluster, $<0.7 \%$ of cortical 
173

174

175

176

177

178

179

180

181

182

183

184

185

186

187

188

189

190

191

192

193

194

195

196

197

198

199

200

201

202

203

204

205

206

207

208

209

210

211

212

213

214

215

216

217

218

219

220

neurons), including almost all interneuron types and deep layer excitatory neuron types. In contrast, the excitatory neurons of superficial layers 2-4 were dominated by a small number of relatively abundant types (>500 nuclei per cluster, $>3.5 \%$ of neurons) (Fig. 1C). Both excitatory types and many interneuron types were restricted to a few layers, whereas non-neuronal nuclei were distributed across all layers, with the notable exception of one astrocyte type (Fig. 1C).

\section{Excitatory neurons often span multiple layers}

The 24 transcriptionally distinct excitatory neuron types broadly segregated by layer and expressed known laminar markers (Fig. 2A-C). In general, excitatory types were most similar to other types in the same or adjacent layers. Transcriptomic similarity by proximity for cortical layers has been described before, and interpreted as a developmental imprint of the inside-out generation of cortical layers ${ }^{38}$. Complex relationships between clusters are represented as constellation diagrams (Fig. 2A, Methods) ${ }^{26}$, where the circles represent core cells that were most transcriptionally similar to the cluster to which they were originally assigned, and indicate the size (proportional to circle area) and average laminar position of each cell type. The thickness of lines between cell clusters represents their similarity based on the number of nuclei whose assignment to a cluster switched upon reassignment (intermediate cells, Methods). This similarity by proximity is also apparent in the hierarchical dendrogram structure of cluster similarity in Figure 2B. One exception is the layer 5 Exc L5-6 THEMIS C1QL3 type, which has a transcriptional signature similar to layer 2 and 3 types as well as several deep layer cell types (Fig. 2A, B). Two types, Exc L4-5 FEZF2 SCN4B and Exc L4-6 FEZF2 IL26, were so distinct that they occupied separate branches on the dendrogram and did not connect via intermediate cells to any other type (Fig. 2A, B).

Each excitatory type showed selective expression of genes that can be used as cell type markers (Fig. 2C), although in general a small combinatorial profile (generally 2-3 genes per type) was necessary to distinguish each type from all other cortical cell types (Fig. 2D). The majority of these markers are novel as excitatory neuron markers, and belonged to diverse and functionally important gene families, such as BHLH transcription factors (TWIST2), collagens (COL22A1), and semaphorins (SEMA3E). Surprisingly, 16 out of $37(41 \%)$ of these most specific marker genes were unannotated loci (LOCs), long non-coding RNAs (lincRNA), pseudogenes, and antisense transcripts. This may partially be a result of profiling nuclear RNA, as some of these transcripts have been shown to be enriched in the nucleus (Fig. 2C, Extended Data Figs. 3, 5) ${ }^{39}$.

Unexpectedly, most excitatory neuron types were present in multiple layers based on layer dissection information (Fig. 2B). Within the supragranular layers, three main types were enriched in layer 2 and 3 dissections. Additionally, ten RORB-expressing types were enriched in layer 3-6 dissections (Fig. 2B, C). Layers 5 and 6 contained 11 excitatory types: 4 types that expressed THEMIS (Thymocyte Selection Associated), 6 types that expressed FEZF2, and 1 type that expressed the cytokine IL15 (Interleukin 15). The majority of these types were similarly represented in layer 5 and 6 dissections (Fig. 2B). To clarify whether this crossing of layer boundaries was an artifact of dissection or a feature of MTG organization, we investigated the layer distribution of 10 types using multiplex fluorescence in situ hybridization (FISH) with combinatorial gene panels designed to discriminate clusters (Fig. 2B, D, Extended Data Fig. 6). In situ distributions largely validated snRNA-seq predictions (Fig. 2E). Three types were mainly localized to layer $3 \mathrm{c}$ and the upper part of layer 4 , defined as the dense band of granule cells visible in Nissl stained sections (Fig 2E). Interestingly, one of these types (Exc L3-4 RORB CARM1P1) had large nuclei, suggesting that it may correspond to a subset of the giant pyramidal layer $3 \mathrm{c}$ neurons previously described in MTG ${ }^{40}$ (Fig. 2E, Extended Data Fig. 6). 
Two types were mostly restricted to layer 4 (Exc L3-5 RORB ESR1, Exc L4-5 RORB DAPK2), but the five other types examined all spanned multiple layers (Fig. 2E). Taken together, the snRNA-seq and in situ validation data indicate that transcriptomically defined excitatory neuron types are frequently not layer-specific, but rather spread across multiple anatomically defined layers.

\section{Heterogeneous expression within clusters}

A major evolutionary feature of human cortical architecture is the expansion of supragranular layers compared to other mammals, and morphological and physiological properties of pyramidal neurons vary across layers 2 and 3 of human temporal cortex ${ }^{40,41}$. In that light, it was surprising to find only three main excitatory clusters in human cortical layers 2 and 3 . However, one cluster was very large (Exc L2-3 LINC00507 FREM3; $\mathrm{n}=2284$ nuclei) and spanned layers 2 and 3 , posing the possibility that there is significant within-cluster heterogeneity. Indeed, we find continuous variation in gene expression in this cluster along the axis of cortical depth, illustrated well using two data visualization and mining tools built for this project to allow public access to this dataset. The Cytosplore MTG Viewer (https://viewer.cytosplore.org), is an extension of Cytosplore ${ }^{42}$, and presents a hierarchy of t-SNE maps of different subsets of MTG clusters ${ }^{43}$, with each map defined using informative marker genes (Fig. 3A). Layer dissection metadata overlaid onto the t-SNE map of Exc L2-3 LINC00507 FREM3 revealed that nuclei in this type were ordered by layer, with nuclei sampled from layers 2 and 3 occupying relatively distinct locations in t-SNE space. Selecting nuclei at both ends of the cluster gradient in t-SNE space and computing differential expression between these nuclei revealed a set of genes with variable expression across this cluster (Fig. 3A, Supplementary Movie 1). Data Navigator (http://celltypes.brain-map.org/rnaseq/human) showed gradient expression between layers 2 and 3 (Fig. 3B). Finally, single molecule FISH confirmed gradient expression of $L A M P 5$ and COL5A2 across layers 2 and 3 in cells mapping to this cluster (Fig. 3C,

Extended Data Figs. 7, 8). These results illustrate that there is additional diversity in human supragranular pyramidal neurons manifested as continuous variation in gene expression as a function of cortical depth that likely correlates with anatomical and functional heterogeneity of those cells.

\section{Inhibitory neuron diversity}

252 GABAergic inhibitory neurons split into two major branches, largely distinguished by expression of Adenosine Deaminase, RNA Specific B2 (ADARB2) and the transcription factor LIM Homeobox 6 (LHX6) (Fig. 4A-F). In mouse cortex, interneurons split into the same two major branches, also defined by expression of Adarb2 and $L h x 6$ and developmental origins in the caudal ganglionic eminence (CGE) and medial ganglionic eminence (MGE), respectively ${ }^{26}$. The ADARB2 branch was further subdivided into the LAMP5/PAX6 and VIP subclasses of interneurons, with likely developmental origins in the CGE. Surprisingly, the serotonin receptor subunit $H T R 3 A$, which marks CGE-derived interneurons in mouse ${ }^{44}$, was not a good marker of these types in human (Fig. 4E). The LHX6 branch consisted of PVALB and SST subclasses of interneurons, likely originating in the medial ganglionic eminence MGE ${ }^{45,46}$. Consistent with mouse cortex ${ }^{26}$, the $A D A R B 2$ branch showed a much higher degree of diversity in supragranular layers 1-3 compared to layers 4-6, whereas the opposite was true for the $L H X 6$ branch (Fig. 4A, B). As with the excitatory neuron taxonomy, many interneuron cluster specific markers were unannotated (LOC) genes, lincRNAs, pseudogenes, and antisense transcripts (Fig. 4E, F). 
The LAMP5/PAX6 subclass of interneurons included 6 transcriptomic types, many of which were enriched in layers 1 and 2 (Fig. 4C). Several types coexpressed SST (Fig. 4E), consistent with previous reports demonstrating SST expression in layer 1 of human MTG ${ }^{19}$ and different from mouse Lamp5 and Pax6 interneurons ${ }^{26,27}$, which do not express SST. The Inh L1-4 LAMP5 LCP2 type expressed marker genes of rosehip cells, a type of interneuron with characteristic large axonal boutons that we described in a previous study of layer 1 MTG interneurons ${ }^{19}$. With whole cortex coverage, it is clear that this type is not restricted to layer 1 but rather present across all cortical layers. Among LAMP5/PAX6 types on the ADARB2 (CGEderived) branch, Inh L2-6 LAMP5 CA1 cells uniquely expressed $L H X 6$, suggesting possible developmental origins in the MGE, and appear similar to the Lamp5 Lhx6 cells previously described in mouse cortex ${ }^{26,27}$.

VIP interneurons represented the most diverse subclass, containing 21 transcriptomic types (Fig. 4A), many of which were enriched in layers 2 and 3 (Fig. 4C). Several types in the VIP subclass (Inh L1 SST CHRNA4 and Inh L1-2 SST BAGE2) appeared to be closely related to the L1 SST NMBR type of the LAMP5/PAX6 subclass, as evidenced by intermediate cell connections between these types. Interestingly, these highly related types were all localized to layers 1 and 2. Furthermore, while both the Inh L1 SST CHRNA4 and Inh L1-2 SST $B A G E 2$ were grouped into the VIP subclass, they appeared to lack expression of VIP. Rather, they expressed SST, consistent with expression of this gene in layer 1 and 2 interneurons as discussed above (Fig. 4A, C, E) ${ }^{19}$. The Inh L1-2 GAD1 MC4R type also lacked expression of VIP (Fig. 4E). Notably, this type specifically expresses the Melanocortin 4 Receptor, a gene linked to autosomal dominant obesity and previously shown to be expressed in a population of mouse hypothalamic neurons that regulate feeding behavior ${ }^{48,49}$.

The SST subclass consisted of 11 transcriptomic types, including one highly distinct type, Inh L3-6 SST NPY, that occupied its own discrete branch on the dendrogram and was not connected to other types in the SST constellation (Fig. 4B, D). Several SST types displayed laminar enrichments, with Inh L5-6 SST TH cells being a particularly restricted type, found only in layers 5 and 6 . We further validated marker gene expression and the spatial distribution of the Inh L3-6 SST NPY and Inh L5-6 SST TH types using ISH from the Allen Human Brain Atlas (http://human.brain-map.org/; Fig. 4G). ISH for TH confirmed that expression of this gene is sparse and restricted to layers 5-6; interestingly, Th ISH in mouse temporal association area (TEa; the closest homolog to human MTG) showed similar sparse labeling restricted to layers 5 and 6 , suggesting that this gene may mark similar cell types in human and mouse (http://mouse.brain-map.org/; Fig. 4G). In contrast, the well-known interneuron marker neuropeptide $Y(N p y)$ was broadly expressed in a scattered pattern throughout all layers in mouse TEa, whereas, in human MTG, NPY labeled only a single interneuron type whose sparsity was confirmed by ISH (Fig. 4G), indicating that this heavily-studied marker labels a different cohort of cell types in human and mouse ${ }^{50,51}$.

The PVALB subclass comprised 7 clusters, including two types that were grouped into this branch but did not appear to express PVALB (Fig. 4F). One of these types, Inh L5-6 SST MIR548F2, had low expression of SST, whereas the other type, Inh L5-6 GAD1 GLP1R, did not express any canonical interneuron subclass markers. Intermediate cells connected the Inh L5-6 SST MIR548F2 type in the PVALB constellation to the Inh L5-6 SST TH type in the SST constellation. Two other connections between the SST and PVALB constellations were apparent, both of which included the Inh L2-4 SST FRZB cluster (Fig. 4B). One highly distinctive PVALB type (Inh L2-5 PVALB SCUBE3) (Fig. 4B, D) likely corresponds to chandelier (axo-axonic) cells as it expresses UNC5B, a marker of chandelier (axo-axonic) cells in mouse ${ }^{52}$ (Fig. 4H). Multiplex FISH (RNAscope, Methods) validated expression of several novel marker 
genes (NOG, COL15A1, Fig. 4H) and showed enrichment of these cells mainly in layers 2-4, consistent with the pattern observed in the snRNA-seq data (Fig. 4D, H).

\section{Diverse morphology of astrocyte types}

319 Although non-neuronal (NeuN-) cells were not sampled as deeply as neurons, all major glial types - astrocytes, oligodendrocytes, endothelial cells, and microglia - were identified (Fig. 5A). In contrast to studies of mouse cortex where non-neuronal cells were more extensively sampled or selectively targeted with Cre lines ${ }^{26,28,53}$, we did not find other types of immune or vascular cells. This decreased diversity is likely largely due to more limited non-neuronal sampling, but may also reflect the age of tissue analyzed. For example, previous reports showed that adult mouse cortex contains mainly oligodendrocyte progenitor cells (OPCs) and mature oligodendrocytes, but few immature and myelinating oligodendrocyte types ${ }^{28,53}$, similarly, we found only two oligodendrocyte types, one of which expressed markers of oligodendrocyte progenitor cells (OPCs) (e.g. PDGFRA, OLIG2) and another that expressed mature oligodendrocyte markers (e.g. OPALIN, MAG) (Fig. 5A, B).

Astrocytes in human cortex are both functionally ${ }^{54}$ and morphologically ${ }^{17}$ specialized in comparison to rodent astrocytes, with distinct morphological types residing in different layers of human cortex (Fig. 5C). Interlaminar astrocytes, described only in primates to date, reside in layer 1 and extend long processes into lower layers, whereas protoplasmic astrocytes are found throughout cortical layers 2-6 ${ }^{17}$ (Fig.5C). Similarly, we find two astrocyte clusters with different laminar distributions. Astro L1-2 FGFR3 GFAP originated mostly from layer 1 and 2 dissections, whereas the Astro L1-6 FGFR3 SLC14A1 type was found in all layers (Fig.5A). The two astrocyte types we identified were distinguished by expression of the specific marker gene ID3 along with higher expression of GFAP and AQP4 in the Astro L1-2 FGFR3 GFAP type than in the Astro L1-6 FGFR3 SLC14A1 type (Fig. 5B, D). To determine if these two transcriptomic types correspond to distinct morphological types, we labeled cells with a combination of multiplex FISH and immunohistochemistry for GFAP protein. Cells with high GFAP and AQP4 expression, characteristic of the Astro L1-2 FGFR3 GFAP type and consistent with previous reports of interlaminar astrocytes ${ }^{55}$, were present predominantly in the upper half of layer 1 (Fig. 5E). Coexpression of $A Q P 4$ and ID3 was apparent in layer 1 cells that had extensive, long-ranging GFAP-positive processes characteristic of interlaminar astrocytes (Fig. 5E). In contrast, GFAP-positive cells with protoplasmic astrocyte morphology lacked expression of ID3, consistent with the Astro L1-6 FGFR3 SLC14A1 type (Fig. 5E). layer 1 and 2 dissections, seven nuclei were from layer 5 and 6 dissections and expressed ID3 as well as a distinct set of marker genes (Fig. 5D). Based on their laminar origin, we hypothesized that these nuclei may correspond to fibrous astrocytes, which are enriched in white matter ${ }^{17}$ (Fig. 5C). Indeed, astrocytes at the border of layer 6 and the underlying white matter coexpressed ID3 and AQP4 and had relatively thick, straight GFAP-positive processes characteristic of fibrous astrocytes (Fig. 5E), suggesting that the Astro L1-6 FGFR3 GFAP cluster contains a mixture of two different morphological astrocyte types. Given that nuclei corresponding to fibrous astrocytes express distinct marker genes from interlaminar astrocytes (Fig. 5D), it is likely that fibrous astrocytes will form a separate transcriptomic type

360 with increased sampling. 


\section{Human and mouse cell type homology}

362 Single cell transcriptomics not only provides a new method for comprehensive analysis of species-specific cellular diversity, but also a quantitative metric for comparative analysis between species. Furthermore, identification of homologous cell types or classes allows inference of cellular properties from much more heavily studied model organisms. The availability of densely sampled single cell or single nucleus RNA-seq datasets in human (described here) and mouse ${ }^{26}$ cortex using the same RNA-seq profiling platform allowed a direct comparison of transcriptomic cell types. The success of such a comparison is predicated on the idea of conserved transcriptional patterning. As a starting point, we asked whether the same types of genes discriminate human interneuron cell types as those reported for mouse interneuron types ${ }^{52}$. Indeed, we find the same sets of genes (mean $=21$ genes $/$ set) best discriminate human interneuron types (Fig.6A), including genes central to neuronal connectivity and signaling. Similar functional classes of genes also discriminate human and mouse excitatory neuron types (although with less conservation for classes of genes that discriminate non-neuronal cell types; Extended Data Fig.9A), indicating that shared expression patterns between species may facilitate matching cell types.

Simply combining expression data for inhibitory neuron nuclei from human MTG and for cells from mouse V1 was not sufficient for identification of homologous cell types. PCA analysis resulted in samples clearly separated by species along the first principal component that explained almost $20 \%$ of expression variation (Fig.6B, Extended Data Fig.9B). Recent work has demonstrated the power of canonical correlation analysis (CCA) to align single cell RNAseq data from human and mouse based on shared co-expression patterns ${ }^{56}$. Application of CCA and graph-based clustering to human and mouse cortical samples was much more successful (Fig.6B), and allowed matching of human and mouse types based on shared CCA cluster membership for inhibitory neurons (Fig.6C, Extended Data Fig.9E), excitatory neurons (Fig.6D, Extended Data Fig.9F) and non-neuronal cells (Fig.6E, Extended Data Fig.9G).

Remarkably, shared co-expression between mouse V1 and human MTG enabled the identification of homologous types at approximately half the resolution of the full human classification (38 types versus 75 types). Combining the CCA results allowed generation of a hierarchical taxonomy including 34 neuronal and 4 non-neuronal cell types and subclasses (Fig. $6 F$ ). A hybrid nomenclature from human and mouse ${ }^{27}$ was used to describe these homologous types. Ten cell types were matched one-to-one between species, whereas other types were matched at a subclass resolution. Transcriptomically distinct cell types more often had one-toone matches, likely because more redundant marker genes compensated for divergent expression patterns, and we find even most rare types had homologous types in mouse and human.

408

This homology alignment enabled prediction of the anatomical, functional, and connectional properties of human cell types based on the much larger mouse literature for homologous cell types. For example, the human cluster Inh L2-5 PVALB SCUBE3 described above matches one-to-one with the mouse chandelier (or axo-axonic) cell type Pvalb Vipr2, suggesting that this cell type selectively innervates the axon initial segment of excitatory neurons. Also, the human cluster Inh L3-6 SST NPY matches the mouse Sst Chodl type and is therefore predicted to have long-range projections and contribute to sleep regulation ${ }^{26,57,58}$. Many other anatomically defined interneuron types could be similarly inferred, including basket, Martinotti, bipolar, neurogliaform, and single-bouquet cells (Fig. 6C), although future experiments will be necessary to confirm these predictions. 
The long-range projection targets of human glutamatergic neurons (e.g. intratelencephalic (IT), pyramidal tract (PT), and corticothalamic (CT)) that would otherwise be experimentally inaccessible can also be inferred based on their best transcriptomic match to mouse cell types; for example, the human Exc L4-5 FEZF2 SCN4B type corresponds to the PT sub-cortically projecting layer 5 pyramidal cells (Fig. 6D). The Exc L4-6 FEZF2 IL26 matches two mouse layer 5 types (L5 NP S/c17a8 and L5 NP Rapgef3) that lack long-range projections ${ }^{26,59}$. Finally, layer $6 \mathrm{~b}$ (subplate) types can be identified by homology, and among human layer $6 \mathrm{~b}$ types, Exc L6 FEZF2 OR2T8 has much larger nuclei (Extended Data Fig. 1B) and corresponds to the mouse L6b Rprm type that selectively projects to thalamus rather than cortex.

Four of five human non-neuronal cell types matchedmouse cell types (Fig. 6E), while endothelial cells had such divergent global expression patterns between species that they could not be matched by CCA despite the expression of conserved canonical marker genes (e.g. EMCN and NOSTRIN). The mouse Oligo Enpp6 cluster partially overlapped nuclei from human OPC and mature oligodendrocyte clusters and appears to represent an immature oligodendrocyte type ${ }^{26,53}$ that is rare or not present in adult human cortex. The morphologically distinct human layer 1 astrocyte type, Astro L1-2 FGFR3 GFAP, did not match any clusters from ${ }^{26}$, although a layer 1 enriched astrocyte with shared marker gene expression was previously reported in mouse ${ }^{28}$. Finally, while the majority of human microglia clustered with mouse microglia, two nuclei clustered with mouse perivascular macrophages (Extended Data Fig.9D), suggesting that this rare type was likely undersampled in human.

Only three mouse neuronal types and two human interneuron types lacked homologous types, although all three mouse types are very rare and may not have been sampled in human. The mouse Meis2 inhibitory type, which is primarily restricted to white matter and has an embryonic origin outside of the ganglionic eminence ${ }^{26}$, may have been missed due to limited sampling of layer $6 \mathrm{~b}$ and underlying white matter. Mouse Cajal-Retzius cells are glutamatergic neurons in layer 1 . These cells are exceedingly rare (less than $0.1 \%$ of layer 1 neurons) in adult human cortex ${ }^{60,61}$ and were not expected to be sampled. Finally, the mouse layer 5 pyramidal tract type L5 PT Chrna6, a rare excitatory neuron type with strong projections to superior colliculus ${ }^{59}$, has no matching human cluster. However, 2 of 25 nuclei from the human pyramidal tract (PT)-like cluster Exc L4-5 FEZF2 SCN4B are more similar to this distinct mouse PT type than to other mouse PT types (Extended Data Fig.9C), suggesting this mismatch is also due to undersampling in human. Interestingly, both human interneuron types that lack closely matched mouse homologues (Inh L1 SST CHRNA4 and Inh L1-2 GAD1 MC4R) are highly enriched in layer 1 . Along with the phenotypic specialization of the layer 1 rosehip neuron ${ }^{19}$, it appears that layer 1 may be a hotspot of evolutionary change at the level of inhibitory cell types.

447 While many homologous subclasses had comparable diversity between species, some subclasses had expanded diversity in human or mouse. Human layer 4 excitatory neurons are more diverse than those of mouse (Fig. 6D), contributing to increased diversity of supragranular layers due to mixing into layer 3 as described above. Mouse layer 5 PT types are much more diverse than those in human, which may reflect either a true species difference or undersampling, as they make up $<1 \%$ of layer 5 excitatory neurons in human MTG. Layer 6 CT types also show greater diversity in mouse V1 than human MTG; however, this difference may reflect an areal difference between a primary sensory area that has strong, reciprocal connnections with the thalamus and an area of association cortex. Indeed, we find increased diversity of cell types in human visual cortex that match mouse layer 6 CT types (data not shown). 


\section{Divergent proportions of cell types}

459 Alterations in the relative proportions of cell types could have profound consequences for 460 cortical circuit function. snRNA-seq data predicted a significant species difference in the 461 proportions of interneuron classes. Human MTG showed similar proportions of MGE-derived (44\% LHX6+ nuclei) and CGE-derived (50\% ADARB2+ nuclei) interneurons, whereas in mouse cortex roughly $70 \%$ of interneurons are MGE-derived and $\sim 30 \%$ are CGE-derived ${ }^{44,62}$. To validate these differences, we applied multiplex FISH to quantify the proportions of CGE $(A D A R B 2+)$ and MGE (LHX6+) interneurons in human MTG and mouse TEa (Fig. 7, Extended Data Fig. 10). Interneurons that co-expressed $A D A R B 2$ and $L H X 6$, corresponding to the human Inh L2-6 LAMP5 CA1 and mouse Lamp5 Lhx6 types (Figs. 1, 4), were considered separately. Consistent with the snRNA-seq data, we found similar proportions of MGE $(50.2 \pm 2.3 \%)$ and CGE (44.2 $\pm 2.4 \%$ ) interneurons in human MTG, whereas we found more than twice as many MGE $(67.8 \pm 0.9 \%)$ than CGE $(30.8 \pm 1.2 \%)$ interneurons in mouse TEa. The increased proportion of CGE-derived interneurons in human was greatest in layer 4 , whereas the decreased proportion of MGE interneurons in human was greatest in layers 4-6 (Fig. 7A). Interestingly, both the snRNA-seq data (6.1\% of GAD1+ cells) and in situ cell counts (5.6 \pm $0.3 \%$ of GAD1+ cells) confirmed a significant increase in the proportion of the Inh L2-6 LAMP5 CA1 type in human MTG versus the Lamp5 Lhx6 type in mouse TEa $(1.4 \pm 0.2 \%$ of GAD1+ cells), most notably in layer 6 (Fig. 7A).

Another major predicted mismatch was seen for the sub-cortically projecting PT neurons, which comprise approximately $20 \%$ of layer 5 excitatory neurons in mouse but less than $1 \%$ in human based on single cell ${ }^{26}$ and single nucleus RNA-seq sampling. To directly compare the spatial distribution and abundance of PT types between species, we performed ISH for a pan-layer 5 PT marker (Fam84b) ${ }^{26}$ in mouse TEa and for markers of the homologous layer 5 PT type Exc L4-5 FEZF2 SCN4B in human MTG. In mouse TEa, Fam84b was expressed in many neurons performed triple FISH with the pan-excitatory marker SLC17A7, the PT markers FAM84B or POU3F1, and NPTX1, which labels most SLC17A7-positive layer 5 neurons but not PT cells (Fig. 7B, Extended Data Fig. 11). In MTG, SLC17A7+/NPTX1- cells co-labeled with $F A M 84 B$ or POU3F1 were sparsely distributed predominantly in superficial layer 5 and were large with a prominent, thick apical dendrite (Fig. 7B, Extended Data Fig. 11). Thus, PT cells have a similar distribution within layer 5 in human and mouse but are much less abundant in human, likely reflecting an evolutionary scaling constraint as discussed below.

\section{Divergent expression between homologous types}

493 The identification of homologous or consensus cell types or classes allows direct analysis of the conservation and divergence of gene expression patterns across these types. For each pair of homologous cell types, we compared expression levels of 14,414 orthologous genes between human and mouse. Nuclear expression levels were estimated based on intronic reads to better compare human single nucleus and mouse single cell RNA-seq data. The Exc L3c/L5a type (Exc L3-4 RORB CARM1P1 in human) has the most conserved expression ( $r=0.78$ ) of all types, and yet $12 \%$ of genes have highly divergent expression (defined as $>10$-fold difference),

500 including many specific markers (orange dots, Fig. 8A) for this cell type. Microglia had the least

501

502

503

504 conserved expression $(r=0.60)$, and more than $20 \%$ of genes were highly divergent (Fig. 8B). Surprisingly, the Exc L3c/L5a consensus type shows a striking shift in layer position between human, where Exc L3-4 RORB CARM1P1 is highly enriched in layer 3c of MTG, and mouse, where the homologous type L5 Endou is enriched in layer 5 a of mouse V1 (Fig.

505 8A). This laminar shift of a homologous cell type helps explain the reported expression shift of 
several genes from layer 5 in mouse to layer 3 in human ${ }^{20}$, including two genes (BEND5 and PRSS12) expressed in Exc L3-4 RORB CARM1P1 but not in layer 3 of mouse TEa.

Over half of all genes analyzed (8222, or $57 \%$ ) had highly divergent expression in at least one of the 38 homologous types, and many genes had divergent expression restricted to a specific

511

512 cell type or broad class (Fig. 8C). Non-neuronal cell types had the most highly divergent

513 expression including 2025 genes with >10-fold species difference, supporting increased evolutionary divergence of non-neuronal expression patterns between human and mouse brain described previously ${ }^{22}$.

Most genes had divergent expression in a subset of types rather than all types, and this resulted in a shift in the cell type specificity or patterning of genes. These expression pattern changes were quantified as the beta score of log-fold differences across cell types

(Methods, Supplementary Table 2), and scores were approximately log-normally distributed with a long tail of highly divergent genes (Fig. 8D). Cell type marker genes tended to be less conserved than more commonly expressed genes (Fig. 8E). In many cases, the most defining markers for cell types were not shared between human and mouse. For example, chandelier interneurons selectively express Vipr2 in mouse but COL15A1 and NOG in human (Fig. 4H). Interestingly, the functional classes of genes that best differentiate cell types within a species (Fig. 6A) are the same functional classes that show the most divergent expression patterns between species (Fig. 8F). In other words, the same gene families show cell type specificity in both species, but their patterning across cell types frequently differs.

The top 20 most divergent gene families between human and mouse (i.e. highest median pattern change) include neurotransmitter receptors (serotonin, adrenergic, glutamate, peptides, and glycine), ion channels (chloride), and cell adhesion molecules involved in axonal pathfinding (netrins and cadherins). Among the top 3\% most divergent genes (see Supplementary Table 2 for full list), the extracellular matrix collagens COL24A1 and COL12A1 and the glutamate receptor subunits GRIK1 and GRIN3A were expressed in different cell types between species and were validated by ISH to have different laminar distributions in human MTG and mouse TEa (Fig. 8G). The cumulative effect of so many differences in the cellular patterning of genes with well characterized roles in neuronal signaling and connectivity is certain to cause many differences in human cortical circuit function.

\section{Discussion}

539 Single cell transcriptomics provides a powerful tool to systematically characterize the cellular 540 diversity of complex brain tissues, allowing a paradigm shift in neuroscience from the historical emphasis on cellular anatomy to a molecular classification of cell types and the genetic blueprints underlying the properties of each cell type. Echoing early anatomical studies ${ }^{10}$, recent studies of mouse neocortex have shown a great diversity of cell types ${ }^{26,28}$. Similar studies of human cortex ${ }^{35,31,32}$ have shown the same broad classes of cells but much less subtype diversity (Extended Data Fig. 4), likely resulting from technical differences, such as fewer nuclei sampled or reduced gene detection. A recent study showed a high degree of cellular diversity in human cortical layer $1^{19}$ by densely sampling high-quality postmortem human tissue with snRNA-seq and including intronic sequence to capture signal in nuclear transcripts ${ }^{33}$. The current study takes a similar dense sampling approach by sequencing approximately 16,000 single nuclei spanning all cortical layers of MTG, and defines 75 cell types

551 representing non-neuronal (6), excitatory (24) and inhibitory (45) neuronal types. Importantly, 
robust cell typing could be achieved despite the increased biological and technical variability between human individuals. Nuclei from postmortem and acute surgically resected samples clustered together, and all clusters described contained nuclei from multiple individuals. Importantly, the ability to use these methods to study the fine cellular architecture of the human brain and to identify homologous cell types based on gene expression allows inference of cellular phenotypes across species as well. In particular, since so much knowledge has been accumulated about the cellular makeup of rodent cortex based on transcriptomics, physiology, anatomy and connectivity, this approach immediately allows strong predictions about such features as well as others that are not currently possible to measure in human such as developmental origins and long-range projection targets.

This molecular paradigm can help unify the field and increase the cellular resolution of many studies but has several consequences and challenges. Unambiguous definition of transcriptomic cell types in situ typically requires the detection of two or more markers with multiplexed molecular methods, demonstrating the need to further develop spatial transcriptomics methods ${ }^{63}$. Developing consistent nomenclature will also be challenging, particularly when marker genes are not conserved across species. Establishing cell type homologies across species can generate hypotheses about conserved and divergent cell features, and facilitates the larger, open access efforts to profile single cells across the brain underway in mouse, monkey, and human through the BRAIN Initiative ${ }^{24}$ and the Human Cell Atlas ${ }^{25}$. The current data are made publicly available with two new viewer applications to mine expression data across transcriptomic cell types in both human and mouse cortex (www.brainmap.org; viewer.cytosplore.org).

Interestingly, whereas excitatory neuron types are traditionally referred to as being confined to a single cortical layer, we find instead that many transcriptomically-defined excitatory types are represented in multiple layers. In part, this may reflect indistinct laminar boundaries in MTG; for example, von Economo ${ }^{40}$ noted intermixing of granule and pyramidal neurons in layer 4 along with blending of layer 4 pyramidal neurons into adjacent layers 3 and 5 in MTG. However, we find several types with broad spatial distributions across multiple layer boundaries, suggesting that indistinct laminar boundaries do not fully account for this lack of strict laminar segregation. Examination of the spatial distribution of excitatory neuron types in additional cortical areas will be necessary to determine if this is a particular feature of MTG or a more widespread

584 phenomenon in human cortex.

The transcriptomic cellular organization and diversity in human MTG are surprisingly similar to those of mouse $\mathrm{V} 1{ }^{26}$, despite many differences in these data sets. First, mouse scRNA-seq was compared to human snRNA-seq, and to mitigate this, expression levels were estimated using intronic sequence that should be almost exclusively retained in the nucleus ${ }^{33}$. Second, young adult ( $~ 8$-week-old) mice were compared to older (24-66 years) human specimens; however, prior transcriptomic studies demonstrated stable gene expression throughout adulthood in human ${ }^{64,65}$. Third, MTG in human was compared to V1 in mouse. This areal difference is expected to primarily affect comparison of excitatory neurons that vary more between regions than inhibitory neurons or glia ${ }^{26}$. Finally, scRNA-seq introduces significant biases due to differential survival of cell types during dissociation, necessitating the use of Crelines to enrich for under-sampled and rare cell types in mouse cortex ${ }^{26}$. In contrast, we found that snRNA-seq provides more unbiased sampling and estimates of cell type proportions. Despite these differences, the human and mouse cell type taxonomies could be matched at high resolution and reveal a "canonical" cellular architecture that is conserved between cortical 600 areas and species. Beyond similarities in overall diversity and hierarchical organization, 10 cell 
601

602

603

604

605

606

607

608

609

610

611

612

613

614

615

616

617

618

619

620

621

622

623

624

625

626

627

628

629

630

631

632

633

634

635

636

637

638

639

640

641

642

643

644

645

646

647

types could be unambiguously mapped one-to-one between species, and 28 additional subclasses could be mapped at a higher level in the taxonomic tree. One-to-one matches were highly distinctive cell types, including several non-neuronal and neuronal types, such as chandelier cells. Comparison of absolute numbers of types between studies is challenging, but no major classes have missing homologous types other than exceedingly rare types that were likely undersampled in human, such as Cajal-Retzius cells.

A striking feature of cortical evolution is the relative expansion of the supragranular layers involved in cortico-cortical communication ${ }^{18}$. Consistent with this expansion, we find increased diversity of excitatory neurons in layers 2-4 in human compared to mouse. Layers 2 and 3 are dominated by three major types, but the most common layer $2 / 3$ type exhibits considerable transcriptomic heterogeneity in the form of gene expression gradients, which would be expected to correlate with other cellular phenotypes. We also find expanded diversity of excitatory types in deep layer 3 , along with a surprising increase in diversity in human layer 4 compared to mouse.

We observed several other evolutionary changes in cell type proportions and diversity that substantially alter the human cortical microcircuit. The relative proportions of major classes of GABAergic interneurons vary between human MTG and mouse V1, with human MTG having fewer PVALB- and SST-expressing interneurons and more LAMP5/PAX6- and VIP-expressing interneurons. Since these interneuron classes are derived from the MGE and CGE, respectively, in mouse, this difference is consistent with increased generation of CGE-derived interneurons in human ${ }^{45}$. Another major species difference is seen for human layer 5 excitatory neurons that are homologous to mouse sub-cortically projecting (PT) neurons. Both the frequency ( $<1 \%$ in human versus approximately $20 \%$ in mouse) and diversity ( 1 type in human versus 5 types in mouse) ${ }^{26}$ of PT neurons are markedly reduced in human, although reduced diversity may be an artifact of limited sampling in human. The sparsity of this type was confirmed in situ and was not a technical artifact of tissue processing. Rather, this sparsity likely reflects the 1200-fold expansion of human cortex relative to mouse compared to only 60 -fold expansion of sub-cortical regions that are targets of these neurons ${ }^{4,5}$. If the number of PT neurons scales with the number of their sub-cortical projection targets, then the 20 -fold greater expansion of cortical neurons would lead to a 20 -fold dilution of PT neuron frequency as we observed. Indeed, the number of human corticospinal neurons, a subset of sub-cortically projecting neurons, has scaled linearly with the number of target neurons in the spinal cord, both increasing 40 -fold compared to mouse ${ }^{66,67,68}$. Thus, this striking difference in cell type frequency may be a natural consequence of allometric scaling of the mammalian brain ${ }^{69}$.

Our results demonstrate striking species divergence of gene expression between homologous cell types, as observed in prior studies at the single gene ${ }^{20}$ or gross structural level ${ }^{21}$. We find more than half of all orthologous genes show a major (>10-fold) difference in expression in at least one of the 38 consensus cell types, and up to $20 \%$ of genes in any given cell type showing such major divergent expression. Several cell types, including endothelial cells, had such substantial expression divergence that they could not be matched across species using the methods employed here. These gene expression differences are likely to be functionally relevant, as divergent genes are associated with neuronal connectivity and signaling, signaling, including axon guidance genes, ion channels, and neuropeptide signaling. Surprisingly, serotonin receptors are the most divergent gene family, challenging the use of mouse models for the many neuropsychiatric disorders involving serotonin signaling ${ }^{70}$. Finally, the more selectively expressed a gene is in one species the less likely its pattern is to be conserved, and many well-known markers of specific cell types do not have conserved patterns. 
Homologous cell types can have highly divergent features in concert with divergent gene expression. Here, we show that the interlaminar astrocyte, which has dramatic morphological specialization in primates including human, corresponds to one of two transcriptomic astrocyte types. A recent scRNA-seq analysis of mouse cortex also found 2 types, with one enriched in layer $1^{28}$. However, this mouse astrocyte type had less complex morphology and did not extend the long-range processes characteristic of interlaminar astrocytes. Thus, a 10-fold increase in size, the formation of a long process, and other phenotypic differences ${ }^{17,55,54}$ are evolutionary variations on a conserved genetically defined cell type. Similarly, a recent study identified the rosehip interneuron in human layer 1 19, which showed species differences in anatomy, physiology and marker gene profiles suggesting that it is a novel type of interneuron in human cortex. In fact, we now find that this rosehip type can be mapped to a mouse neurogliaform interneuron type. Thus, phenotypic differences large enough to define cell types with conventional criteria represent relatively minor variation on a conserved genetic blueprint for

662 neurons as well.

663 Together these observations quantitatively frame the debate of whether human cortex is

664

665

666

667

668 different from that of other mammals ${ }^{9,10}$, revealing a basic transcriptomic similarity of cell types punctuated by differences in proportions and gene expression between species that could greatly influence microcircuit function. The current results help to resolve the seeming paradox of conserved structure across mammals but failures in the use of mouse for pre-clinical studies ${ }^{71,70}$, and they highlight the need to analyze the human brain in addition to model organisms. The magnitude of differences between human and mouse suggest that similar profiling of more closely related non-human primates will be necessary to study many aspects of human brain structure and function. The enhanced resolution afforded by these molecular technologies also has great promise for accelerating a mechanistic understanding of brain evolution and disease.

\section{Acknowledgements}

674 We would like to thank the Tissue Procurement, Tissue Processing, and Facilities teams at the Allen Institute for Brain Science for assistance with the transport and processing of postmortem and neurosurgical brain specimens. We thank the Technology team at the Allen Institute for assistance with data management. We gratefully acknowledge our collaborators at local hospitals (Swedish Medical Center, Harborview Medical Center/UW Medicine, and University of Washington Medical Center) for help with the coordination of human neurosurgical tissue collections. We thank Joe Davis and the San Diego Medical Examiner's Office for assistance

682

683

684

685

686

687 with postmortem tissue donations. We acknowledge the Molecular Biology, Histology, and Imaging teams at the Allen Institute for Brain Science for performing chromogenic in situ hybridization experiments. This work was funded by the Allen Institute for Brain Science, and by US National Institutes of Health grant 5 U01 MH114812-02 to E.S.L. Funding from NWO-AES projects 12721: 'Genes in Space' and 12720: 'VANPIRE' (P.I. Anna Vilanova) for development of the Cytosplore MTG Viewer is gratefully acknowledged. We thank Baldur van Lew for scripting and narration of Cytosplore instructional and use case videos. The authors thank the Allen Institute founder, Paul G. Allen, for his vision, encouragement, and support.

\section{Author Contributions}

\section{E.S.L conceptualized and supervised the study. E.S.L. and R.Y. conceptualized the Human Cell} Types Program. R.D.H and T.E.B. designed experiments. R.D.H., E.R.B., B. Long., J.L.C., B.P.L., S.I.S., K.B, J.G., D.H., S.L.D., M.M., S.P., E.R.T, N.V.S., and Z.M. contributed to nuclei isolation and/or validation experiments. T.E.B., J.A.M., O.P., Z.Y., O.F., J.G., S.S., and M.H. 
694 contributed to computational analyses. K.A.S. and B.T. managed the single-nucleus RNA-seq

695 pipeline. L.T.G. developed data visualization tools. B.T. and H.Z. provided the mouse cortex

696

697 transcriptomic cell type taxonomy for the cross-species comparative study. D.B., K.L., C.R, and M.T. performed single-nucleus RNA-seq. A. Bernard and J.P. managed establishment of singlenucleus RNA-seq pipeline. A. Bernard and M.M contributed to the development and management of histological methods and data generation. K.B. performed immunohistochemistry experiments. R.D., N.D., T.C., J.N., A.O. processed postmortem brain tissues. A. Bernard and N.D. managed acquisition of postmortem and neurosurgical tissues. A. Beller, C.D.K, C.C., R.G.E., R.P.G., A.L.K, and J.G.O. contributed to neurosurgical tissue collections. B.A., M.K., and R.H.S. developed the semantic representation of clusters. J.E., T.H., A.M., and B. Lelieveldt developed the Cytosplore MTG Viewer. L.T.G., J.A.M., D.F., L.N, and A. Bernard contributed to the development of the RNA-Seq Data Navigator. S.R., A.S., and S.M.S. provided program management and/or regulatory compliance support. C.K. and A.R.J. provided institutional support and project oversight. E.S.L. and H.Z. directed the Allen Institute Cell Types Program. R.D.H., T.E.B., and E.S.L. wrote the paper with contributions from J.A.M and J.L.C., and in consultation with all authors. 
bioRxiv preprint doi: https://doi org/10.1101/384826; this version posted Auqust 5, 2018. The copyright holder for this preprint (which was not certified by peer review) is the author/funder, who has granted bioRxiv a license to display the preprint in perpetuity. It is made available under aCC-BY-NC-ND 4.0 International license.

A

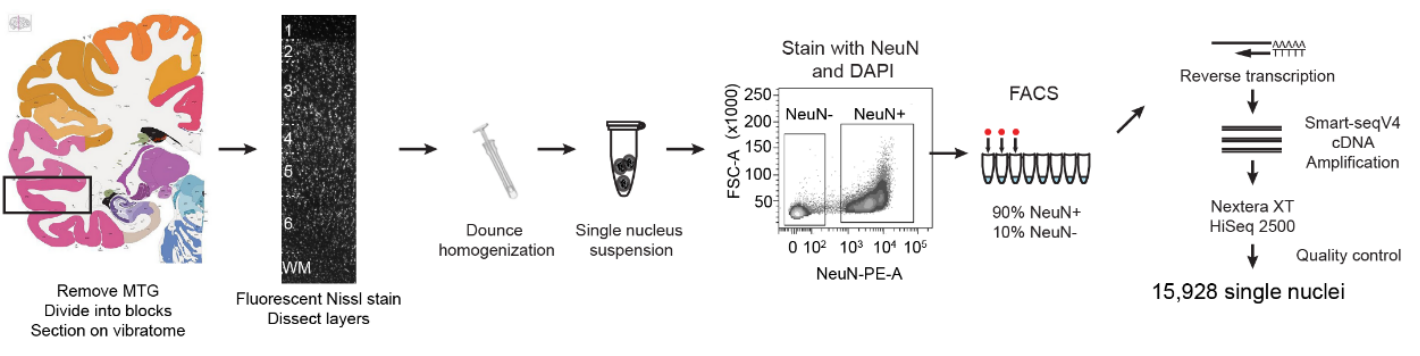

B
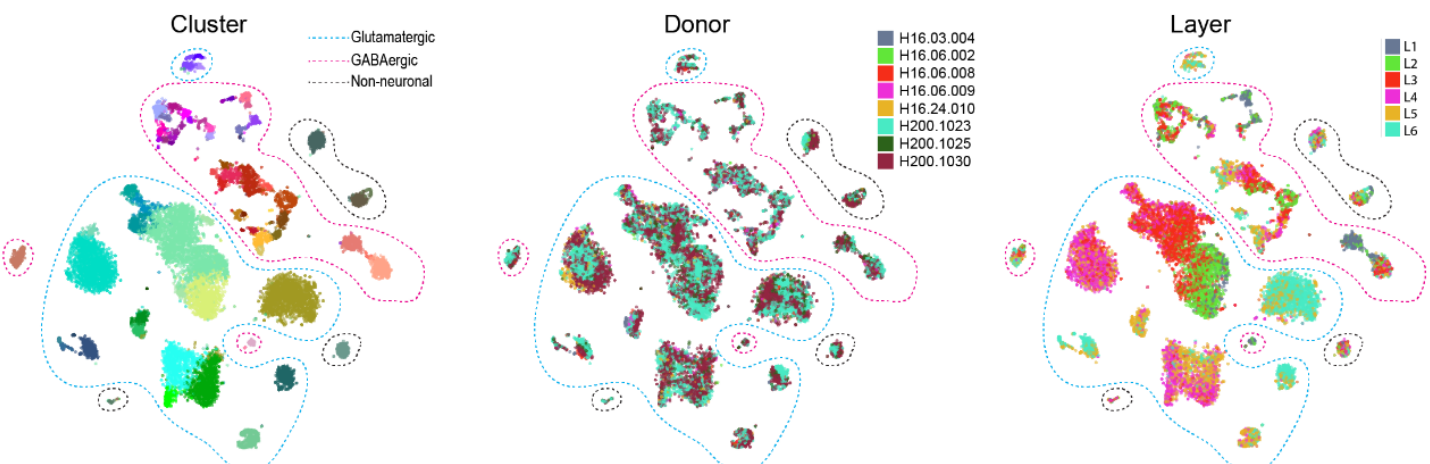

C

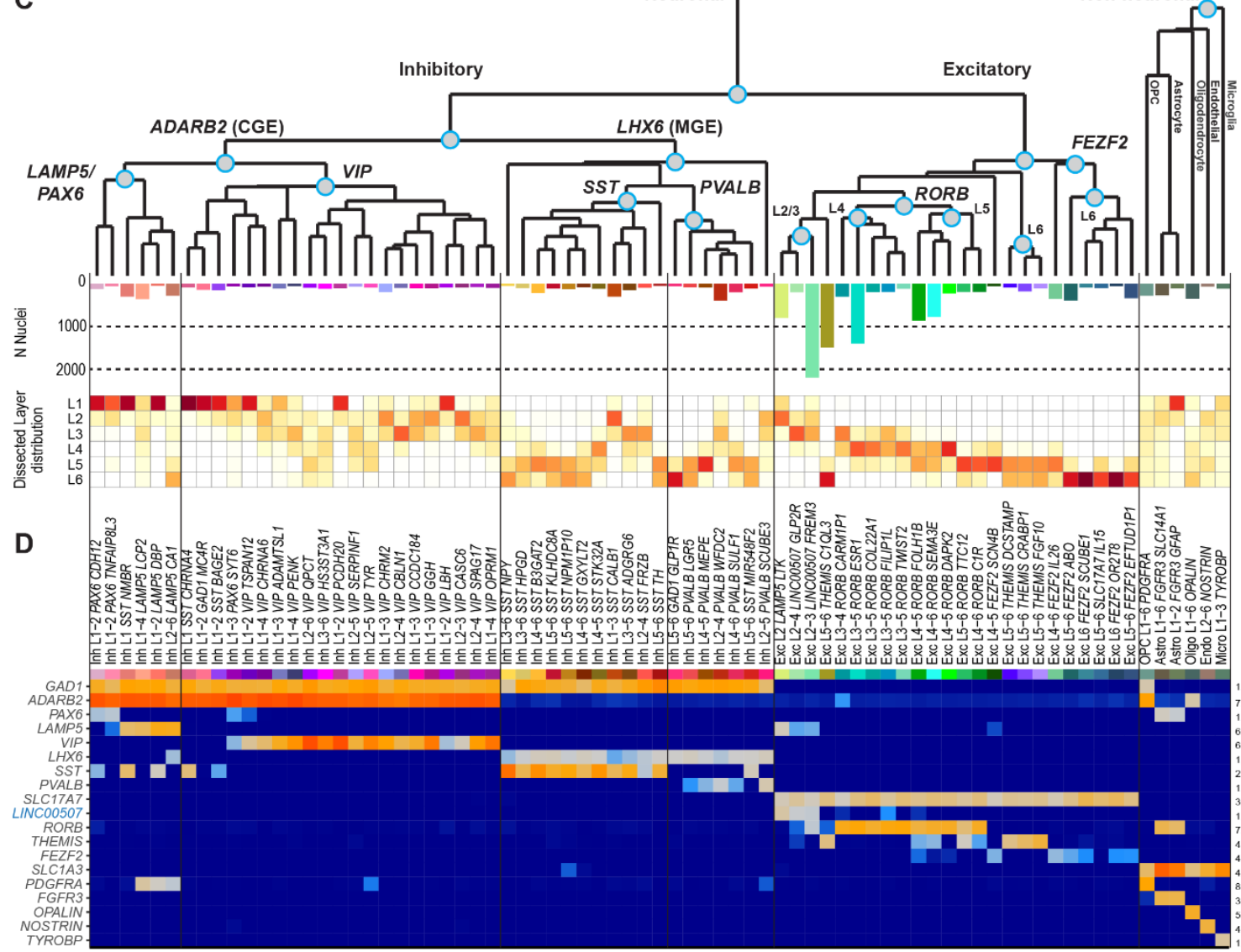


712 Figure 1. Cell type taxonomy in human middle temporal gyrus (MTG). (A) Schematic diagram illustrating nuclei isolation from frozen MTG specimens by vibratome sectioning, fluorescent Nissl staining and dissection of specific cortical layers. Single neuronal (NeuN+) and non-neuronal (NeuN-) nuclei were collected by fluorescence-activated cell sorting (FACS), and RNA-sequencing of single nuclei used SMART-seqv4, Nextera XT, and HiSeq2500 sequencing. (B) Overview of transcriptomic cell type clusters visualized using t-distributed stochastic neighbor embedding (t-SNE). On the left t-SNE map, each dot corresponding to one of 15,928 nuclei has a cell-type specific color that is used throughout the remainder of the manuscript. In the middle, donor metadata is overlaid on the t-SNE map to illustrate the contribution of nuclei from different individuals to each cluster. In the list of specimens, $\mathrm{H} 16.03 .004-\mathrm{H} 16.06 .009$ are neurosurgical tissue donors and $\mathrm{H} 16.24 .010-\mathrm{H} 200.1030$ are postmortem donors. On the right, layer metadata is overlaid on the t-SNE map to illustrate the laminar composition of each cluster. (C) Hierarchical taxonomy of cell types based on median cluster expression consisting of 69 neuronal (45 inhibitory, 24 excitatory) and 6 non-neuronal transcriptomic cells types. Major cell classes are labeled at branch points in the dendrogram. The bar plot below the dendrogram represents the number of nuclei within each cluster. The laminar distributions of clusters are shown in the plot that follows. For each cluster, the proportion of nuclei in each layer is depicted using a scale from white (low) to dark red (high). (D) Heatmap showing the expression of cell class marker genes (blue, non-coding) across clusters. Maximum expression values for each gene are listed on the far-right hand side. Gene expression values are quantified as counts per million of intronic plus exonic reads and displayed on a $\log _{10}$ scale. 
bioRxiv preprint doi: https://doi.org/10.1101/384826; this version posted August 5, 2018. The copyright holder for this preprint (which was not certified by peer review) is the author/funder, who has granted bioRxiv a license to display the preprint in perpetuity. It is made available under aCC-BY-NC-ND 4.0 International license.

A

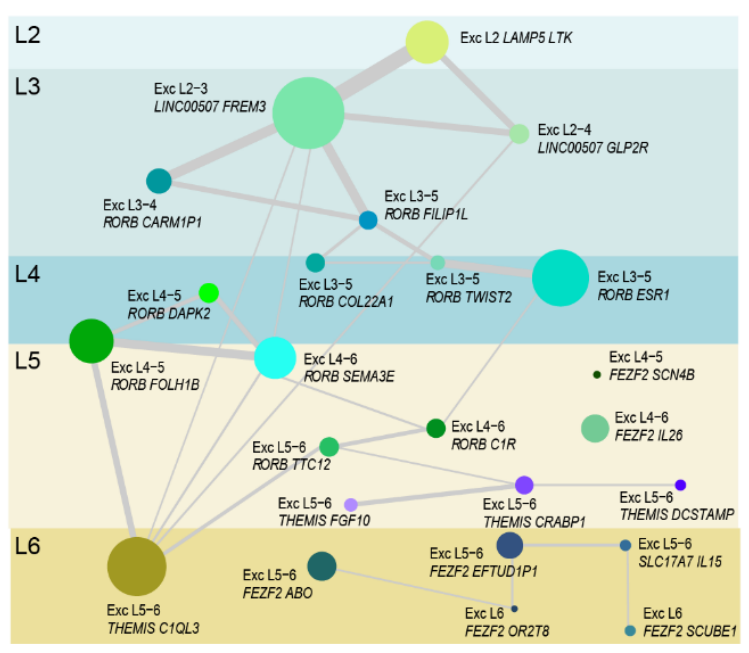

B

$$
\mathrm{L} 1
$$$$
\text { 㐫 }
$$

L6

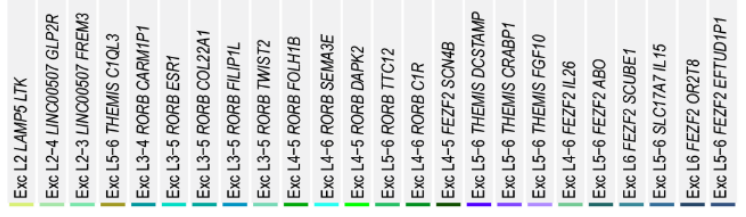

C

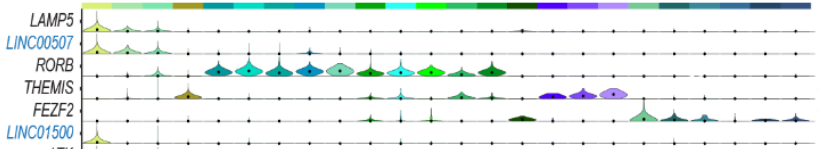

core $\quad$ D

50
250
500
1000

Intermediate

\begin{tabular}{l}
${ }_{10}^{3}$ \\
$=50$ \\
\hline \\
250
\end{tabular}

E
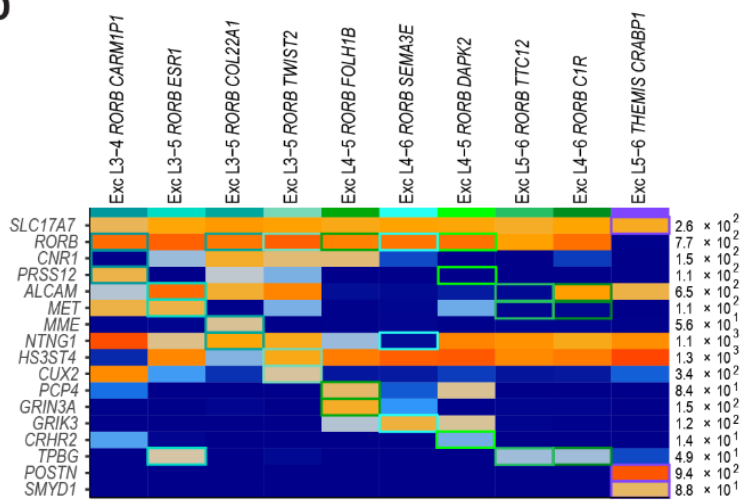

•RORB+ -TPBG+ - RORB+ - HS 3ST4+ -PCP4+ - GRIK3+ -CRHR2+ -TPBG+ -TPBG+ -SLC17A7+

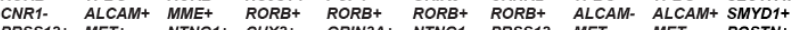

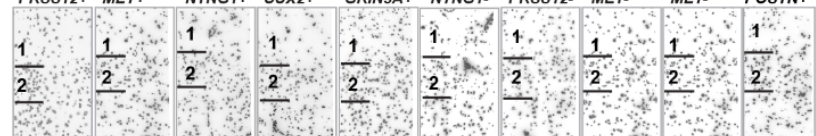

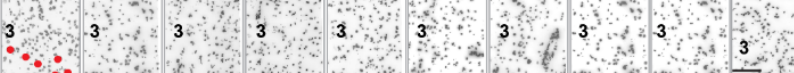

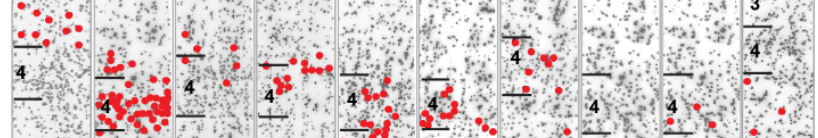

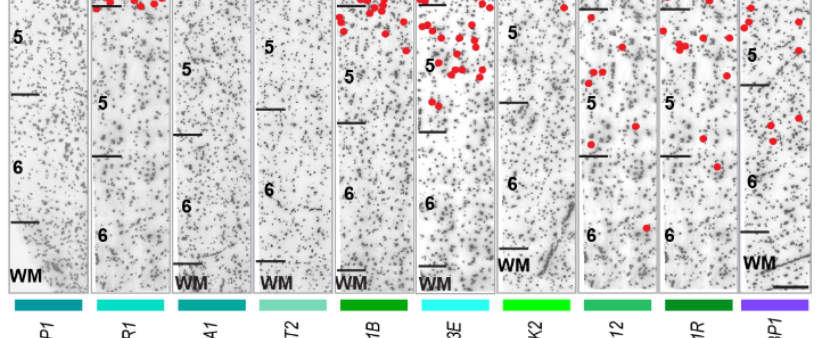

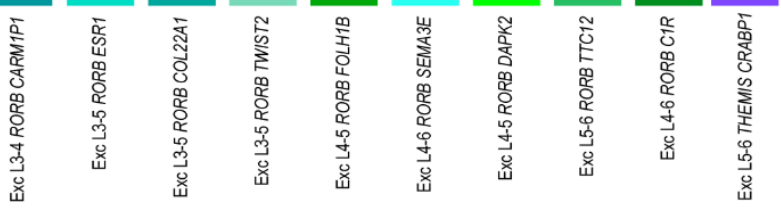

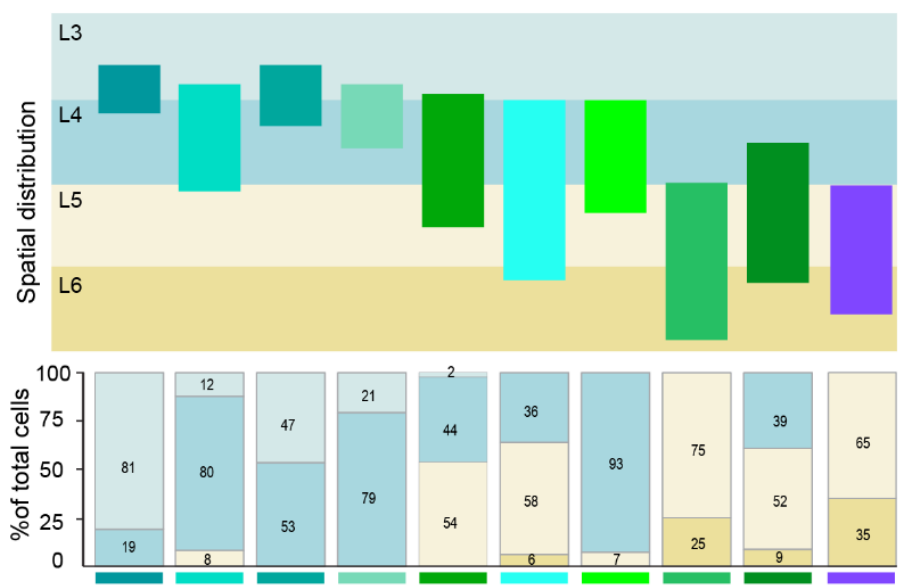


Figure 2. Excitatory neuron diversity and marker gene expression. (A) Constellation diagram for excitatory cell types. The number of cells that could be unambiguously assigned to each cluster (core cells) is represented by disc area and the number of cells with uncertain membership between each pair of clusters (intermediate cells) is represented by line thickness. (B) Dendrogram illustrating overall gene expression similarity between cell types. Layer distributions of cell types are shown as dot plots where each dot represents a single nucleus from a layer-specific dissection. Note that incidental capture of some layer 2 excitatory neurons occurred in layer 1 dissections and is reflected in the dot plots. Clusters marked by a red bar at the base of the dendrogram are examined using fluorescent in situ hybridization (FISH) in (D-E). (C) Violin plot showing marker gene (blue, non-coding) expression distributions across clusters. Each row represents a gene, black dots show median gene expression within clusters, and the maximum expression value for each gene is shown on the right-hand side of each row. Gene expression values are shown on a linear scale. (D) Heatmap summarizing combinatoral 3-gene panels used for multiplex fluorescent in situ hybridization assays to explore the spatial distribution of 10 excitatory clusters. Gene combinations for each cluster are indicated by colored boxes on the heatmap. (E) Representative inverted images of DAPIstained cortical columns spanning layers 1-6 for each marker gene panel. Red dots depict the locations of cells positive for the specific marker gene combinations for each cluster. Marker gene combinations are listed at the top of each image. Cluster names along with color coded cluster-specific bars are beneath each panel. Scale bar, 250 $\mu \mathrm{m}$. Below the DAPI images, a schematic diagram of the spatial distribution (i.e. the laminar extent) of each cluster examined. The schematic is based on the observed positions of labeled cells across $n=3-4$ sections per cell type and $n=2-3$ donors per cell type. Bar plots below summarize counts of the percentage of labeled cells per layer, expressed as a fraction of the total number of labeled cells for each type. Bars are color coded to represent different cortical layers using the scheme shown in (A). The cluster represented by each bar is indicated by the colored bar at the bottom of the plot. Cell 
bioRxiv preprint doi: https://doi.org/10.1101/384826: this version posted August 5, 2018. The copyright holder for this preprint (which was not certified by peer review) is the author/funder, who has granted bioRxiv a license to display the preprint in perpetuity. It is made available under aCC-BY-NC-ND 4.0 International license.

A

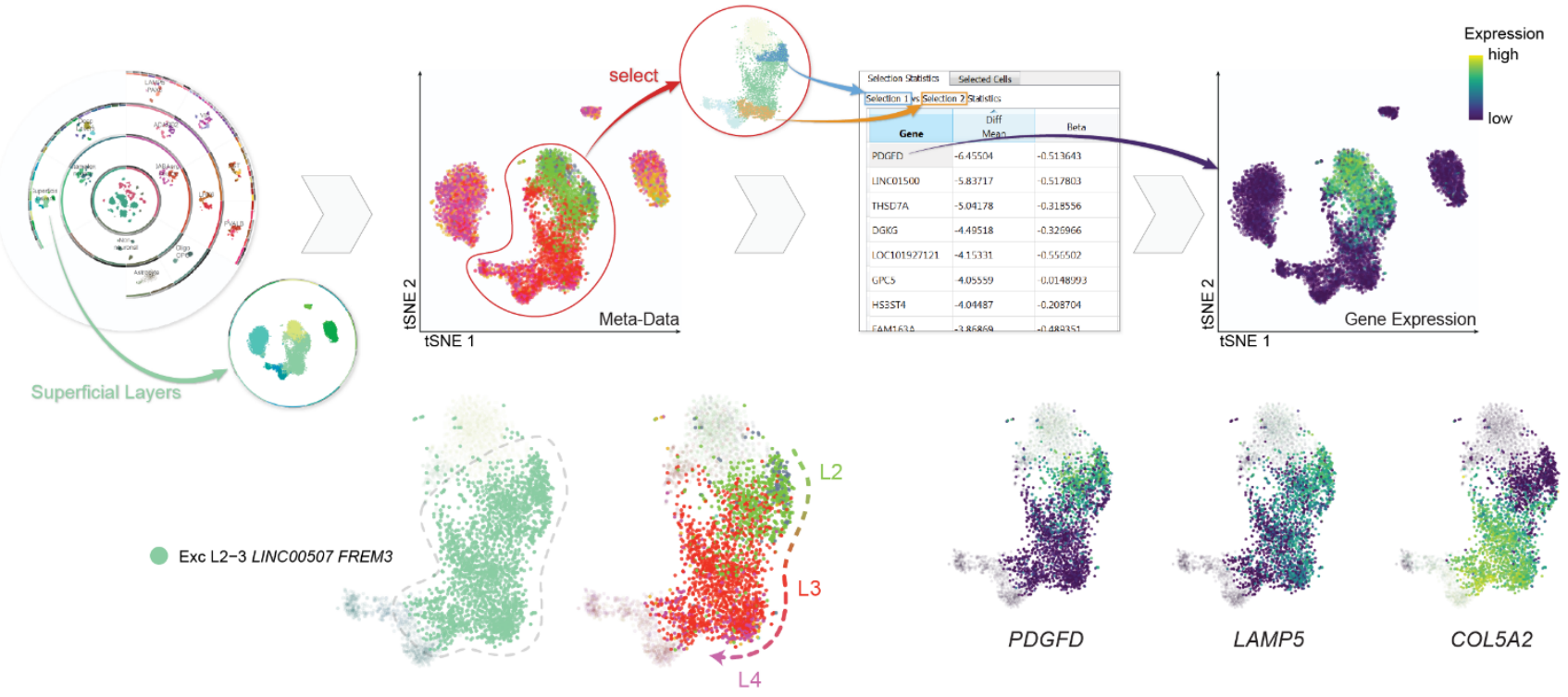

B

Gradient expression within Exc L2-3 LINC00507 FREM3

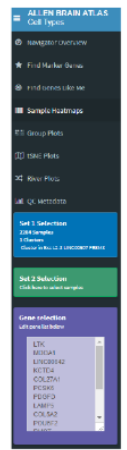

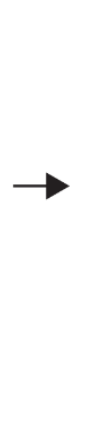

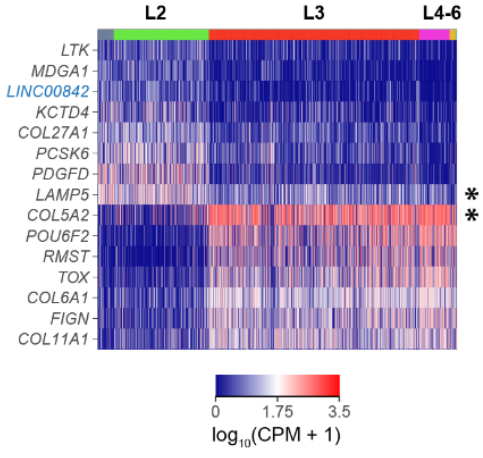

C

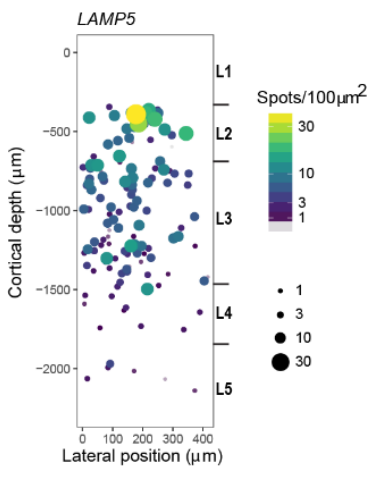

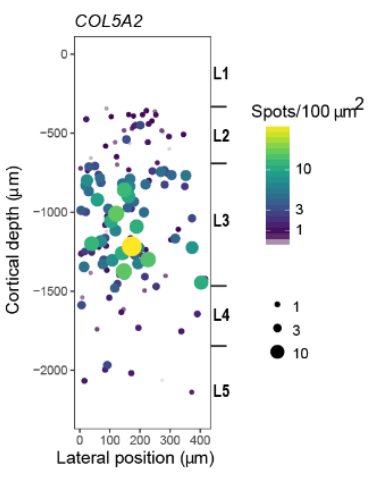


Figure 3. Gene expression heterogeneity within the Exc L2-3 LINC00507 FREM3 cell type. (A, B) Transcriptomics data visualization tools for exploring gene expression gradients in human cortical neurons. (A) Cytosplore MTG Viewer. Top panels, left to right: the hierarchy viewer shows an overview of the t-SNE map of all clusters. Zooming in allows for visualization and selection of superficial layer excitatory neurons on the t-SNE map. Overlaying layer metadata on the t-SNE map shows that nuclei within the EXC L2-3 LINC00507 FREM3 cell type are sorted by cortical layer. Differential expression analysis, computed by selecting nuclei on opposite ends of the cluster, reveals gene expression gradients organized along the layer structure of the cluster. Bottom panels, left to right: t-SNE map showing the EXC L2-3 LINC00507 FREM3 cluster outlined by dashed gray line. Overlaying layer metadata on the cluster highlights its layer structure. Examples of genes that exhibit expression heterogeneity across the layer structure of the cluster are shown to the right. (B) RNA-Seq Data Navigator. Selection of the sample heatmaps option in the browser allows for visualization of gene expression patterns in the EXC L2-3 LINC00507 FREM3 cluster. Each row in the heatmap represents a gene (blue, non-coding), and nuclei in the cluster are ordered by layer (colored bar at the top of the heatmap). The selected genes illustrate opposing gene expression gradients across the layer structure of the cluster. Genes marked with an asterisk were included in the validation experiments in (C). (C) Single molecule fluorescent in situ hybridization (smFISH) validation of gene expression heterogeneity. Panels show quantification of LAMP5 (left) and COL5A2 (right) expression in cells located in layers 2-3. Each circle represents a cell, the size of each circle is proportional to the number of smFISH spots per cell, and circles are colorcoded per the scale shown to the right of each panel. Consistent with the RNA-seq data shown in panels $\mathrm{A}$ and $\mathrm{B}, \mathrm{smFISH}$ analysis demonstrates that these genes exhibit opposing expression gradients across cortical layers 2 and 3 . 
bioRxiv preprint doi: https://doi.org/10.1101/384826; this version posted August 5, 2018. The copyright holder for this preprint (which was not certified by peer review) is the author/funder, who has granted bioRxiv a license to display the preprint in perpetuity. It is made available under aCC-BY-NC-ND 4.0 International license.

A

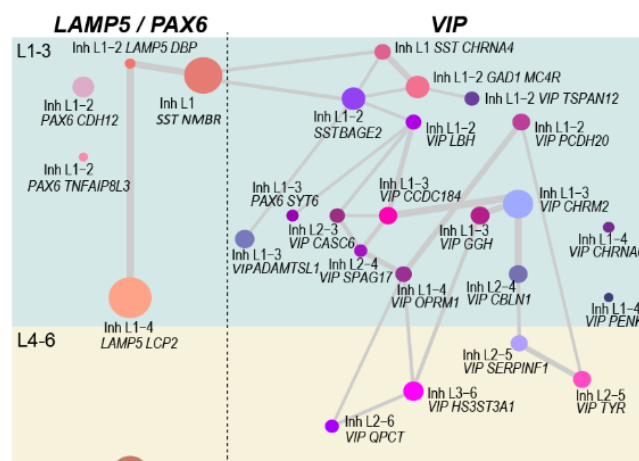

C

E

L1

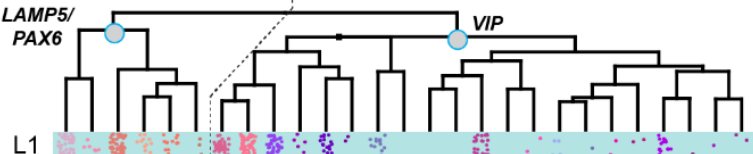

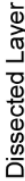

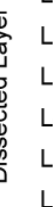

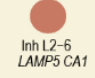

B

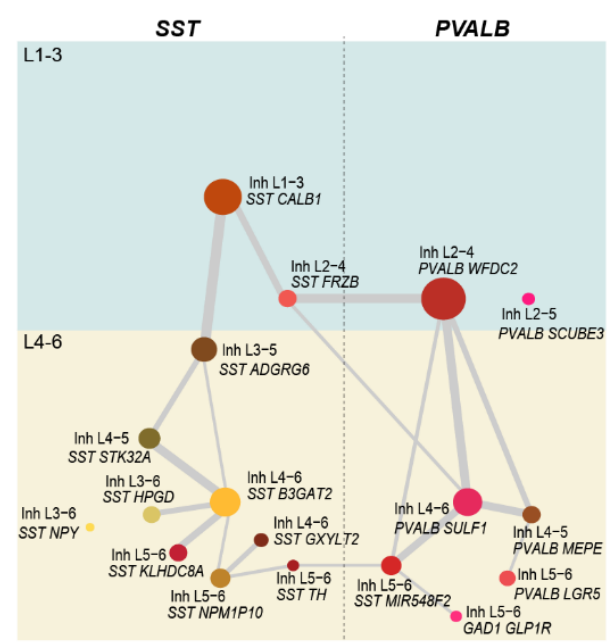

D

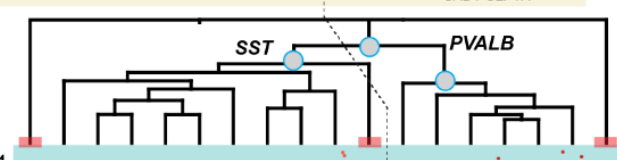

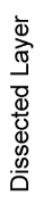
L1

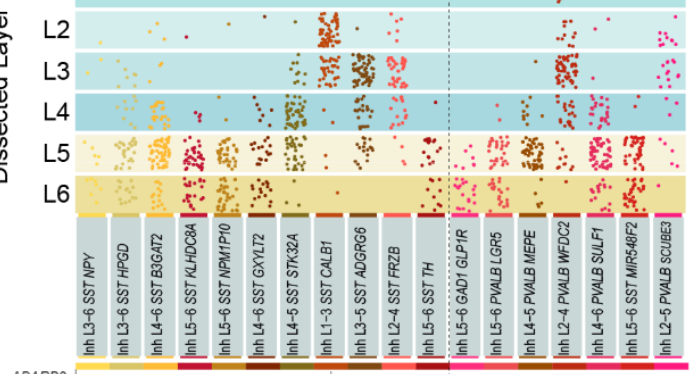

F
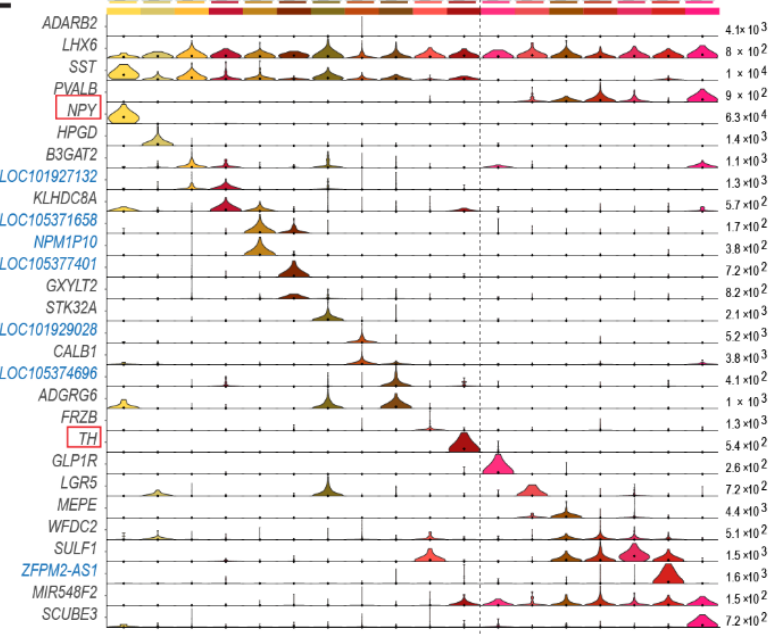

H

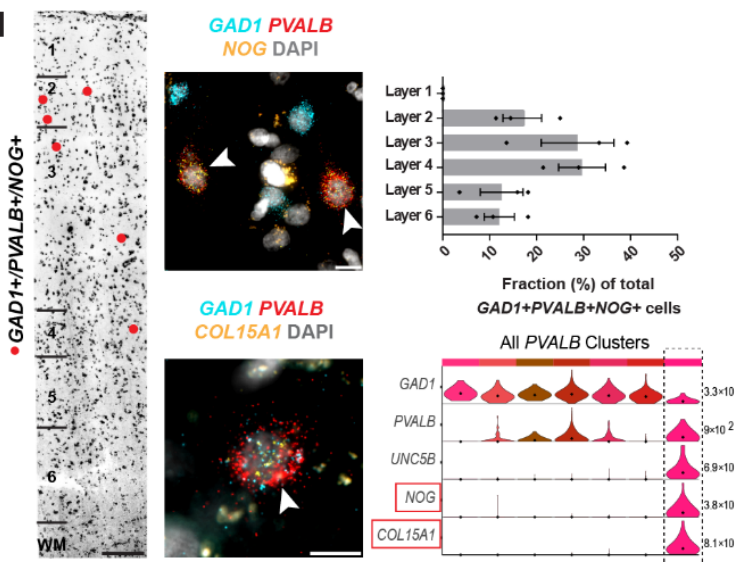

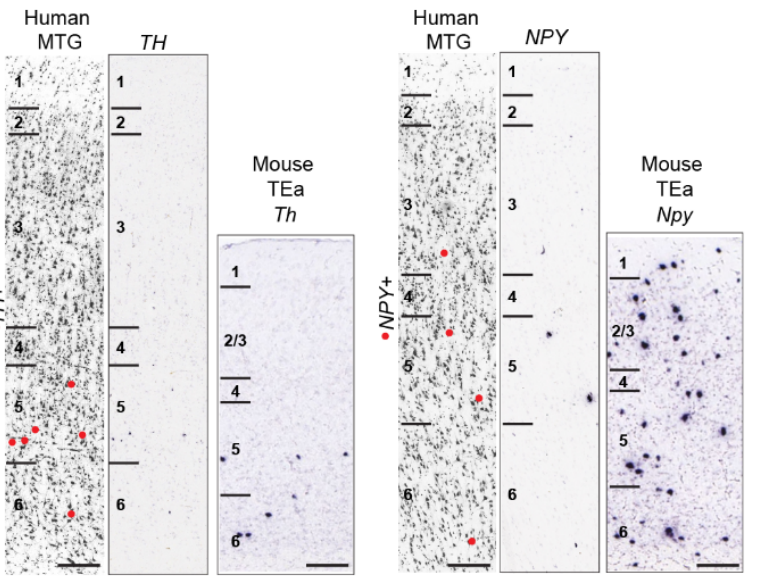


Figure 4. Inhibitory neuron diversity and marker gene expression. (A, B) Constellation diagrams for LAMP5/PAX6 and VIP (A) and SST/PVALB (B) subclasses. The number of core cells within each cluster is represented by disc area and the number of intermediate cells by weighted lines. (C, D) Dendrograms illustrate gene expression similarity between cell types. Below each dendrogram, the spatial distribution of each type is shown. Each dot represents a single nucleus derived from a layer-specific dissection. Red bars at the base of the dendrogram in (D) indicate clusters examined using in situ hybridization (ISH) in (G-H). (E, F) Violin plots of marker gene expression distributions across clusters. Rows are genes (blue, non-coding transcripts), black dots in each violin represent median gene expression within clusters, and the maximum expression value for each gene is shown on the right-hand side of each row. Gene expression values are shown on a linear scale. Genes shown in $(G)$ are outlined by red boxes in (F). (G) Chromogenic single gene ISH for TH (left), a marker of Inh L5-6 SST TH, and NPY (right), a marker of Inh L3-6 SST NPY, from the Allen Human Brain Atlas. Left columns show grayscale images of the Nissl stained section nearest the ISH stained section shown in the right panel for each gene. Red dots overlaid on the Nissl section show the laminar positions of cells positive for the gene assayed by ISH. Chromogenic ISH for Th and Npy in mouse temporal 804 association cortex (TEa) from the Allen Mouse Brain Atlas are to the right of the human ISH images. Scale bars: human $(250 \mu \mathrm{m})$, mouse $(100 \mu \mathrm{m})$. (H) RNAscope mutiplex fluorescent ISH for markers of putative chandelier cell cluster Inh L2-5 PVALB SCUBE3. Left panel representative inverted DAPI-stained cortical column with red dots marking the position of cells positive for the genes GAD1, PVALB, and NOG (scale bar, 250um). Middle - images of cells positive for GAD1, PVALB, and the specific marker genes NOG (top, scale bar 10 $\mu \mathrm{m}$ ) and COL15A1 (bottom, scale bar 10um). White arrows mark triple positive cells. Right - bar plot summarizes counts of GAD1+, PVALB+, NOG+ cells across layers (expressed as percentage of total triple positive cells). Bars show the mean, error bars represent the standard error of the mean (SEM), and dots represent data points for individual specimens ( $n=3$ subjects). Violin plot shows gene expression distributions across clusters in the PVALB subclass for the chandelier cell marker UNC5B and the Inh L2-5 PVALB SCUBE3 cluster markers NOG and COL15A1. 
bioRxiv preprint doi: https://doi.org/10.1101/384826; this version posted Auqust 5, 2018. The copyright holder for this preprint (which was not certified by peer review) is the author/funder, who has granted bioRxiv a license to display the preprint in perpetuity. It is made available under aCC-BY-NC-ND 4.0 International license.

A

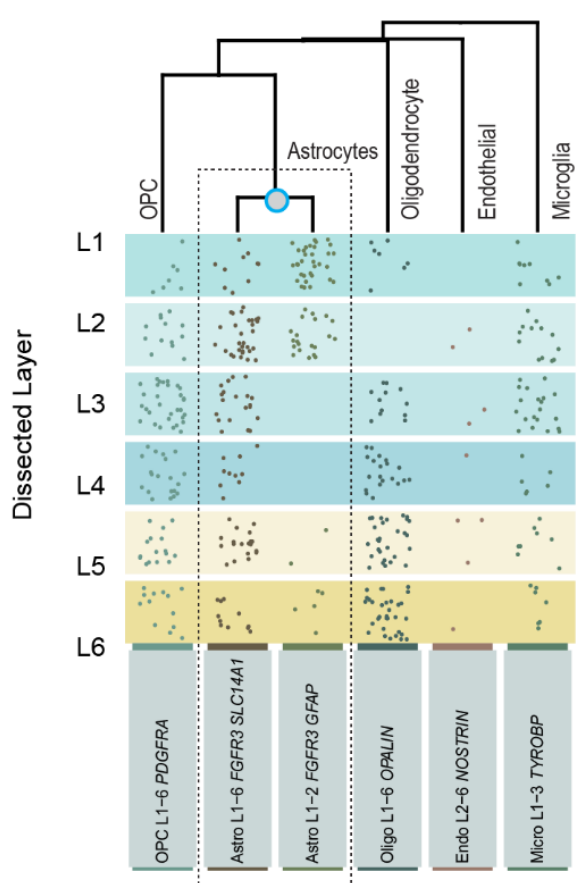

B
C
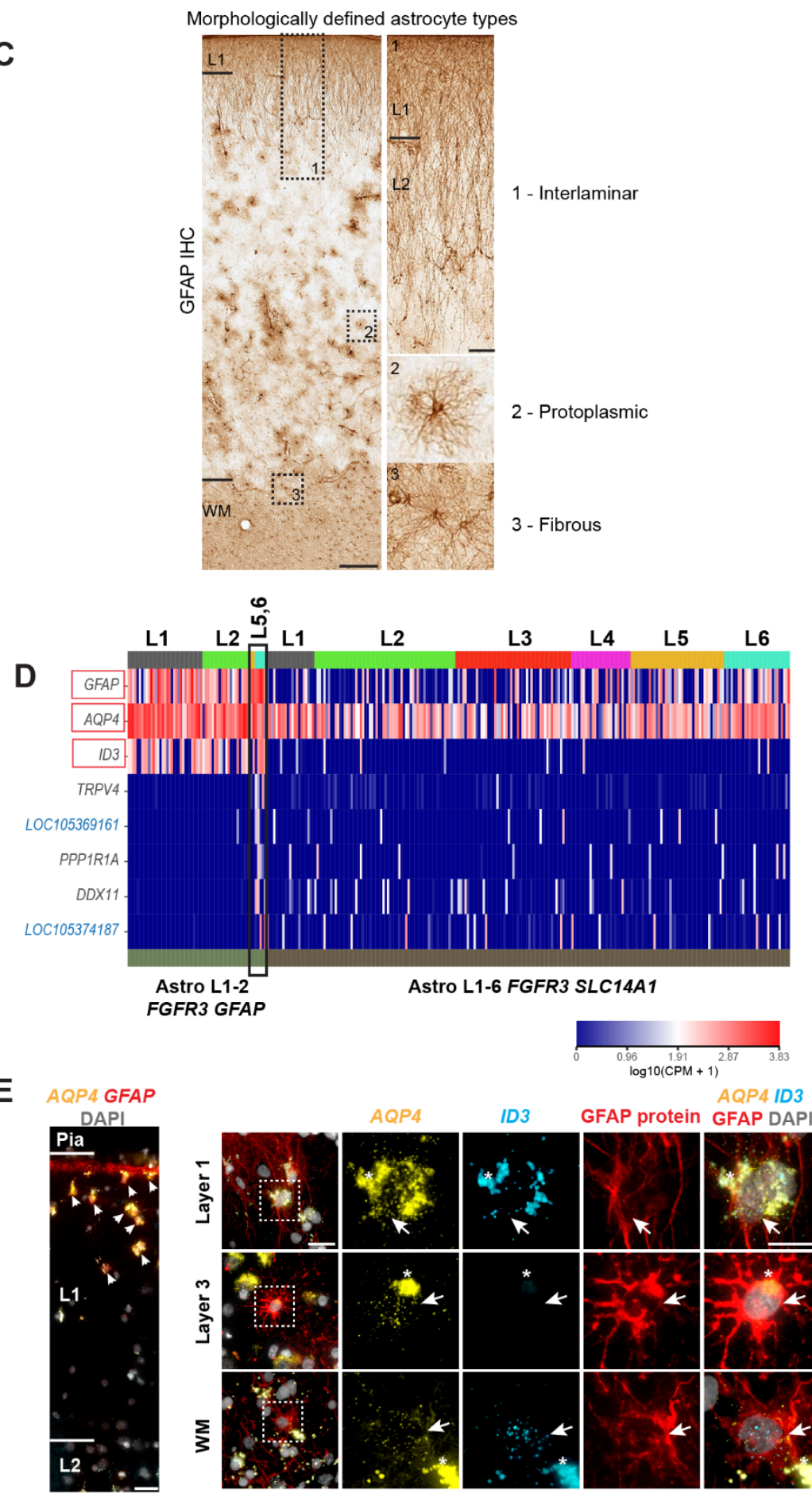

AQP4
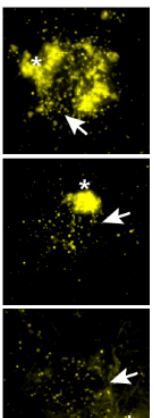

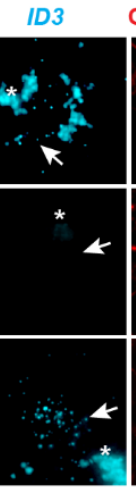

GFAP prote

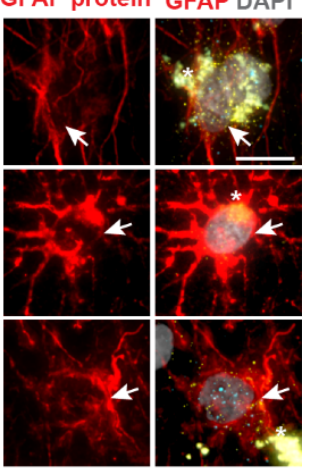


Figure 5. Non-neuronal cell type diversity and marker gene expression. (A) Dendrogram illustrating overall gene expression similarity between non-neuronal cell types, with the spatial distribution of types shown beneath the dendrogram. Each dot represents a single nucleus from a layer-specific dissection. (B) Violin plots show expression distributions of marker genes across clusters. Each row represents a gene (blue, non-coding), black dots represent median gene expression within clusters, and the maximum expression value for each gene is shown on the right-hand side of each row. Gene expression values are shown on a linear scale. (C) Immunohistochemistry (IHC) for GFAP in human MTG illustrates the features of morphologically-defined astrocyte types. Black boxes on the left panel indicate regions shown at higher magnification on the right. Scale bars: low mag $(250 \mu \mathrm{m})$, high mag $(50 \mu \mathrm{m})$. (D) Heatmap illustrating marker gene expression in the Astro L1-2 FGFR3 GFAP and Astro L1-6 FGFR3 $S L C 14 A 1$ clusters. Each row is a gene, each column a single nucleus, and the heatmap is ordered per the layers that nuclei were dissected from. A minority of nuclei in the Astro L1-2 FGFR3 GFAP cluster came from deep layers (black box on heatmap) and express marker genes distinct from the other nuclei in the cluster. Red boxes in $(B, D)$ are genes examined in (E). (E) RNAscope multiplex fluorescent in situ hybridization (FISH) for astrocyte markers. Left expression of $A Q P 4$ and GFAP in layer 1 (scale bar, 25 $\mu \mathrm{m}$ ). Cells expressing high levels of AQP4 and GFAP, consistent with the Astro L1-2 FGFR3 GFAP cluster, are localized to the top half of layer 1 (white arrowheads). Right - FISH for AQP4 and ID3 combined with GFAP immunohistochemistry. White box indicates area shown at higher magnification to the right. Scale bars: low mag $(25 \mu \mathrm{m})$, high mag $(15 \mu \mathrm{m})$. Asterisks mark lipofuscin autofluoresence. Top row: AQP4 expressing cells in layer 1 coexpress ID3 and have long, GFAP-labeled processes that span layer 1. Middle row: protoplasmic astrocyte located in layer 3 lacks expression of ID3, consistent with the Astro L1-6 FGFR3 SLC14A1 type. Bottom row: fibrous astrocyte at the white matter (WM)/layer 6 boundary triple positive for AQP4, ID3, and GFAP protein. 
bioRxiv preprint doi: httos://doi ora/10 1101/384826: this version posted Auqust 5 , 2018. The copvriaht holder for this preprint (which was not certified by peer review) is the author/funder, who has granted bioRxiv a license to display the preprint in perpetuity. It is made available under aCC-BY-NC-ND 4.0 International license.
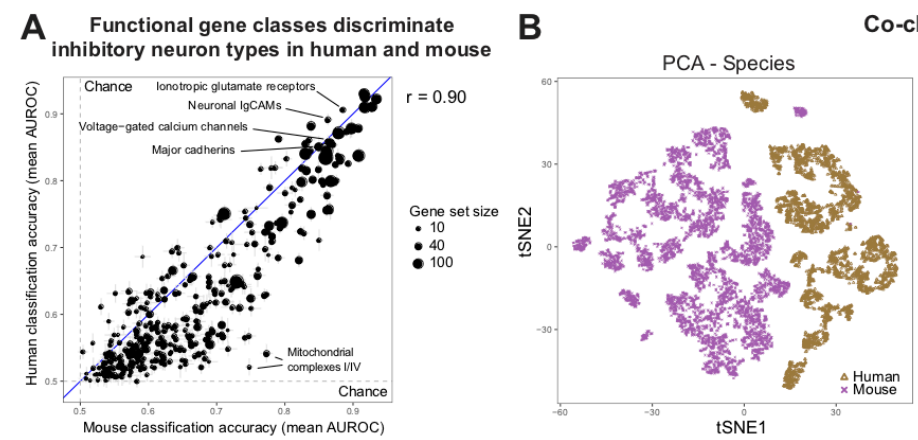

Co-clustering human and mouse inhibitory neurons
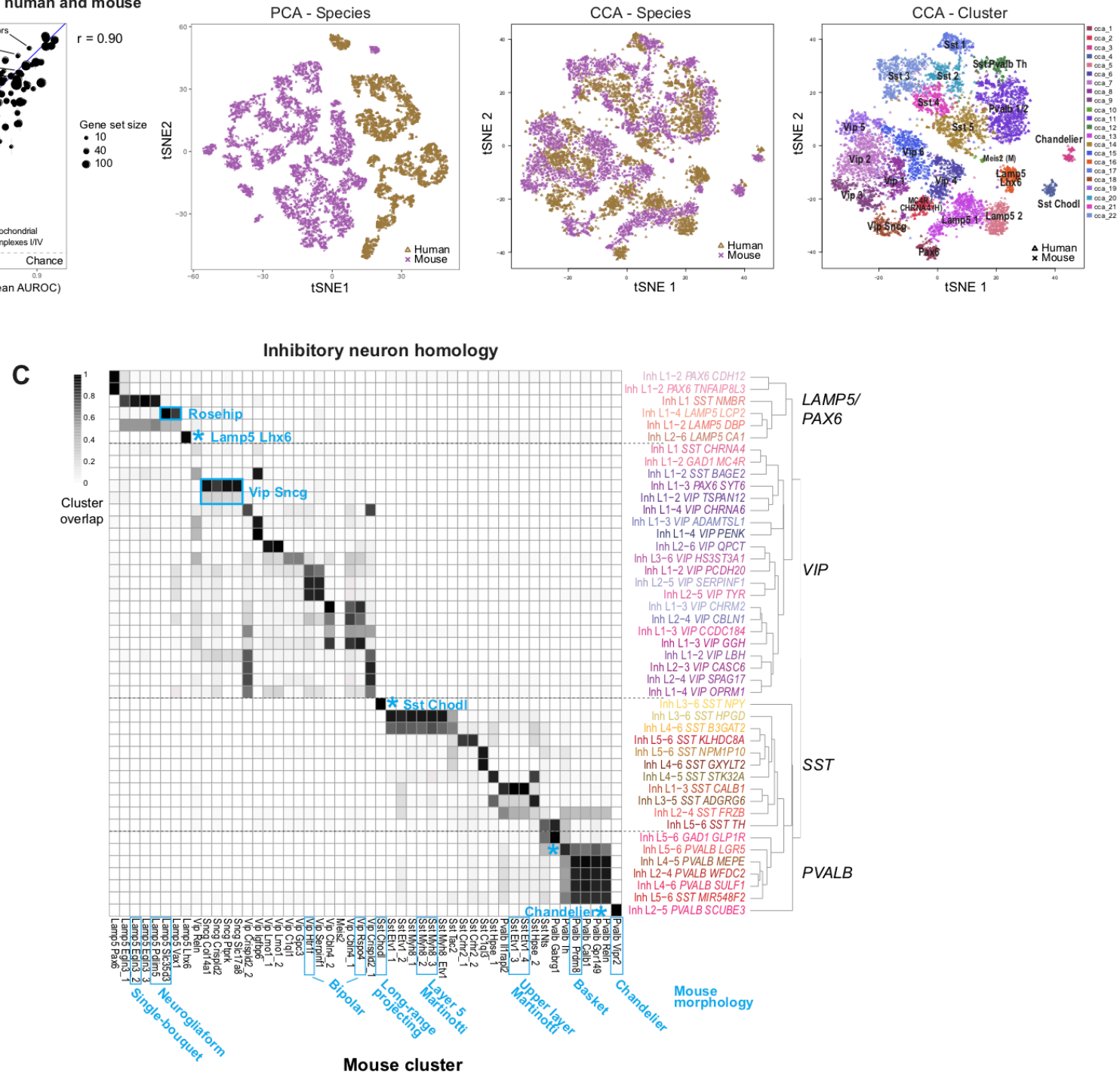

D

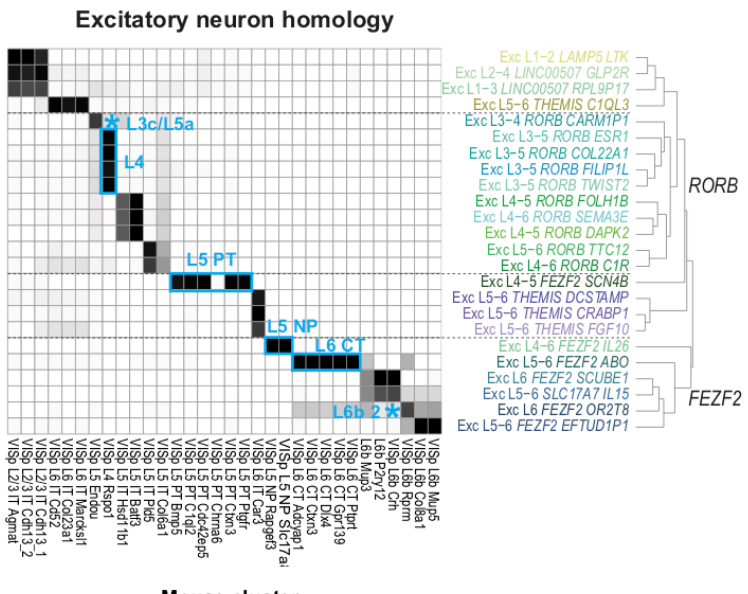

$\mathbf{E}$

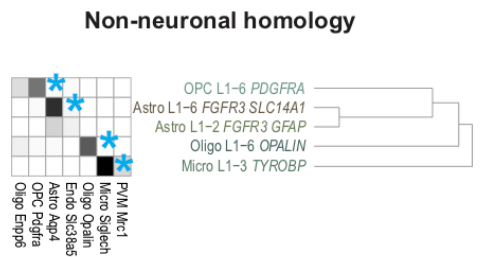

Mouse cluster

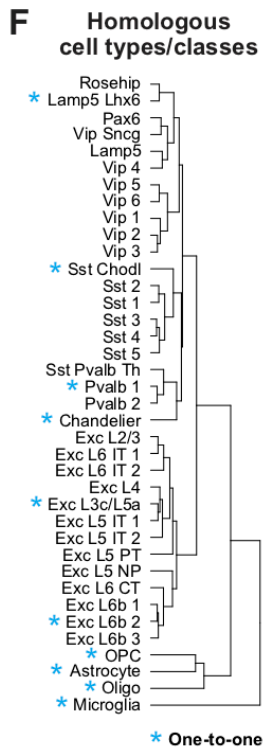


844 Figure 6. Evolutionary conservation of cell types between human and mouse. (A) Inhibitory neurons can be assigned to cell types based on expression patterns of functional gene families ( $n=5$ to 261 genes). Classification performance (average MetaNeighbor AUROC score across clusters) varies among functional classes of genes and is highly correlated $(r=$ 0.90 ) between human and mouse. Error bars correspond to the standard deviation of average AUROC scores across ten sub-sampled iterations. (B) Human (gold) and mouse (purple) inhibitory neurons were aligned with principal components analysis (PCA; left) and canonical correlation analysis (CCA; middle), and the first 30 basis vectors were represented using t-SNE. Right: CCA clusters were identified by the Louvain algorithm using 30 nearest neighbors and annotated based on cluster labels from this study and mouse. Clusters labeled with $(M)$ or $(H)$ contain only mouse cells or human nuclei, respectively. (C-E) Human and mouse cell type homologies for inhibitory neurons (C), excitatory neurons (D), and non-neuronal cells (E) were predicted based on shared CCA cluster membership. Greyscale indicates, for each pair of human (rows) and mouse (columns) clusters, the minimum proportion of human nuclei or mouse cells that co-cluster using CCA. Note that rows and columns need not sum to one because clusters can partially overlap. One-to-one matches are indicated by an asterisk. Known morphologies are indicated for mouse inhibitory types and known projection targets are given for excitatory types (IT - intratelencephalic, PT - pyramidal tract/sub-cortical, NP - nearprojecting, CT - corticothalamic). Note that human endothelial nuclei could not be aligned by CCA and were excluded from the analysis. (F) Hierarchical taxonomy of 34 neuronal and 4 nonneuronal homologous cell types and cell classes, including 10 cell types that match one-to-one between human and mouse. 
bioRxiv preprint doi: https://doi. org/10.1101/384826; this version posted August 5 , 2018. The copyright holder for this preprint (which was not certified by peer review) is the author/funder, who has granted bioRxiv a license to display the preprint in perpetuity. It is made available under aCC-BY-NC-ND 4.0 International license.

A
Human
Mouse
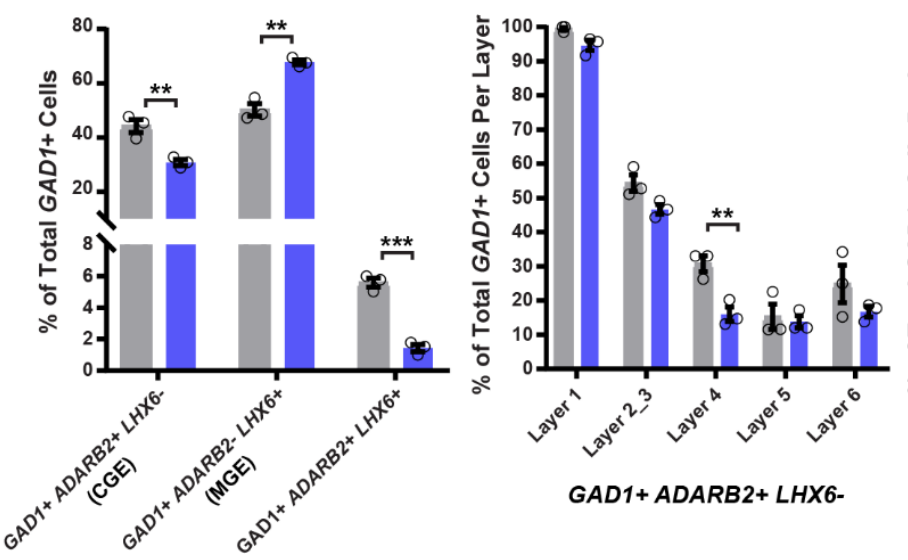

GAD1+ ADARB2+ LHX6-
CGE

LAMP5/PAX6, VIP
B
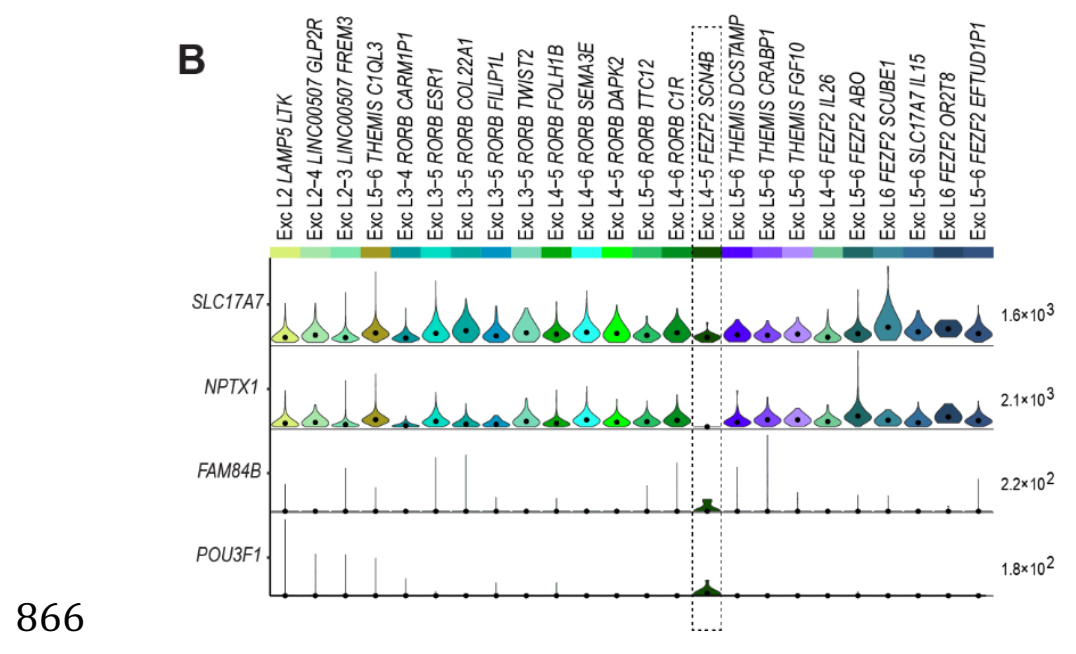

MGE

PVALB, SST

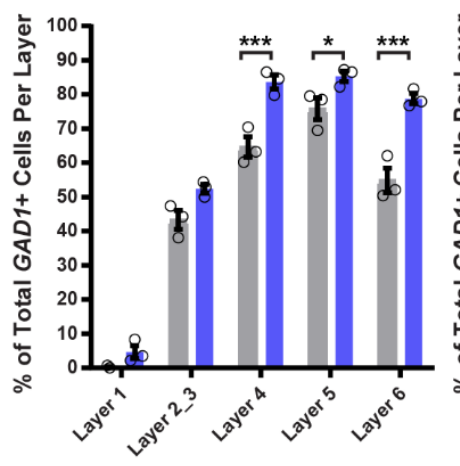

GAD1+ ADARB2- LHX6+
Inh L2-6 LAMP5 CA1 (Human)

Lamp5 Lhx6 (Mouse)
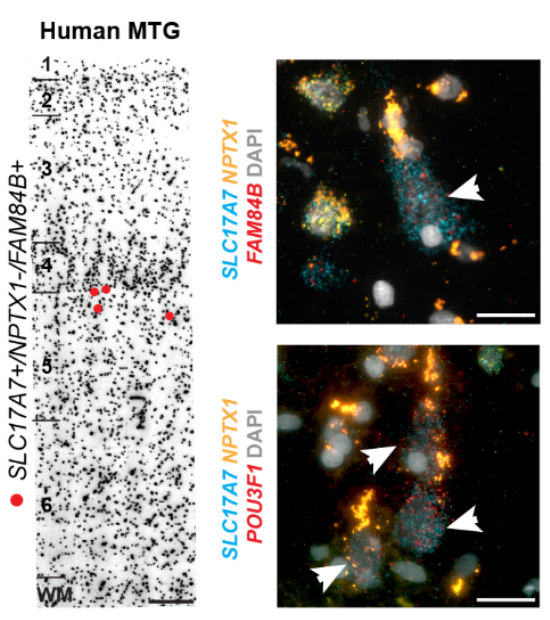

Mouse TEa Fam84b ISH

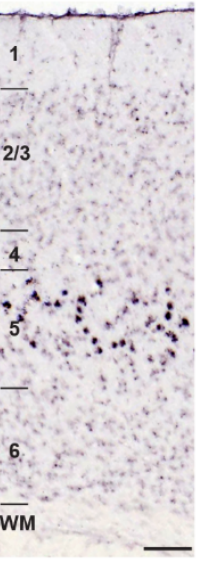


867 Figure 7. Frequency differences in cell classes and types between human and mouse. (A) Quantification of broad interneuron classes in human MTG and mouse temporal association area (TEa) based on counts of cells labeled using RNAscope multiplex fluorescent in situ hybridization (FISH). Sections were labeled with the gene panel GAD1/Gad1, LHX6/Lhx6, and $A D A R B 2 / A d a r b 2$ (human/mouse). Bar plots from left to right: (1) Percentage of each major cell class of total GAD1+ cells. (2) Percentage of GAD1+/ADARB2+/LHX6- cells of total GAD1+ cells per layer, representing LAMP5/PAX6, and VIP types. (3) Percentage of GAD1+/ADARB2$/ L H X 6+$ cells of total GAD1+ cells per layer, representing all PVALB and SST types. (4) Percentage of $G A D 1+/ A D A R B 2+/ L H X 6+$ cells of total $G A D 1+$ cells per layer, representing the Inh L2-6 LAMP5 CA1 type (human) or Lamp5 Lhx6 type (mouse). Bars represent the mean, error bars the standard error of the mean, and circles show individual data points for each specimen ( $n=3$ specimens for both human and mouse; t-test with Holm-Sidak correction for multiple comparisons, $\left.{ }^{*} p<0.05^{* *} p<0.01,{ }^{* * *} p<0.001\right)$. (B) Left to right: violin plot showing expression of specific markers of the putative pyramidal tract (PT) EXC L4-5 FEZF2 SCN4B

881 cluster (black box) and NPTX1, a gene expressed by all non-PT excitatory neurons. Each row

882 represents a gene, the black dots in each violin represent median gene expression within

883 clusters, and the maximum expression value for each gene is shown on the right-hand side of

884 each row. Gene expression values are shown on a linear scale. A representative inverted

885 DAPI-stained cortical column (scale bar, $200 \mu \mathrm{m}$ ) with red dots marking the position of cells 886 positive for the genes SLC17A7 and FAM84B and negative for NPTX1 illustrates the relative abundance of the EXC L4-5 FEZF2 SCN4B type in human MTG. Representative examples of RNAscope multiplex FISH stained sections from human MTG showing FAM84B (top, white arrows, scale bar, $25 \mu \mathrm{m}$ ) and POU3F1-expressing cells (bottom, white arrows, scale bar, $25 \mu \mathrm{m}$ ). Expression of Fam84b in mouse TEa (scale bar, $75 \mu \mathrm{m}$ ) is shown in the adjacent panel. 
bioRxiv preprint doi: https://doi. org/10.1101/384826: this version posted August 5 , 2018. The copyright holder for this preprint (which was not certified by peer review) is the author/funder, who has granted bioRxiv a license to display the preprint in perpetuity. It is made available under aCC-BY-NC-ND 4.0 International license.
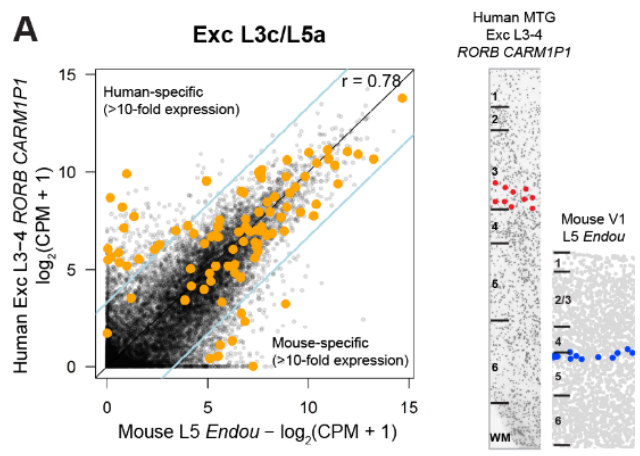

\section{C}

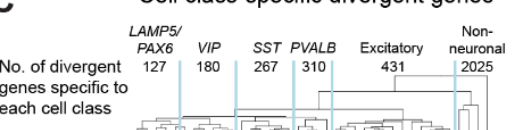

B

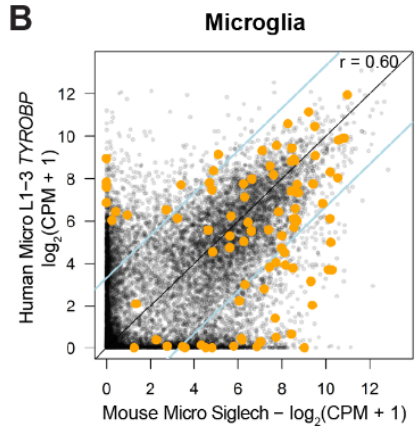

$\mathbf{F}$

Top 20 most divergent functional gene classes

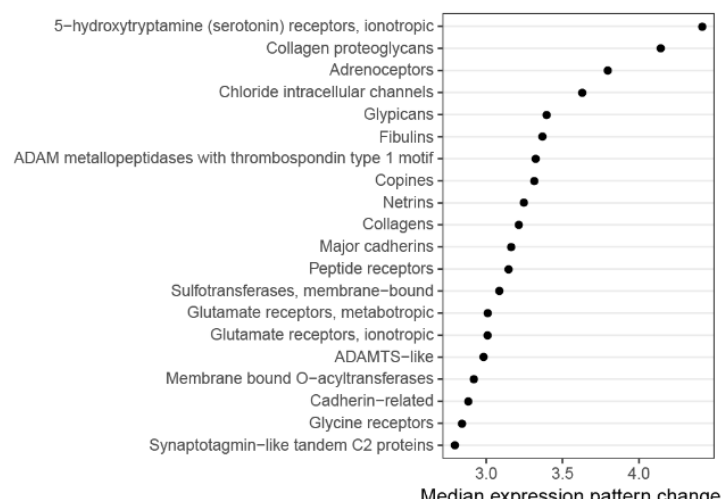

G Extracellular matrix (collagens)

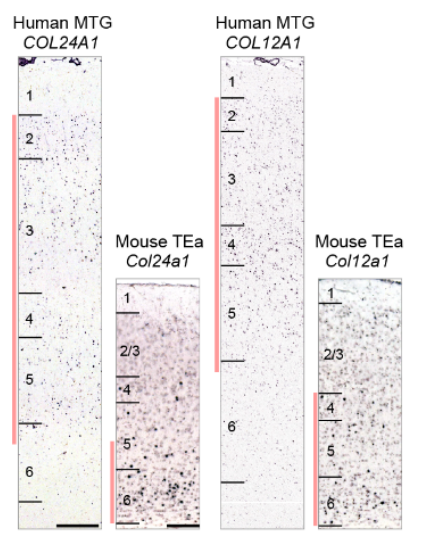

D Expression divergence

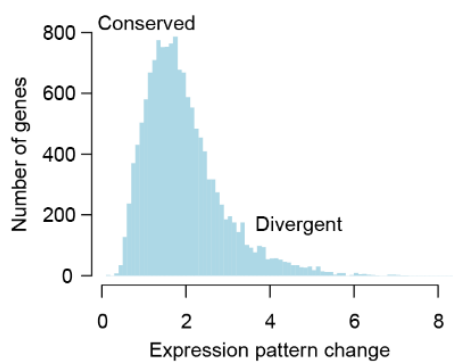

E

Cell type specific genes have more divergent expression

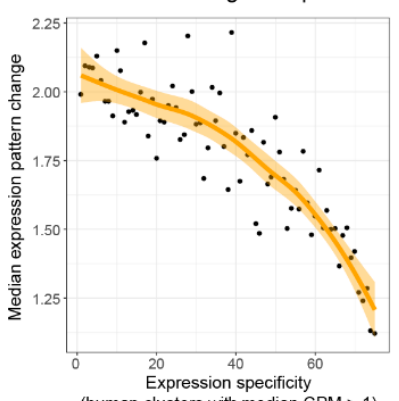

Glutamate receptors

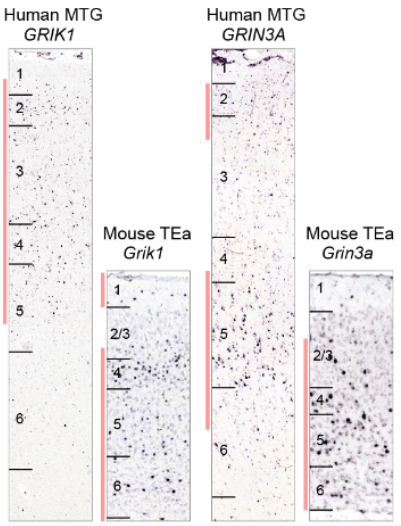


892 Figure 8. Divergent cell type expression between human and mouse. (A) Left: Comparison of expression levels of 14,414 orthologous genes between human and mouse for the most

894 highly conserved one-to-one homologous type, Exc L3c/L5a. Genes outside of the blue lines have highly divergent expression (>10-fold change) between human and mouse. Approximately 100 genes (orange dots) are relatively specific markers in human and/or mouse. Right: ISH validation of layer distributions in human MTG and mouse primary visual cortex (data from Tasic et al., 2017). Cells are labeled based on expression of cluster marker genes in human (RORB+/CNR1-/PRSS12+) and mouse (Scnn1a+/Hsd11b1+). (B) Comparison of expression between human and mouse microglia, the least conserved homologous type. (C) Patterns of expression divergence between human and mouse for 8222 genes (57\% of orthologous genes) with at least 10 -fold expression change in one or more homologous cell types. Genes were hierarchically clustered and groups of genes that have similar patterns of expression divergence are labeled by the affected cell class. Top row: number of genes with expression divergence restricted to each broad class of cell types. (D) For each gene, the expression pattern change was quantified by the beta score (see Methods) of the absolute log fold change in expression between human and mouse. Genes with divergent patterns have large expression changes among a subset of homologous cell types. Genes with conserved patterns have similar expression levels in human and mouse or have a similar expression level change in all types. Pattern changes are approximately log-normally distributed, and a minority of genes have highly divergent patterns. (E) Genes expressed in fewer human cell types tended to have greater evolutionary divergence than more ubiquitously expressed genes. A loess curve and standard error was fit to median expression pattern changes across genes binned by numbers of clusters with expression (median CPM > 1). (F) Gene families with the most divergent expression patterns (highest median pattern change) include neurotransmitter receptors, ion channels, and cell adhesion molecules. (G) Genes estimated to have highly divergent expression patterns

918 layers with enriched expression. Scale bars: human $(250 \mu \mathrm{m})$, mouse $(100 \mu \mathrm{m})$. 


\section{Methods}

\section{Post-mortem tissue donors}

921 Males and females 18 - 68 years of age with no known history of neuropsychiatric or neurological conditions ('control' cases) were considered for inclusion in this study (Extended Data Table 1). De-identified postmortem human brain tissue was collected after obtaining permission from decedent next-of-kin. The Western Institutional Review Board (WIRB) reviewed the use of de-identified postmortem brain tissue for research purposes and determined that, in accordance with federal regulation 45 CFR 46 and associated guidance, the use of and generation of data from de-identified specimens from deceased individuals did not constitute human subjects research requiring IRB review. Postmortem tissue collection was performed in accordance with the provisions of the Uniform Anatomical Gift Act described in Health and Safety Code $\S \S 7150$, et seq., and other applicable state and federal laws and regulations. Routine serological screening for infectious disease (HIV, Hepatitis B, and Hepatitis C) was conducted using donor blood samples and only donors negative for all three tests were considered for inclusion in the study. Tissue RNA quality was assessed using an Agilent Bioanalyzer-generated RNA Integrity Number (RIN) and Agilent Bioanalyzer electropherograms for $18 \mathrm{~S} / 28 \mathrm{~S}$ ratios. Specimens with RIN values $\geq 7.0$ were considered for inclusion in the study (Extended Data Table 1).

\section{Processing of whole brain postmortem specimens}

938 Whole postmortem brain specimens were transported to the Allen Institute on ice. Standard processing of whole brain specimens involved bisecting the brain through the midline and embedding of individual hemispheres in Cavex Impressional Alginate for slabbing. Coronal brain slabs were cut at $1 \mathrm{~cm}$ intervals through each hemisphere and individual slabs were frozen in a slurry of dry ice and isopentane. Slabs were then vacuum sealed and stored at $-80^{\circ} \mathrm{C}$ until the time of further use.

Middle temporal gyrus (MTG) was identified on and removed from frozen slabs of interest, and subdivided into smaller blocks for further sectioning. Individual tissue blocks were processed by thawing in PBS supplemented with 10mM DL-Dithiothreitol (DTT, Sigma Aldrich), mounting on a vibratome (Leica), and sectioning at $500 \mu \mathrm{m}$ in the coronal plane. Sections were placed in fluorescent Nissl staining solution (Neurotrace 500/525, ThermoFisher Scientific) prepared in PBS with 10mM DTT and 0.5\% RNasin Plus RNase inhibitor (Promega) and stained for 5 min on ice. After staining, sections were visualized on a fluorescence dissecting microscope (Leica) and cortical layers were individually microdissected using a needle blade micro-knife (Fine

953 Science Tools).

\section{Neurosurgical tissue donors}

955 Tissue procurement from neurosurgical donors was performed outside of the supervision of the authority of the IRB of each participating hospital. A hospital-appointed case coordinator obtained informed consent from donors prior to surgery. Tissue specimens were de-identified prior to receipt by Allen Institute personnel. The specimens collected for this study were apparently non-pathological tissues removed during the normal course of surgery to access underlying pathological tissues. Tissue specimens collected were determined to be nonessential for diagnostic purposes by medical staff and would have otherwise been discarded. 


\section{Processing of neurosurgical tissue samples}

964 Neurosurgical tissue was transported to the Allen Institute in chilled, oxygenated artificial cerebrospinal fluid (ACSF) consisting of the following: $0.5 \mathrm{mM}$ calcium chloride (dehydrate), 25 mM D-glucose, $20 \mathrm{mM}$ HEPES, $10 \mathrm{mM}$ magnesium sulfate, $1.2 \mathrm{mM}$ sodium phosphate monobasic monohydrate, $92 \mathrm{mM} \mathrm{N}$-methyl-d-glucamine chloride (NMDG-Cl), $2.5 \mathrm{mM}$ potassium chloride, $30 \mathrm{mM}$ sodium bicarbonate, $5 \mathrm{mM}$ sodium L-ascorbate, $3 \mathrm{mM}$ sodium pyruvate, and 2 $\mathrm{mM}$ thiourea. The osmolality of the solution was $295-305 \mathrm{mOsm} / \mathrm{kg}$ and the $\mathrm{pH}$ was 7.3 . Slices were prepared using a Compresstome VF-200 or VF-300 vibratome (Precisionary Instruments). After sectioning, slices were recovered in ACSF containing $2 \mathrm{mM}$ calcium chloride (dehydrate), $25 \mathrm{mM}$ D-glucose, $20 \mathrm{mM}$ HEPES, $2 \mathrm{mM}$ magnesium sulfate, $1.2 \mathrm{mM}$ sodium phosphate monobasic monohydrate, $2.5 \mathrm{mM}$ potassium chloride, $30 \mathrm{mM}$ sodium bicarbonate, $92 \mathrm{mM}$ sodium chloride, $5 \mathrm{mM}$ sodium L-ascorbate, $3 \mathrm{mM}$ sodium pyruvate, and $2 \mathrm{mM}$ thiourea at room temperature for at least 1 hour. After the recovery period, slices were transferred to RNase-free microcentrifuge tubes, snap frozen, and stored at $-80^{\circ} \mathrm{C}$ until the time of use. Microdissection of cortical layers was carried out on tissue slices that were thawed and stained as described above for postmortem tissue.

\section{Nucleus sampling plan}

We estimated that 16 cells were required to reliably discriminate two closely related Sst+ interneuron types reported by ${ }^{27}$. Monte Carlo simulations were used to estimate the sampling depth $N$ needed to be $95 \%$ confident that at least 16 nuclei of frequency $f$ have been selected from the population. Calculating $N$ for a range of $f$ revealed a simple linear approximation: $N=$ $28 / f$. Subtypes of mouse cortical layer 5 projection neurons can be rarer than $1 \%$ of the population ${ }^{72}$, so we targeted neuron types as rare as $0.2 \%$ of all cortical neurons. We initially sampled 14,000 neuronal nuclei distributed across cortical layers relative to the proportion of neurons reported in each layer ${ }^{36}$. We sampled approximately 1000 additional neuronal nuclei from layers with increased diversity observed based on RNA-seq data. We also targeted 1500 $(10 \%)$ non-neuronal (NeuN-) nuclei and obtained approximately 1000 nuclei that passed QC, and we expected to capture types as rare as $3 \%$ of the non-neuronal population.

\section{Nucleus isolation and sorting}

992 Microdissected tissue pieces were placed in into nuclei isolation medium containing $10 \mathrm{mM}$ Tris $\mathrm{pH} 8.0$ (Ambion), $250 \mathrm{mM}$ sucrose, $25 \mathrm{mM} \mathrm{KCl}$ (Ambion), $5 \mathrm{mM} \mathrm{MgCl}$ (Ambion) $0.1 \%$ Triton-X 100 (Sigma Aldrich), 1\% RNasin Plus, 1X protease inhibitor (Promega), and 0.1mM DTT in $1 \mathrm{ml}$ dounce homogenizer (Wheaton). Tissue was homogenized using 10 strokes of the loose dounce pestle followed by 10 strokes of the tight pestle and the resulting homogenate was passed through $30 \mu \mathrm{m}$ cell strainer (Miltenyi Biotech) and centrifuged at $900 \mathrm{xg}$ for $10 \mathrm{~min}$ to pellet nuclei. Nuclei were resuspended in buffer containing 1X PBS (Ambion), 0.8\% nucleasefree BSA (Omni-Pur, EMD Millipore), and 0.5\% RNasin Plus. Mouse anti-NeuN conjugated to PE (EMD Millipore) was added to preparations at a dilution of 1:500 and samples were incubated for $30 \mathrm{~min}$ at $4^{\circ} \mathrm{C}$. Control samples were incubated with mouse lgG1,k-PE Isotype control (BD Pharmingen). Samples were then centrifuged for $5 \mathrm{~min}$ at $400 \mathrm{xg}$ to pellet nuclei and pellets were resuspended in $1 \mathrm{X}$ PBS, $0.8 \%$ BSA, and 0.5\% RNasin Plus. DAPI (4', 6-diamidino2-phenylindole, ThermoFisher Scientific) was applied to nuclei samples at a concentration of

$1005 \quad 0.1 \mu \mathrm{g} / \mathrm{ml}$.

1006 Single nucleus sorting was carried out on either a BD FACSAria II SORP or BD FACSAria Fusion instrument (BD Biosciences) using a $130 \mu \mathrm{m}$ nozzle. A standard gating strategy was applied to all samples. First, nuclei were gated on their size and scatter properties and then on 
1009 DAPI signal. Doublet discrimination gates were used to exclude nuclei aggregates. Lastly, nuclei were gated on NeuN signal (PE). Ten percent of nuclei were intentionally sorted as NeuN-negative and the remaining $90 \%$ of nuclei were NeuN-positive. Single nuclei were sorted into 8-well strip tubes containing 11.5 $\mu$ l of SMART-seq v4 collection buffer (Takara) supplemented with ERCC MIX1 spike-in synthetic RNAs at a final dilution of 1×10-8 (Ambion). Strip tubes containing sorted nuclei were briefly centrifuged and stored at $-80^{\circ} \mathrm{C}$ until the time of further processing. Index sorting was carried out for most samples to allow properties of nuclei detected during sorting to be connected with the cell type identity revealed by subsequent

1017 snRNA-seq.

\section{RNA-sequencing}

We used the SMART-Seq v4 Ultra Low Input RNA Kit for Sequencing (Takara \#634894) per the manufacturer's instructions for reverse transcription of RNA and subsequent cDNA amplification. Standard controls were processed alongside each batch of experimental samples. Control strips included: 2 wells without cells, 2 wells without cells or ERCCs (i.e. no template controls), and either 4 wells of $10 \mathrm{pg}$ of Human Universal Reference Total RNA (Takara 636538) or 2 wells of 10 pg of Human Universal Reference and 2 wells of 10 pg Control RNA provided in the Clontech kit. cDNA was amplified with 21 PCR cycles after the reverse transcription step. AMPure XP Bead (Beckman Coulter A63881) purification was done using an Agilent Bravo NGS Option A instrument with a bead ratio of $1 \mathrm{x}$, and purified cDNA was eluted in $17 \mu \mathrm{l}$ elution buffer provided by Takara. All samples were quantitated using PicoGreen ${ }^{\circledR}$

1029

1030

1031 (ThermoFisher Scientific) on a Molecular Dynamics M2 SpectraMax instrument. cDNA libraries were examined on either an Agilent Bioanalyzer 2100 using High Sensitivity DNA chips or an Advanced Analytics Fragment Analyzer (96) using the High Sensitivity NGS Fragment Analysis Kit (1bp-6000bp). Purified cDNA was stored in 96 -well plates at $-20^{\circ} \mathrm{C}$ until library preparation.

1034

1035

The NexteraXT DNA Library Preparation (Illumina FC-131-1096) kit with NexteraXT Index Kit

1036

1037 V2 Sets A-D (FC-131-2001, 2002, 2003, or 2004) was used for sequencing library preparation. NexteraXT DNA Library prep was done at either $0.5 \mathrm{x}$ volume manually or $0.4 \mathrm{x}$ volume on the Mantis instrument (Formulatrix). Three different cDNA input amounts were used in generating the libraries: $75 \mathrm{pg}, 100 \mathrm{pg}$, and 125pg. AMPure XP bead purification was done using the Agilent Bravo NGS Option A instrument with a bead ratio of $0.9 x$ and all samples were eluted in $22 \mu l$ of Resuspension Buffer (Illumina). Samples were quantitated using PicoGreen on a Molecular Bynamics M2 SpectraMax instrument. Sequencing libraries were assessed using either an Agilent Bioanalyzer 2100 with High Sensitivity DNA chips or an Advanced Analytics Fragment Analyzer with the High Sensitivity NGS Fragment Analysis Kit for sizing. Molarity was calculated for each sample using average size as reported by Bioanalyzer or Fragment Analyzer and $\mathrm{pg} / \mu \mathrm{l}$ concentration as determined by PicoGreen. Samples were normalized to 2$10 \mathrm{nM}$ with Nuclease-free Water (Ambion). Libraries were multiplexed at 96 samples/lane and sequenced on an Illumina HiSeq 2500 instrument using Illumina High Output V4 chemistry.

\section{RNA-seq gene expression quantification}

1048 Raw read (fastq) files were aligned to the GRCh38 human genome sequence (Genome 4/13/2015) and updated by removing duplicate Entrez gene entries from the gtf reference file for STAR processing. For alignment, Illumina sequencing adapters were clipped from the reads using the fastqMCF program ${ }^{73}$. After clipping, the paired-end reads were mapped using Spliced Transcripts Alignment to a Reference (STAR) ${ }^{74}$ using default settings. STAR uses and builds it own suffix array index which considerably accelerates the alignment step while improving on sensitivity and specificity, due to its identification of alternative splice junctions. Reads that did 
not map to the genome were then aligned to synthetic constructs (i.e. ERCC) sequences and the E. coli genome (version ASM584v2). The final results files included quantification of the mapped reads (raw exon and intron counts for the transcriptome-mapped reads). Also, part of the final results files are the percentages of reads mapped to the RefSeq transcriptome, to ERCC spike-in controls, and to E. coli. Quantification was performed using summerizeOverlaps from the $R$ package GenomicAlignments ${ }^{75}$. Read alignments to the genome (exonic, intronic, and intergenic counts) were visualized as beeswarm plots using the R package beeswarm.

1063

1064

1065

1066

1067

1068

1069

1070

1071

1072

\section{Quality control of RNA-seq data}

1074 Nuclei were included for clustering analysis if they passed all of the following quality control 1075 (QC) thresholds:

$1076 \quad>30 \%$ cDNA longer than 400 base pairs

$1077 \quad$ - $\quad>500,000$ reads aligned to exonic or intronic sequence

$1078 \quad-\quad>40 \%$ of total reads aligned

$1079 \quad>50 \%$ unique reads

1080 - TA nucleotide ratio $>0.7$

1081

1082

1083

1084

1085

1086

1087

1088

1089

1090

1091

1092

1093

1094

1095

1096
After clustering (see below), clusters were identified as outliers if more than half of nuclei coexpressed markers of inhibitory (GAD1, GAD2) and excitatory (SLC17A7) neurons or were NeuN+ but did not express the pan-neuronal marker SNAP25. Median values of QC metrics listed above were calculated for each cluster and used to compute the median and inter-quartile range (IQR) of all cluster medians. Clusters were also identified as outliers if the cluster median QC metrics deviated by more than three times the IQRs from the median of all clusters.

Clusters were identified as donor-specific if they included fewer nuclei sampled from donors than expected by chance. For each cluster, the expected proportion of nuclei from each donor was calculated based on the laminar composition of the cluster and laminar sampling of the donor. For example, if $30 \%$ of layer 3 nuclei were sampled from a donor, then a layer 3-enriched cluster should contain approximately $30 \%$ of nuclei from this donor. In contrast, if only layer 5 were sampled from a donor, then the expected sampling from this donor for a layer 1-enriched cluster was zero. If the difference between the observed and expected sampling was greater than $50 \%$ of the number of nuclei in the cluster, then the cluster was flagged as donor-specific and excluded. 
To confirm exclusion, clusters automatically flagged as outliers or donor-specific were manually inspected for expression of broad cell class marker genes, mitochondrial genes related to quality, and known activity-dependent genes.

\section{Clustering RNA-seq data}

1102 Nuclei and cells were grouped into transcriptomic cell types using an iterative clustering procedure based on community detection in a nearest neighbor graph as described in ${ }^{33}$. Briefly, intronic and exonic read counts were summed, and log2-transformed expression (CPM + 1) was centered and scaled across nuclei. X-and Y-chromosome were excluded to avoid nuclei clustering based on sex. Many mitochondrial genes had expression that was correlated with RNA-seq data quality, so nuclear and mitochondrial genes downloaded from Human MitoCarta2.0 ${ }^{76}$ were excluded. Differentially expressed genes were selected while accounting for gene dropouts, and principal components analysis (PCA) was used to reduce dimensionality. Nearest-neighbor distances between nuclei were calculated using up to 20 principal components, Jaccard similarity coefficients were computed, and Louvain community detection was used to cluster this graph with 15 nearest neighbors. Marker genes were defined for all cluster pairs using two criteria: 1) significant differential expression (Benjamini-Hochberg false discovery rate $<0.05)$ using the $R$ package limma and 2$)$ either binary expression $(C P M>1$ in $>50 \%$ nuclei in one cluster and $<10 \%$ in the second cluster) or $>100$-fold difference in expression. Pairs of clusters were merged if either cluster lacked at least one marker gene.

1118 Clustering was then applied iteratively to each sub-cluster until the occurrence of one of four stop criteria: 1) fewer than six nuclei (due to a minimum cluster size of three); 2) no significantly variable genes; 3) no significantly variable PCs; 4) no significant clusters.

To assess the robustness of clusters, the iterative clustering procedure described above was repeated 100 times for random subsets of $80 \%$ of nuclei. A co-clustering matrix was generated that represented the proportion of clustering iterations that each pair of nuclei were assigned to the same cluster. We defined consensus clusters by iteratively splitting the co-clustering matrix as described in ${ }^{26}$. We used the co-clustering matrix as the similarity matrix and clustered using either Louvain (>= 4000 nuclei) or Ward's algorithm ( $<4000$ nuclei). We defined $N_{k, l}$ as the average probabilities of nuclei within cluster $k$ to co-cluster with nuclei within cluster $I$. We merged clusters $k$ and $/$ if $N_{k, l}>\max \left(N_{k, k}, N_{l, l}\right)-0.25$ or if the sum of -log10(adjusted P-value) of differentially expressed genes between clusters $k$ and $/$ was less than 150 . Finally, we refined cluster membership by reassigning each nucleus to the cluster to which it had maximal average co-clustering. We repeated this process until cluster membership converged.

Cluster names were defined using an automated strategy which combined molecular information (marker genes) and anatomical information (layer of dissection). Clusters were assigned a broad class of interneuron, excitatory neuron, microglia, astrocyte, oligodendrocyte precursor, oligodendrocyte, or endothelial cell based on maximal median cluster CPM of GAD1, SLC17A7, TYROBP, AQP4, PDGFRA, OPALIN, or NOSTRIN, respectively. Enriched layers were defined as the range of layers which contained at least $10 \%$ of the total cells from that cluster. Clusters in were then assigned a broad marker, defined by maximal median CPM of PAX6, LAMP5, VIP, SST, PVALB, LINC00507, RORB, THEMIS, FEZF2, TYROBP, FGFR3, PDGFRA, OPALIN, or NOSTRIN. Finally, clusters in all broad classes with more than one cluster (e.g., interneuron, excitatory neuron, and astrocyte) were assigned a gene showing the most specific expression in that cluster. These marker genes had the greatest difference in the proportion of expression (CPM > 1) with a cluster compared to all other clusters regardless of mean expression level. 


\section{Scoring cluster marker genes}

1146 Many genes were expressed in the majority of nuclei in a subset of clusters. A marker score (beta) was defined for all genes to measure how binary expression was among clusters, independent of the number of clusters labeled (Supplementary Table 2). First, the proportion

1150 $\left(x_{i}\right)$ of nuclei in each cluster that expressed a gene above background level $(C P M>1)$ was

1151 calculated. Then, scores were defined as the squared differences in proportions normalized by the sum of absolute differences plus a small constant $(\varepsilon)$ to avoid division by zero. Scores ranged from 0 to 1 , and a perfectly binary marker had a score equal to 1 .

$$
\beta=\frac{\sum_{i=1}^{n} \sum_{j=1}^{n}\left(x_{i}-x_{j}\right)^{2}}{\sum_{i=1}^{n} \sum_{j=1}^{n}\left|x_{i}-x_{j}\right|+\epsilon} .
$$

\section{Assigning core and intermediate cells}

1157 We defined core and intermediate cells as described ${ }^{26}$. Specifically, we used a nearest-centroid classifier, which assigns a cell to the cluster whose centroid has the highest Pearson's correlation with the cell. Here, the cluster centroid is defined as the median expression of the 1200 marker genes with the highest beta score. To define core vs. intermediate cells, we performed 5-fold cross-validation 100 times. In each round, the cells were randomly partitioned into 5 groups, and cells in each group of $20 \%$ of the cells were classified by a nearest centroid classifier trained using the other $80 \%$ of the cells. A cell classified to the same cluster as its original cluster assignment more than 90 times was defined as a core cell, the others were designated intermediate cells. We define 14,204 core cells and 1,399 intermediate cells, which in most cases classify to only 2 clusters $(1,345$ out of $1,399,96.1 \%)$. Most cells are defined as intermediate because they are confidently assigned to a different cluster from the one originally assigned $(1,220$ out of $1,399,87.2 \%)$ rather than because they are not confidently assigned to any cluster.

1171 Clusters were arranged by transcriptomic similarity based on hierarchical clustering. First, the average expression level of the top 1200 marker genes (highest beta scores, as above) was calculated for each cluster. A correlation-based distance matrix $\left(D_{x y}=\frac{1-\rho(x, y)}{2}\right)$ was calculated, and complete-linkage hierarchical clustering was performed using the "hclust" $R$ function with default parameters. The resulting dendrogram branches were reordered to show inhibitory clusters followed by excitatory clusters, with larger clusters first, while retaining the tree structure. Note that this measure of cluster similarity is complementary to the co-clustering separation described above. For example, two clusters with similar gene expression patterns but a few binary marker genes may be close on the tree but highly distinct based on coclustering.

\section{Mapping cell types to reported clusters}

118269 neuronal clusters in MTG were matched to 16 neuronal clusters reported by ${ }^{31}$ using nearestcentroid classifier of expression signatures. Specifically, single nucleus expression data was downloaded for 3042 single cells and 25,051 genes. 1359 marker genes (beta score $>0.4$ ) of 
1187 correlations were calculated between each nucleus in the Lake et al. dataset and all 69 MTG

1188

1189

1190

1191

1192

1193

1194

1195

1196

1197

1198

1199

1200

1201

1202

1203

1204

1205

1206

1207

1208

1209

1210

1211

1212

1213

1214

1215

1216

1217

1218

1219

1220

1221

1222

1223

1224

1225

1226

1227

1228

1229

1230

1231

clusters based on these 1359 genes. Nuclei were assigned to the cluster with the maximum correlation. A confusion matrix was generated to compare the cluster membership of nuclei reported by Lake et al. and assigned MTG cluster. The proportion of nuclei in each MTG cluster that were members of each of the 16 Lake et al. clusters were visualized as a dot plot with circle sizes proportional to frequency and colored by MTG cluster color.

\section{Colorimetric in situ hybridization}

In situ hybridization (ISH) data for human and mouse cortex was from the Allen Human Brain Atlas and Allen Mouse Brain Atlas. All ISH data is publicly accessible at: www.brain-map.org. Data was generated using a semi-automated technology platform as described ${ }^{77}$, with modifications for postmortem human tissues as previously described ${ }^{20}$. Digoxigenin-labeled riboprobes were generated for each human gene such that they would have $>50 \%$ overlap with the orthologous mouse gene in the Allen Mouse Brain Atlas ${ }^{77}$.

\section{GFAP immunohistochemistry}

Tissue slices $(350 \mu \mathrm{m})$ from neurosurgical specimens were fixed for $2-4$ days in $4 \%$ paraformaldehyde in PBS at $4^{\circ} \mathrm{C}$, washed in PBS, and cryoprotected in $30 \%$ sucrose. Cryoprotected slices were frozen and re-sectioned at $30 \mu \mathrm{m}$ using a sliding microtome (Leica SM2000R). Free floating sections were mounted onto gelatin coated slides and dried overnight at $37^{\circ} \mathrm{C}$. Slides were washed in $1 \mathrm{X}$ tris buffered saline (TBS), followed by incubation in $3 \%$ hydrogen peroxide in 1X TBS. Slides were then heated in sodium citrate $(\mathrm{pH} 6.0)$ for 20 minutes at $98^{\circ} \mathrm{C}$. After cooling, slides were rinsed in MilliQ water followed by $1 \mathrm{X}$ TBS. Primary antibody (mouse anti-GFAP, EMD Millipore, \#MAB360, clone GA5, 1:1500) was diluted in Renaissance Background Reducing Diluent (Biocare \#PD905L). Slides were processed using a Biocare intelliPATH FLX Automated Slide Stainer. After primary antibody incubation, slides were incubated in Mouse Secondary Reagent (Biocare \#IPSC5001G20), rinsed with 1X TBS, incubated in Universal HRP Tertiary Reagent (Biocare \#IPT5002G20), rinsed in 1X TBS, and incubated in IP FLXDAB (Biocare Buffer \#IPBF5009G20), and DAB chromogen (Biocare Chromogen \#IPC5008G3). Slides were then rinsed in 1X TBS, incubated in DAB sparkle (Biocare \#DSB830M), washed in MilliQ water, dehydrated through a series of graded alcohols, cleared with Formula 83, and coverslipped with DPX. Slides were imaged using an Aperio ScanScope XT slide scanner (Leica).

\section{Multiplex fluorescence in situ hybridization (FISH)}

Genes were selected for multiplex FISH experiments that discriminated cell types and broader classes by visual inspection of differentially expressed genes that had relatively binary expression in the targeted types.

\section{Single molecule FISH (smFISH)}

Fresh-frozen human brain tissue from the MTG was sectioned at 10um onto Poly-L-lysine coated coverslips as described previously ${ }^{78}$, let dry for $10 \mathrm{~min}$ at room temperature, then fixed for $15 \mathrm{~min}$ at $4 \mathrm{C}$ in $4 \%$ PFA. Sections were washed $3 \times 10 \mathrm{~min}$ in PBS, then permeabilized and dehydrated with $100 \%$ isopropanol at room temperature for $3 \mathrm{~min}$ and allowed to dry. Sections were stored at $-80 \mathrm{C}$ until use. Frozen sections were rehydrated in 2XSSC (Sigma Aldrich 20XSSC, 15557036) for $5 \mathrm{~min}$, then treated $2 \times 5$ min with 4\%SDS (Sigma Aldrich, 724255) and $200 \mathrm{mM}$ boric acid (Sigma Aldrich, cat\# B6768) pH 8.5 at room temperature. Sections were washed 3 times in 2X SSC, then once in TE pH 8 (Sigma Aldrich, 93283). Sections were heatshocked at $70 \mathrm{C}$ for $10 \mathrm{~min}$ in $\mathrm{TE} \mathrm{pH} 8$, followed by 2XSSC wash at room temperature. 
1232 Sections were then incubated in hybridization buffer (10\% Formamide (v/v, Sigma Aldrich

1233 4650), 10\% Dextran Sulfate (w/v, Sigma Aldrich D8906), 200ug/mL BSA (Ambion AM2616), 2 mM Ribonucleoside vanadyl complex (New England Biolabs, S1402S), 1mg/ml tRNA (Sigma

1234

1235

1236

1237

1238

1239

1240

1241

1242

1243 10109541001 ) in 2XSSC) for $5 \mathrm{~min}$ at $38.5 \mathrm{C}$. Probes were diluted in hybridization buffer at a concentration of $250 \mathrm{nM}$ and hybridized at $38.5 \mathrm{C}$ for $2 \mathrm{~h}$. Following hybridization, sections were washed $2 \times 15 \mathrm{~min}$ at $38.5 \mathrm{C}$ in wash buffer (2XSSC, 20\% Formamide), and 1 X 15 min in wash buffer with $5 \mathrm{ug} / \mathrm{ml}$ DAPI (Sigma Aldrich, 32670). Sections are then imaged in Imaging buffer (20 mM Tris-HCl pH 8, $50 \mathrm{mM} \mathrm{NaCl}, 0.8 \%$ Glucose (Sigma Aldrich, G8270), $3 \mathrm{U} / \mathrm{ml}$ Glucose Oxidase (Sigma Aldrich, G2133), $90 \mathrm{U} / \mathrm{ml}$ Catalase (Sigma Aldrich, C3515). Following imaging, sections were incubated $3 \times 10$ min in stripping buffer (65\% Formamide, 2 X SSC) at $30 \mathrm{C}$ to remove hybridization probes from the first round. Sections were then washed in $2 X$ SSC for $3 X$ $5 \mathrm{~min}$ at room temperature prior to repeating the hybridization procedure.

\section{RNAscope multiplex FISH}

Human tissue specimens used for RNAscope multiplex FISH came from either neurosurgical resections or postmortem brain specimens. Mouse tissue for RNAscope experiments was from adult (P56 +/- 3 days) wildtype C57BI/6J mice. All animal procedures were approved by the Institutional Animal Care and Use Committee at the Allen Institute for Brain Science (Protocol No. 1511). Mice were provided food and water ad libitum, maintained on a regular $12-\mathrm{h}$ day/night cycle, and housed in cages with various enrichment materials added, including nesting materials, gnawing materials, and plastic shelters. Mice were anesthetized with $5 \%$ isoflurane and intracardially perfused with either 25 or $50 \mathrm{ml}$ of ice cold, oxygenated artificial

1253

1254 cerebral spinal fluid $(0.5 \mathrm{mM} \mathrm{CaCl} 2,25 \mathrm{mM}$ D-Glucose, $98 \mathrm{mM} \mathrm{HCl}, 20 \mathrm{mM}$ HEPES, $10 \mathrm{mM}$ MgSO4, 1.25mM NaH2PO4, 3mM Myo-inositol, 12mM N-acetylcysteine, 96mM N-methyl-Dglucamine, $2.5 \mathrm{mM} \mathrm{KCl}, 25 \mathrm{mM} \mathrm{NaHCO}, 5 \mathrm{mM}$ sodium L-Ascorbate, $3 \mathrm{mM}$ sodium pyruvate, $0.01 \mathrm{mM}$ Taurine, and $2 \mathrm{mM}$ Thiourea). The brain was then rapidly dissected, embedded in optimal cutting temperature (O.C.T.) medium, and frozen in a slurry of dry ice and ethanol. Tissues were stored at $-80 \mathrm{C}$ until for later cryosectioning.

\section{RNAscope multiplex FISH with GFAP immunohistochemistry}

1268 Tissue sections were processed for RNAscope multiplex FISH detection of ID3 (ACD Bio,

Fresh-frozen mouse or human tissues were sectioned at 14-16um onto Superfrost Plus glass slides (Fisher Scientific). Sections were dried for 20 minutes at $-20 \mathrm{C}$ and then vacuum sealed and stored at $-80 \mathrm{C}$ until use. The RNAscope multiplex fluorescent $\mathrm{v} 1 \mathrm{kit}$ was used according to the manufacturer's instructions for fresh-frozen tissue sections (ACD Bio), with the following minor modifications: (1) fixation was performed for 60 minutes in $4 \%$ paraformaldehyde in $1 X$ PBS at $4{ }^{\circ} \mathrm{C}$, and (2) the protease treatment step was shortened to $15 \mathrm{~min}$. Sections were imaged using either a 40X or 60X oil immersion lens on a Nikon TiE fluorescent microscope equipped with NIS-Elements Advanced Research imaging software (version 4.20).

1273

1274

1275

1276

1277 \#492181-C3, NM_002167.4 ) and AQP4 (ACD Bio, \#482441, NM_001650.5 ) exactly as described above. At the end of the RNAscope protocol, sections were fixed in $4 \%$ paraformaldehyde for 15 minutes at room temperature and then washed twice in 1X PBS for 5 minutes. Sections were incubated in blocking solution $(10 \%$ normal donkey serum, $0.1 \%$ triton- $X$ 100 in 1X PBS) for 30 minutes at room temperature and then incubated in primary antibody diluted 1:100 in blocking solution (mouse anti-GFAP, Sigma-Aldrich, \#G3893, clone G-A-5) for 18 hours at 4C. Sections were then washed 3 times for 5 minutes each in 1X PBS, incubated with secondary antibody (goat anti-mouse $\lg \mathrm{G}(\mathrm{H}+\mathrm{L})$ Alexa Fluor 568 conjugate, ThermoFisher Scientific, \#A-11004) for 30 minutes at room temperature, rinsed in 1X PBS 3 times for 5 1278 minutes each, counterstained with DAPI $(1 \mathrm{ug} / \mathrm{ml})$, and mounted with ProLong Gold mounting 
medium (ThermoFisher Scientific). Sections were imaged using either a 40X or 60X oil immersion lens on a Nikon TiE fluorescent microscope equipped with NIS-Elements Advanced Research imaging software (version 4.20).

\section{In situ validation of excitatory types}

To validate excitatory neuron types, clusters were labeled with cell type specific combinatorial gene panels. For each gene panel, positive cells were manually called by visual assessment of RNA spots for each gene. The total number of positive cells was quantified for each section. Cells were counted on at least three sections derived from at least two donors for each probe combination. DAPI staining was used to determine the boundaries of cortical layers within each tissue section and the laminar position of each positive cell was recorded. The percentage of labeled cells per layer, expressed as a fraction of the total number of labeled cells summed across all layers, was calculated for each type. Probes used in these experiments were as follows (all from ACD Bio): SLC17A7 (\#415611, NM_020309.3 ), RORB (\#446061, \#446061C2, NM_006914.3), CNR1 (\#591521-C2, NM_001160226.1), PRSS12 (\#493931C3, NM_003619.3), ALCAM (\#415731-C2, NM_001243283.1), MET (\#431021, NM_001127500.1), MME (\#410891-C2, NM_007289.2 ), NTNG1 (\#446101C3, NM_001113226.1), HS3ST4 (\#506181, NM_006040.2), CUX2 (\#425581C3, NM_015267.3), PCP4 (\#446111, NM_006198.2), GRIN3A (\#534841-C3, NM_133445.2), GRIK3 (\#493981, NM_000831.3), CRHR2 (\#469621, NM_001883.4), TPBG (\#405481, NM_006670.4), POSTN (\#409181-C3, NM_006475.2), SMYD1 (\#493951-

\section{In situ validation of putative chandelier cells}

1301 Tissue sections were labeled with the gene panel GAD1, PVALB, and NOG, or COL15A1, specific markers of the Inh L2-5 PVALB SCUBE3 putative chandelier cell cluster. Probes were as follows (all from ACD Bio): GAD1 (\#404031-C3, NM_000817.2), PVALB (\#422181C2, NM_002854.2), NOG (\#416521, NM_005450.4), CŌL15A1 (\#484001, NM_001855.4). Counts were conducted on sections from 3 human tissue donors. For each donor, the total number of GAD1+, PVALB+ and NOG+ cells was summed across multiple sections. The laminar position of each cell, based on boundaries defined by assessing DAPI staining patterns in each tissue section, was recorded. The proportion of chandelier cells in each layer was calculated as a fraction of the total number of $G A D 1+/ P V A L B+/ N O G+$ cells summed across all layers for each specimen.

\section{Cell counts of broad interneuron classes}

1312 Tissue sections were labeled with the RNAscope Multiplex Fluorescent kit (ACD Bio) as

1313

1314

1315

1316

1317

1318

1319

1320

1321

1322

1323 described above. For human tissue sections, the following probes (all from ACD Bio) were used: GAD1 (\#404031, NM_000817.2); ADARB2 (\#511651-C3, NM_018702.3); LHX6 (\#460051-C2, NM_014368.4). For mouse tissue sections, the following probes were used: Gad1 (\#400951, NM_008077.4); Adarb2 (\#519971-C3, NM_052977.5); Lhx6 (\#422791-C2, NM_001083127.1). The expression of each gene was assessed by manual examination of corresponding RNA spots. Cell counts were conducted on sections from 3 human tissue donors: 2 neurosurgical and 1 postmortem. For mouse, 3 independent specimens were used. For both human and mouse, $>500$ total GAD1+ cells per specimen were counted (Human, $\mathrm{n}=2706,1553$, and 3476 GAD1+ cells per donor, respectively; Mouse, $\mathrm{n}=1897,2587$, and 708 GAD1+ cells per specimen, resepectively). Expression of $A D A R B 2 / A d a r b 2$ and $L H X 6 / L h \times 6$ was manually assessed in each GAD1+ cell and cells were scored as being positive or negative for each

1324 gene. At the same time, the laminar position of each GAD1+ cell was recorded. Cell density, 
1325 highlighted by DAPI staining, was used to determine laminar boundaries. The percentage of each cell class expressed as a fraction of total GAD1+ cells and the percentage of each cell class per layer, expressed as a fraction of the total number of GAD1+ cells per layer, were calculated for each specimen. Statistical comparisons between human and mouse were done using unpaired two-tailed t-tests with Holm-Sidak correction for multiple comparisons.

\section{Imaging and quantification of smFISH expression}

smFISH images were collected using an inverted microscope in an epifluorescence configuration (Zeiss Axio Observer.Z1) with a 63x oil immersion objective with numerical aperture 1.4. The sample was positioned in $x, y$ and $z$ with a motorized $x, y$ stage with linear encoders and $z$ piezo top-plate (Applied Scientific Instruments MS 2000-500) and z stacks with $300 \mathrm{~nm}$ plane spacing were collected in each color at each stage position through the entire $z$ depth of the sample. Fluorescence emission was filtered using a high-speed filterwheel (Zeiss) directly below the dichroic turret and imaged onto a sCMOS camera (Hamamatsu ORCA Flash4.0) with a final pixel size of $100 \mathrm{~nm}$. Images were collected after each round of hybridization using the same configuration of $x$, $y$ tile locations, aligned manually before each acquisition based on DAPI fluorescence. smFISH signal was observed as diffraction-limited spots which were localized in 3D image stacks by finding local maxima after spatial bandpass filtering. These maxima were filtered for total intensity and radius to eliminate dim background and large, bright lipofuscin granules. Outlines of cells and cortical layers were manually annotated on images of GAD, SLC17A7 and DAPI as 2D polygons using FIJI. The number of mRNA molecules in each cell for each gene was then calculated and converted to densities (spots per 100um²).

Background expression of the excitatory neuron marker SLC17A7 was defined as the 95th quantile of SLC17A7 spot density among cells in cortical layer 1, since no excitatory cells should be present in layer 1. Excitatory neurons were defined as any cell with SLC17A7 spot density greater than this threshold. To map excitatory cells to MTG reference clusters, spot counts were log-transformed and scaled so that the 90th quantile of expression for each gene in smFISH matched the maximum median cluster expression of that gene among the reference clusters. Reference clusters that could not be discriminated based on the smFISH panel of nine genes were merged and all comparisons between smFISH and RNA-seq cluster classes were performed using these cluster groups. Scaled spot densities for each cell were then compared to median expression levels of each reference cluster using Pearson correlation, and each cell was assigned to the cluster with the highest correlation. For cells that mapped to the Exc L23 LINC00507 FREM3 cluster, LAMP5 and COL5A2 expression was plotted as a dot plot where the size and color of dots corresponded to probe spot density and the location corresponded to 1361 the in situ location.

\section{MetaNeighbor analysis}

1363 To compare the ability of different gene sets to distinguish cell types in mouse versus human 1364 cortex, we performed a modified supervised MetaNeighbor analysis ${ }^{79}$ independently for both species. First, we divided our data sets into two artificial experiments, selecting random groups of equal size up to a maximum of 10 cells per cluster for each experiment. We next ran MetaNeighbor separately for clusters from each broad class (GABAergic, glutamatergic, and non-neuronal) using the R function "run_MetaNeighbor" where "experiment_labels" are 1 or 2 corresponding to the two artificial experiments, "celltype_labels" are 2 for cells in the targeted cluster and 1 for cells in all other clusters of the same broad class, and "genesets" were all of the HGNC gene sets included in Table S3 of ${ }^{52}$. Mean AUROC scores for each gene set were 
1372 then calculated by averaging the reported AUROC scores for a gene set across all clusters within a given broad class. This processes was repeated for 10 divisions of the human and mouse data into random experimental groups. Means and standard deviations of these mean AUROC scores for human and mouse GABAergic cell types are compared in Fig $\mathbf{5 .}$

\section{Estimation of cell type homology}

1377 We aligned single nucleus and single cell RNA-seq data from human MTG and mouse primary visual cortex by applying canonical correlation analysis (CCA) as implemented in the Seurat $R$ package ${ }^{56}$. We used log2-transformed CPM of intronic plus exonic reads for both datasets. Including exonic reads increased experimental differences due to measuring whole cell versus nuclear transcripts, but this was out-weighed by improved gene detection. We separated each of the datasets into three broad cell classes: GABAergic, glutamatergic, and non-neuronal, based on their assigned clusters, and selected up to 200 cells from each cluster. We included mouse non-neuronal cells from cell types that we had captured in our human survey, including astrocytes, oligodendrocyte precursors, oligodendrocytes, endothelial cells, and microglia. For each of these datasets, we selected the union of the top 2,000 genes with the highest dispersion for human and mouse and calculated 40 canonical correlates with diagonal CCA. Following this step, we removed 88 nuclei or cells for which the variance explained by CCA was less than half of the variance explained by PCA, and aligned the canonical basis vectors to allow integrated analysis. In particular, all human endothelial nuclei and over half of human microglial nuclei were removed along with mouse Cajal-Retzius cells.

We defined homologous cell types by clustering canonical correlates and identifying human and mouse samples that co-clustered. Initially, the first 10 canonical correlates were selected, and a weighted graph was constructed based on the Jaccard similarity of the 10 nearest neighbors of each sample. Louvain community detection was run to identify clusters that optimized the global modularity of the partitioned graph. For each pair of human and mouse clusters, the overlap was defined as the sum of the minimum proportion of samples in each cluster that overlapped within each CCA cluster. This approach identified pairs of human and mouse clusters that consistently co-clustered within one or more CCA clusters. Cluster overlaps varied from 0 to 1 and were visualized as a heatmap with human clusters as rows and mouse clusters as columns. Cell type homologies were identified as one-to-one, one-to-many, or many-to-many based on the pattern of overlap between clusters. A quality score was calculated for the homology mapping that rewarded overlaps greater than 0.6 ( 0.2 for non-neuronal clusters) and penalized for clusters lacking any overlaps. For each human cluster, the inverse of the sum of the number of overlapping mouse clusters was calculated, and this value was set to -1 if no overlapping clusters were found. The quality score was defined as the sum of the scores for the individual clusters and could range from -38 (no overlap) to 38 (all one-to-one matches). Including more canonical correlates or fewer nearest neighbors increased the number of cell types that could be discriminated within each species (increasing the quality score) but also resulted in more species-specific clusters (decreasing the quality score). A grid based search was used to select the number of canonical correlates and nearest neighbors that maximized the quality score.

1414 1).

\section{Quantification of expression divergence}

1416 For each pair of 38 homologous human and mouse cell types, the average expression of 14,414 
1419 (mouse) datasets. Average expression values were log2-transformed and scatter plots and Pearson's correlations were calculated to compare human and mouse. Genes were ranked based on their cell type-specificity in human and mouse using a tau score defined in ${ }^{80}$, and the union of the top 50 markers in human and mouse were highlighted in the scatter plots. The fold difference in expression between human and mouse was calculated for all genes and homologous cell types and thresholded to identify large (>10-fold), moderate (2- to 10-fold), and small (<2-fold) differences. A heatmap was generated showing expression differences across cell types, and hierarchical clustering using Ward's method was applied to group genes with similar patterns of expression change. For each of 6 major classes of cell types (LAMP5/PAX6, VIP, SST, PVALB, excitatory, non-neuronal), the number of genes was quantified that had $>10$-fold change in at least one cell type in that class and $<10$-fold change in all cell types in the other 5 classes. The expression pattern change of 14,414 genes was quantified as the beta score (see marker score methods above) of log2-expression differences across 38 homologous cell types (Supplementary Table 2). Genes with high scores have a large fold-change in expression in one or more (but not all) cell types. For each gene, the number of clusters with median expression (CPM) > 1 was compared to the median pattern change of those genes. A loess curve and standard error were fit using the R package ggplot. Finally, the median pattern change was calculated for the functional gene families used in the MetaNeighbor analysis described above.

\section{Data and Code Availability}

1439 Data and code used to produce figures will be available from https://github.com/AllenInstitute/MTG_celltypes. RNA-seq data from this study is publicly available and can be downloaded at http://celltypes.brain-map.org/, and data can be visualized and analyzed using two complementary viewers at http://celltypes.brainmap.org/rnaseq/human and https://viewer.cytosplore.org/.

\section{References}

1445 1.Glasser, M. F. et al. A multi-modal parcellation of human cerebral cortex. Nature 536, 1711446178 (2016).

1447 2.Nieuwenhuys, R. The myeloarchitectonic studies on the human cerebral cortex of the Vogt1448 Vogt school, and their significance for the interpretation of functional neuroimaging data. Brain 1449 Struct Funct 218, 303-52 (2013).

1450 3.Essen, D. C. V., Glasser, M. F., Dierker, D. L., Harwell, J. \& Coalson, T. Parcellations and 1451 Hemispheric Asymmetries of Human Cerebral Cortex Analyzed on Surface-Based Atlases. 1452 Cerebral Cortex 22, 2241-2262 (2011).

1453 4.Azevedo, F. A. C. et al. Equal numbers of neuronal and nonneuronal cells make the human 1454 brain an isometrically scaled-up primate brain. The Journal of Comparative Neurology 513, 1455 532-541 (2009).

1456 5.Herculano-Houzel, S., Mota, B. \& Lent, R. Cellular scaling rules for rodent brains. Proc Natl 1457 Acad Sci U S A 103, 12138-43 (2006).

1458 6.Azevedo, F. A. et al. Equal numbers of neuronal and nonneuronal cells make the human brain 1459 an isometrically scaled-up primate brain. J Comp Neurol 513, 532-41 (2009). 
1460 7.Geschwind, D. H. \& Rakic, P. Cortical evolution: judge the brain by its cover. Neuron 80, 633146147 (2013).

1462 8.DeFelipe, J. The evolution of the brain, the human nature of cortical circuits, and intellectual

1463 creativity. Front Neuroanat 5, 29 (2011).

1464 9.Cajal, S. Ramón y. La Textura del Sistema Nerviosa del Hombre y los Vertebrados. (1904).

1465 10.Nó, R. Lorente de. La corteza cerebral del ratón. Trab. Lab. Invest. Bio. (Madrid) 20, (1922).

1466 11.Poorthuis, R. B. et al. Rapid Neuromodulation of Layer 1 Interneurons in Human Neocortex.

1467 Cell Rep 23, 951-958 (2018).

1468 12.Eyal, G. et al. Unique membrane properties and enhanced signal processing in human

1469 neocortical neurons. Elife 5, (2016).

1470 13.Szegedi, V. et al. Plasticity in Single Axon Glutamatergic Connection to GABAergic

1471 Interneurons Regulates Complex Events in the Human Neocortex. PLoS Biol 14, e2000237

1472 (2016).

1473 14.DeFelipe, J. Types of neurons, synaptic connections and chemical characteristics of cells 1474 immunoreactive for calbindin-D28K, parvalbumin and calretinin in the neocortex. J Chem

1475 Neuroanat 14, 1-19 (1997).

1476 15.Benavides-Piccione, R., Ballesteros-Yáñez, I., DeFelipe, J. \& Yuste, R. Cortical area and 1477 species differences in dendritic spine morphology. J Neurocytol 31, 337-46 (2002).

1478 16.Gabbott, P. L. Subpial Fan Cell - A Class of Calretinin Neuron in Layer 1 of Adult Monkey 1479 Prefrontal Cortex. Front Neuroanat 10, 28 (2016).

1480 17.Oberheim, N. A. et al. Uniquely hominid features of adult human astrocytes. J Neurosci 29, $14813276-87$ (2009).

1482 18.Hill, R. S. \& Walsh, C. A. Molecular insights into human brain evolution. Nature 437, 64-7

1483 (2005).

1484 19.Boldog, E. et al. Transcriptomic and morphophysiological evidence for a specialized human 1485 cortical GABAergic cell type. bioRxiv (2017). doi:10.1101/216085

1486 20.Zeng, H. et al. Large-scale cellular-resolution gene profiling in human neocortex reveals 1487 species-specific molecular signatures. Cell 149, 483-96 (2012).

1488 21.Bakken, T. E. et al. A comprehensive transcriptional map of primate brain development.

1489 Nature 535, 367-75 (2016).

1490 22. Hawrylycz, M. et al. Canonical genetic signatures of the adult human brain. Nat Neurosci 18, 1491 1832-44 (2015).

1492 23.Miller, J. A. et al. Transcriptional landscape of the prenatal human brain. Nature 508, 1991493206 (2014).

1494 24.Ecker, J. R. et al. The BRAIN Initiative Cell Census Consortium: Lessons Learned toward 1495 Generating a Comprehensive Brain Cell Atlas. Neuron 96, 542-557 (2017).

1496 25.Regev, A. et al. The Human Cell Atlas. Elife 6, (2017). 
1497 26. Tasic, B. et al. Shared and distinct transcriptomic cell types across neocortical areas. bioRxiv 1498 (2017). doi:10.1101/229542

1499 27.Tasic, B. et al. Adult mouse cortical cell taxonomy revealed by single cell transcriptomics.

1500 Nat Neurosci 19, 335-46 (2016).

1501 28.Zeisel, A. et al. Brain structure. Cell types in the mouse cortex and hippocampus revealed by 1502 single-cell RNA-seq. Science 347, 1138-42 (2015).

1503 29.Darmanis, S. et al. A survey of human brain transcriptome diversity at the single cell level. 1504 Proc Natl Acad Sci U S A 112, 7285-90 (2015).

1505 30.Krishnaswami, S. R. et al. Using single nuclei for RNA-seq to capture the transcriptome of 1506 postmortem neurons. Nat Protoc 11, 499-524 (2016).

1507 31.Lake, B. B. et al. Neuronal subtypes and diversity revealed by single-nucleus RNA 1508 sequencing of the human brain. Science 352, 1586-90 (2016).

1509 32. Habib, N. et al. Massively parallel single-nucleus RNA-seq with DroNc-seq. Nat Methods 14, 1510 955-958 (2017).

1511 33.Bakken, T. E. et al. Equivalent high-resolution identification of neuronal cell types with single1512 nucleus and single-cell RNA-sequencing. bioRxiv (2017). doi:10.1101/239749

1513 34.Lake, B. B. et al. A comparative strategy for single-nucleus and single-cell transcriptomes 1514 confirms accuracy in predicted cell-type expression from nuclear RNA. Sci Rep 7, 6031 (2017).

1515 35.Lake, B. B. et al. Integrative single-cell analysis of transcriptional and epigenetic states in the 1516 human adult brain. Nat Biotechnol 36, 70-80 (2018).

1517 36.DeFelipe, J., Alonso-Nanclares, L. \& Arellano, J. I. Microstructure of the neocortex:

1518 comparative aspects. J Neurocytol 31, 299-316 (2002).

1519 37.Bakken, T. et al. Cell type discovery and representation in the era of high-content single cell 1520 phenotyping. BMC Bioinformatics 18, 559 (2017).

1521 38.Bakken, T. E. et al. Spatiotemporal dynamics of the postnatal developing primate brain

1522 transcriptome. Human Molecular Genetics 24, 4327-4339 (2015).

1523 39.Werner, M. S. et al. Chromatin-enriched IncRNAs can act as cell-type specific activators of 1524 proximal gene transcription. Nat Struct Mol Biol 24, 596-603 (2017).

1525 40.Von Economo, C. Cellular structure of the human cerebral cortex. (Karger Medical and 1526 Scientific Publishers, 2009).

1527 41.Kalmbach, B. et al. h-channels contribute to divergent electrophysiological properties of 1528 supragranular pyramidal neurons in human versus mouse cerebral cortex. bioRxiv (2018). 1529 doi:10.1101/312298

1530 42.Cytosplore: Interactive Immune Cell Phenotyping for Large Single-Cell Datasets. Computer 1531 Graphics Forum 35, (2016).

1532 43. Hollt, T. et al. CyteGuide: Visual Guidance for Hierarchical Single-Cell Analysis. IEEE Trans 1533 Vis Comput Graph 24, 739-748 (2018). 
1534 44.Lee, S., Hjerling-Leffler, J., Zagha, E., Fishell, G. \& Rudy, B. The largest group of superficial neocortical GABAergic interneurons expresses ionotropic serotonin receptors. $J$ Neurosci 30, 16796-808 (2010).

1537 45.Hansen, D. V. et al. Non-epithelial stem cells and cortical interneuron production in the 1538 human ganglionic eminences. Nat Neurosci 16, 1576-87 (2013).

1539 46.Ma, T. et al. Subcortical origins of human and monkey neocortical interneurons. Nat 1540 Neurosci 16, 1588-97 (2013).

1541 47.Tasic, B. et al. Shared and distinct transcriptomic cell types across neocortical areas. bioRxiv 1542 (2017). doi:10.1101/229542

1543 48.Shah, B. P. et al. MC4R-expressing glutamatergic neurons in the paraventricular

1544

1545 hypothalamus regulate feeding and are synaptically connected to the parabrachial nucleus. Proc Natl Acad Sci U S A 111, 13193-8 (2014).

1546 49.Horstmann, A. et al. Common genetic variation near MC4R has a sex-specific impact on 1547 human brain structure and eating behavior. PLoS One 8, e74362 (2013).

1548 50.Raghanti, M. A. et al. Neuropeptide Y-immunoreactive neurons in the cerebral cortex of humans and other haplorrhine primates. Am J Primatol 75, 415-24 (2013).

1551 51.Xu, X., Roby, K. D. \& Callaway, E. M. Immunochemical characterization of inhibitory mouse

1552 cortical neurons: three chemically distinct classes of inhibitory cells. J Comp Neurol 518, 389_ 404 (2010).

52.Paul, A. et al. Transcriptional Architecture of Synaptic Communication Delineates GABAergic Neuron Identity. Cell 171, 522-539.e20 (2017).

53. Marques, S. et al. Oligodendrocyte heterogeneity in the mouse juvenile and adult central nervous system. Science 352, 1326-1329 (2016). (2016).

55.Sosunov, A. A. et al. Phenotypic heterogeneity and plasticity of isocortical and hippocampal astrocytes in the human brain. J Neurosci 34, 2285-98 (2014). 411-420 (2018).

1567 58.He, M. et al. Strategies and Tools for Combinatorial Targeting of GABAergic Neurons in Mouse Cerebral Cortex. Neuron 92, 555 (2016).

1569 59.Sorensen, S. A. et al. Correlated Gene Expression and Target Specificity Demonstrate 1570 Excitatory Projection Neuron Diversity. Cerebral Cortex 25, 433-449 (2013).

1571 60.Belichenko, P. V., Vogt, W. D. M., Myklóssy, J. \& Celio, M. R. Calretinin-positive Cajal1572 Retzius cells persist in the adult human neocortex. Neuroreport 6, 1869-74 (1995). 
1573 61.Glezer, I. I., Hof, P. R. \& Morgane, P. J. Calretinin-immunoreactive neurons in the primary

1574 visual cortex of dolphin and human brains. Brain Res 595, 181-8 (1992).

1575 62.Miyoshi, G. et al. Genetic fate mapping reveals that the caudal ganglionic eminence

1576 produces a large and diverse population of superficial cortical interneurons. J Neurosci 30,

1577 1582-94 (2010).

1578 63.Lein, E., Borm, L. E. \& Linnarsson, S. The promise of spatial transcriptomics for

1579 neuroscience in the era of molecular cell typing. Science 358, 64-69 (2017).

1580 64.Colantuoni, C. et al. Temporal dynamics and genetic control of transcription in the human

1581 prefrontal cortex. Nature 478, 519-23 (2011).

1582 65.Kang, H. J. et al. Spatio-temporal transcriptome of the human brain. Nature 478, 483-489

1583 (2011).

1584 66.Bahney, J. \& Bartheld, C. S. von. The Cellular Composition and Glia-Neuron Ratio in the

1585 Spinal Cord of a Human and a Nonhuman Primate: Comparison with Other Species and Brain

1586 Regions. The Anatomical Record 301, 697-710 (2017).

1587 67.Bjugn, R. The use of the optical disector to estimate the number of neurons, glial and endothelial cells in the spinal cord of the mouse-with a comparative note on the rat spinal cord. Brain Res 627, 25-33 (1993).

1590 68.Lassek, A. M. \& Rassmussen, G. L. The human pyramidal tract: A fiber and numerical 1591 analysis. Archives of Neurology \& Psychiatry 42, 872-876 (1939).

1592 69.Finlay, B. \& Darlington, R. Linked regularities in the development and evolution of 1593 mammalian brains. Science 268, 1578-1584 (1995).

1594 70.Markou, A., Chiamulera, C., Geyer, M. A., Tricklebank, M. \& Steckler, T. Removing obstacles in neuroscience drug discovery: the future path for animal models. Neuropsychopharmacology

1596 34, 74-89 (2009).

1597 71.Nestler, E. J. \& Hyman, S. E. Animal models of neuropsychiatric disorders. Nature 1598 Neuroscience 13, 1161-1169 (2010).

1599 72.Sorensen, S. A. et al. Correlated Gene Expression and Target Specificity Demonstrate 1600 Excitatory Projection Neuron Diversity. Cerebral Cortex 25, 433-449 (2013).

1601 73.Aronesty, E. Comparison of Sequencing Utility Programs. The Open Bioinformatics Journal 1602 7, 1-8 (2013).

1603 74.Dobin, A. et al. STAR: ultrafast universal RNA-seq aligner. Bioinformatics 29, 15-21 (2012).

1604 75.Lawrence, M. et al. Software for Computing and Annotating Genomic Ranges. PLoS

1605 Computational Biology 9, e1003118 (2013).

1606 76.Calvo, S. E., Clauser, K. R. \& Mootha, V. K. MitoCarta2.0: an updated inventory of

1607 mammalian mitochondrial proteins. Nucleic Acids Res 44, D1251-7 (2016).

1608 77.Lein, E. S. et al. Genome-wide atlas of gene expression in the adult mouse brain. Nature 1609 445, 168-76 (2007).

1610 78.Lyubimova, A. et al. Single-molecule mRNA detection and counting in mammalian tissue.

1611 Nat Protoc 8, 1743-58 (2013). 
1612 79.Crow, M., Paul, A., Ballouz, S., Huang, Z. J. \& Gillis, J. Characterizing the replicability of cell 1613 types defined by single cell RNA-sequencing data using MetaNeighbor. Nat Commun 9, 884 1614 (2018).

1615 80.Yanai, I. et al. Genome-wide midrange transcription profiles reveal expression level 1616 relationships in human tissue specification. Bioinformatics 21, 650-9 (2005). 


\begin{tabular}{|c|c|c|c|c|c|c|c|c|c|c|}
\hline $\begin{array}{c}\text { Specimen } \\
\text { ID }\end{array}$ & $\begin{array}{c}\text { Tissue } \\
\text { Type }\end{array}$ & Age & Sex & Race & $\begin{array}{c}\text { Cause } \\
\text { of } \\
\text { Death }\end{array}$ & $\begin{array}{l}\text { PMI } \\
\text { (hr) }\end{array}$ & $\begin{array}{l}\text { Tissue } \\
\text { RIN }\end{array}$ & $\begin{array}{l}\text { Hemisphere } \\
\text { Sampled }\end{array}$ & $\begin{array}{l}\text { Reason for } \\
\text { Surgery }\end{array}$ & $\begin{array}{l}\text { Number } \\
\text { of nuclei } \\
\text { sampled }\end{array}$ \\
\hline H200.1023 & $P$ & 43 & $\mathrm{~F}$ & $\begin{array}{l}\text { Iranian } \\
\text { descent }\end{array}$ & $\begin{array}{l}\text { Mitral } \\
\text { valve } \\
\text { prolapse }\end{array}$ & 18.5 & $\begin{array}{l}7.4 \pm \\
0.7\end{array}$ & $\mathrm{~L}$ & $\mathrm{~N} / \mathrm{A}$ & 6170 \\
\hline H200.1025 & $P$ & 50 & $M$ & Caucasian & $\mathrm{CV}$ & 24.5 & $\begin{array}{l}7.6 \pm \\
1.0\end{array}$ & $\mathrm{~L}$ & $\mathrm{~N} / \mathrm{A}$ & 1334 \\
\hline $\mathrm{H} 200.1030$ & $\mathrm{P}$ & 54 & M & Caucasian & CV & 25 & $\begin{array}{l}7.7 \pm \\
0.8\end{array}$ & $\mathrm{~L}$ & $\mathrm{~N} / \mathrm{A}$ & 7331 \\
\hline H16.24.010 & $P$ & 66 & $\mathrm{M}$ & Caucasian & $\mathrm{CV}$ & 21 & $\begin{array}{l}7.2 \pm \\
1.4\end{array}$ & $\mathrm{~L}$ & $\mathrm{~N} / \mathrm{A}$ & 371 \\
\hline H16.06.002 & $\mathrm{N}$ & 35 & $\mathrm{~F}$ & Caucasian & $\mathrm{N} / \mathrm{A}$ & $\mathrm{N} / \mathrm{A}$ & $\begin{array}{l}7.1 \pm \\
0.9\end{array}$ & $\mathrm{R}$ & Epilepsy & 97 \\
\hline H16.06.008 & $\mathrm{N}$ & 24 & $F$ & Hispanic & $\mathrm{N} / \mathrm{A}$ & $\mathrm{N} / \mathrm{A}$ & $\begin{array}{l}8.1 \pm \\
0.8\end{array}$ & $\mathrm{~L}$ & Epilepsy & 197 \\
\hline H16.06.009 & $\mathrm{N}$ & 48 & $\mathrm{~F}$ & Caucasian & $\mathrm{N} / \mathrm{A}$ & $\mathrm{N} / \mathrm{A}$ & 7.1 & L & Epilepsy & 220 \\
\hline H16.03.004 & $\mathrm{N}$ & 25 & $M$ & Not noted & $\mathrm{N} / \mathrm{A}$ & $\mathrm{N} / \mathrm{A}$ & $\begin{array}{l}8.2 \pm \\
0.8\end{array}$ & $\mathrm{R}$ & $\begin{array}{l}\text { Tumor } \\
\text { removal, } \\
\text { epilepsy }\end{array}$ & 208 \\
\hline
\end{tabular}

1618

1619 Extended Data Table 1. Summary of human tissue donor information. Tissue types - $P$, postmortem, $\mathrm{N}$ - neurosurgical. Cause of death - CV, cardiovascular, N/A, not applicable. PMI 1621 postmortem interval. RIN - RNA Integrity Number. 
bioRxiv preprint doi: https://doi.org/10.1101/384826; this version posted August 5, 2018. The copyright holder for this preprint (which was not certified by peer review) is the author/funder, who has granted bioRxiv a license to display the preprint in perpetuity. It is made available under aCC-BY-NC-ND 4.0 International license.

A
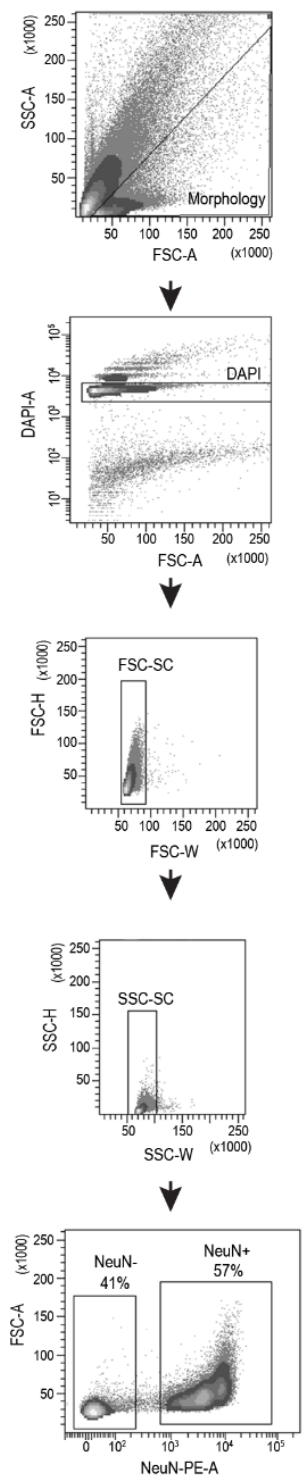

B

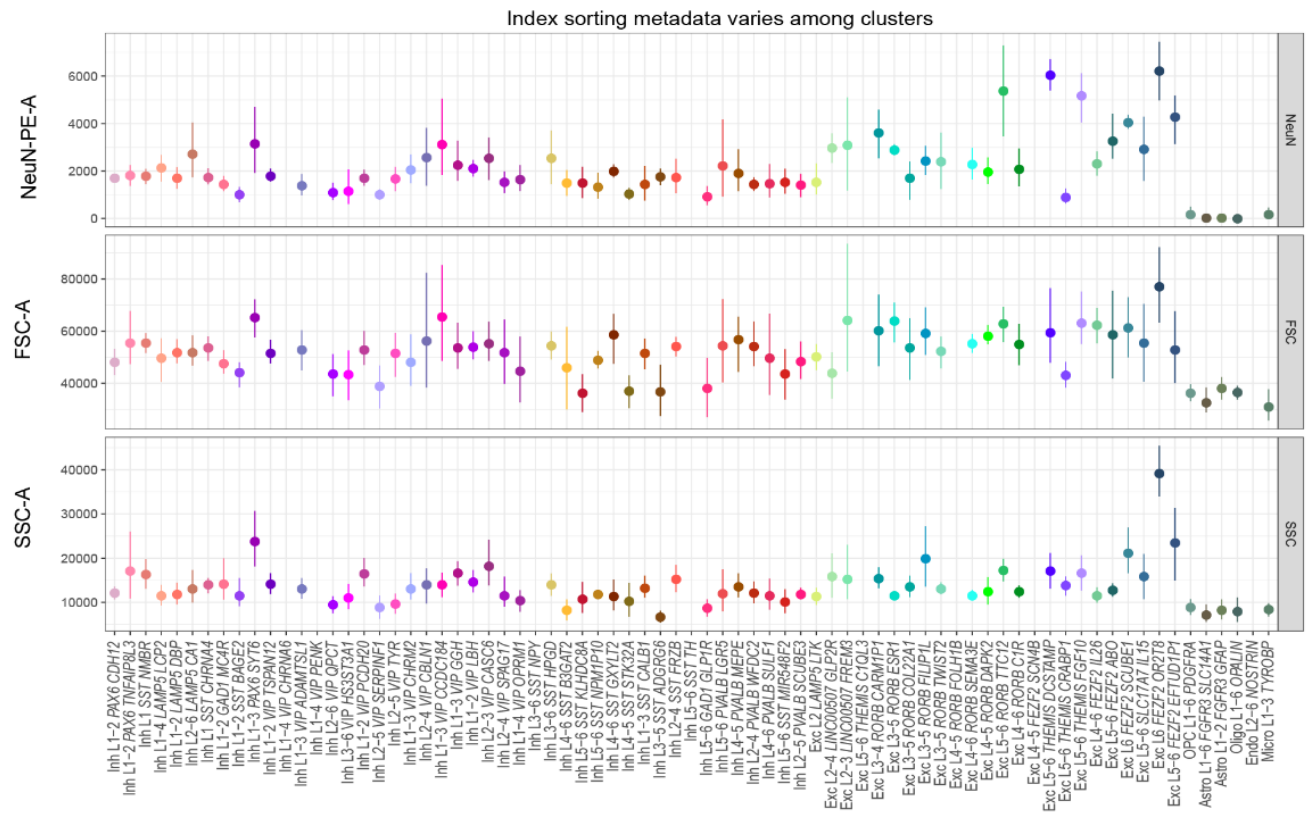

C

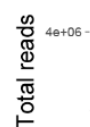

$20+06$

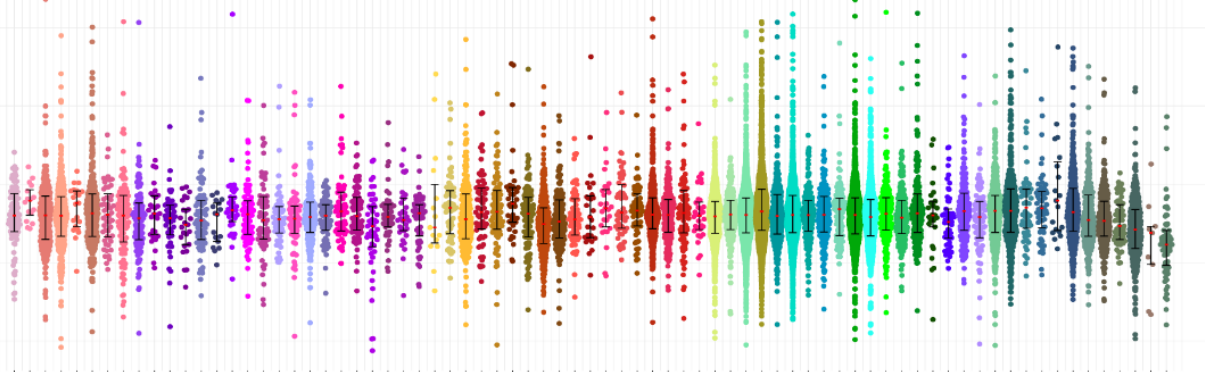

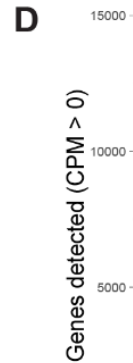

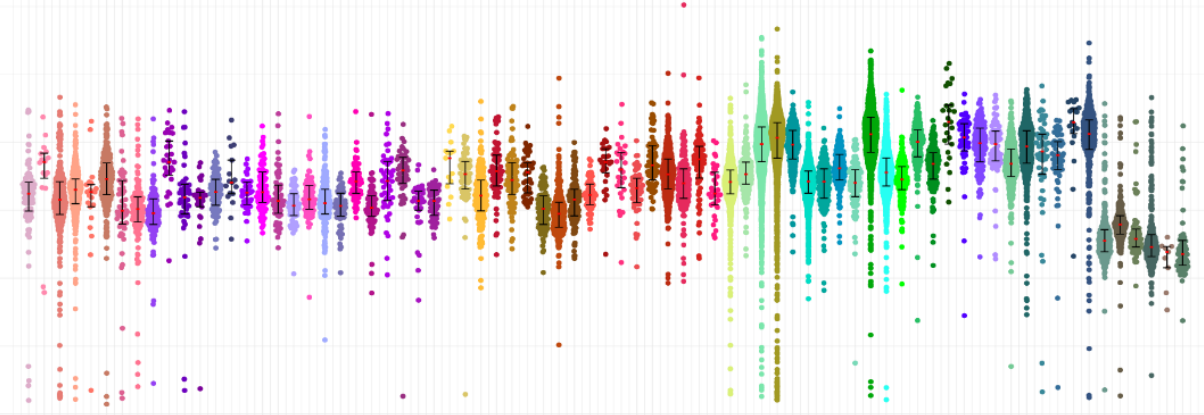

$\mathbf{E}$

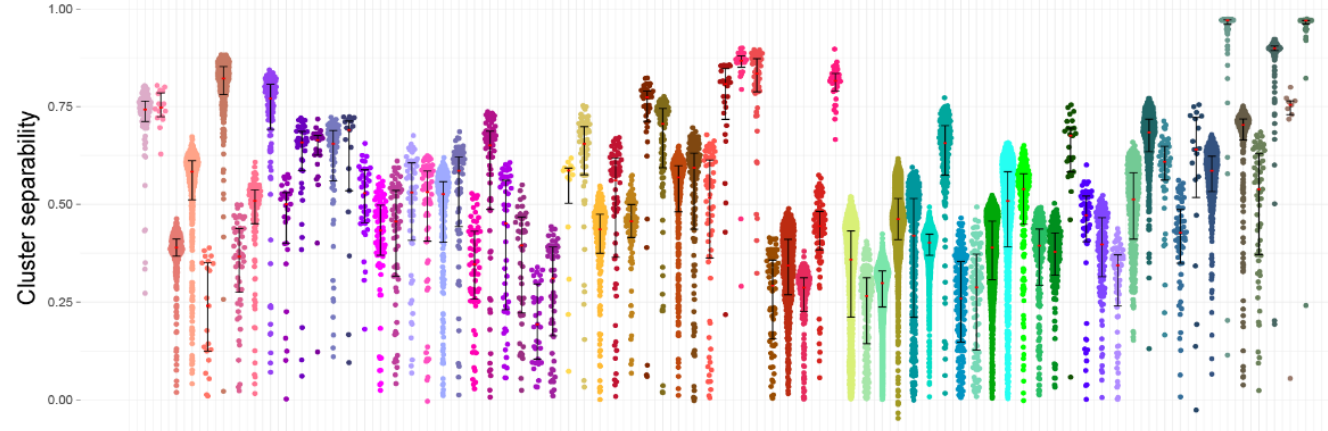


1623 Extended Data Figure 1. Nuclei metadata summarized by cluster. (A) FACS gating scheme for nuclei sorts. (B) FACS metadata for index sorted single nuclei shows significant variability in NeuN fluoresence intensity (NeuN-PE-A), size (forward-scatter area, FSC-A), and granularity (side-scatter area, SSC-A) across clusters. As expected, non-neuronal nuclei have almost no NeuN staining and are smaller (as inferred by lower FSC values). (C-E) Scatter plots plus median and interquartile interval of three QC metrics grouped and colored by cluster. (C) Median total reads were approximately 2.6 million for all cell types, although slightly lower for non-neuronal nuclei. (D) Median gene detection was highest among excitatory neuron types in layers 5 and 6 and a subset of types in layer 3, lower among inhibitory neuron types, and significantly lower among non-neuronal types. (E) Cluster separability varied substantially among cell types, with a subset of neuronal types and all non-neuronal types being highly

1634 discrete. 
bioRxiv preprint doi: https://doi org/10.1101/384826: this version posted August 5,2018 . The copyright holder for this preprint (which was not certified by peer review) is the author/funder, who has granted bioRxiv a license to display the preprint in perpetuity. It is made available under aCC-BY-NC-ND 4.0 International license.

A

Neurosurgical

\section{Postmortem}

B

Postmortem
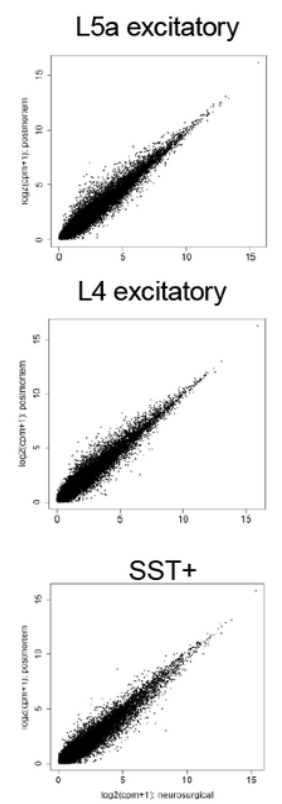

Neurosurgical
C

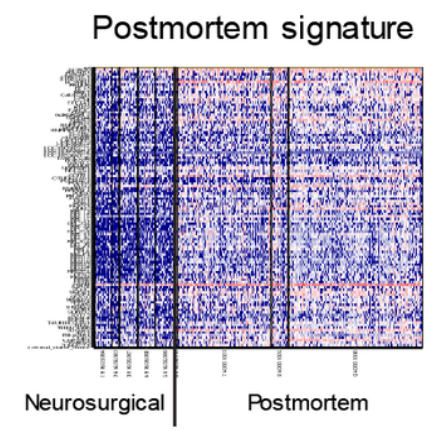

Includes quality

related genes

(ribosomal proteins)

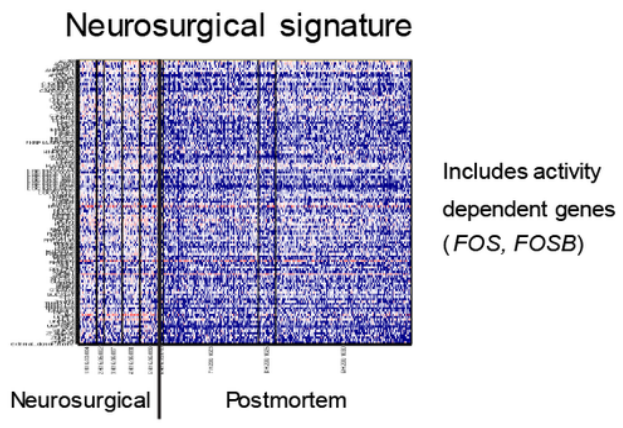


1636 Extended Data Figure 2. Small but consistent expression signature of donor tissue source. (A) Dot plot showing the proportion of nuclei isolated from neurosurgical and postmortem donors among human MTG clusters. Note that most nuclei from neurosurgical donors were isolated only from layer 5 so clusters enriched in other layers, such as layer 1 interneurons, have low representation of these donors. (B) Highly correlated expression between nuclei from postmortem and neurosurgical donors among two classes of excitatory neurons and one class of inhibitory neurons. Nuclei were pooled and compared within these broad classes due to the low sampling of individual clusters from neurosurgical donors. (C) Expression $\left(\log _{10}(C P M+1)\right)$ heatmaps of genes that are weakly but consistently upregulated in nuclei from postmortem or neurosurgical donors including ribosomal genes and activity-dependent genes, respectively. 
bioRxiv preprint doi: https://doi.org/10.1101/384826; this version posted August 5, 2018. The copyright holder for this preprint (which was not certified by peer review) is the author/funder, who has granted bioRxiv a license to display the preprint in perpetuity. It is made available under aCC-BY-NC-ND 4.0 International license.

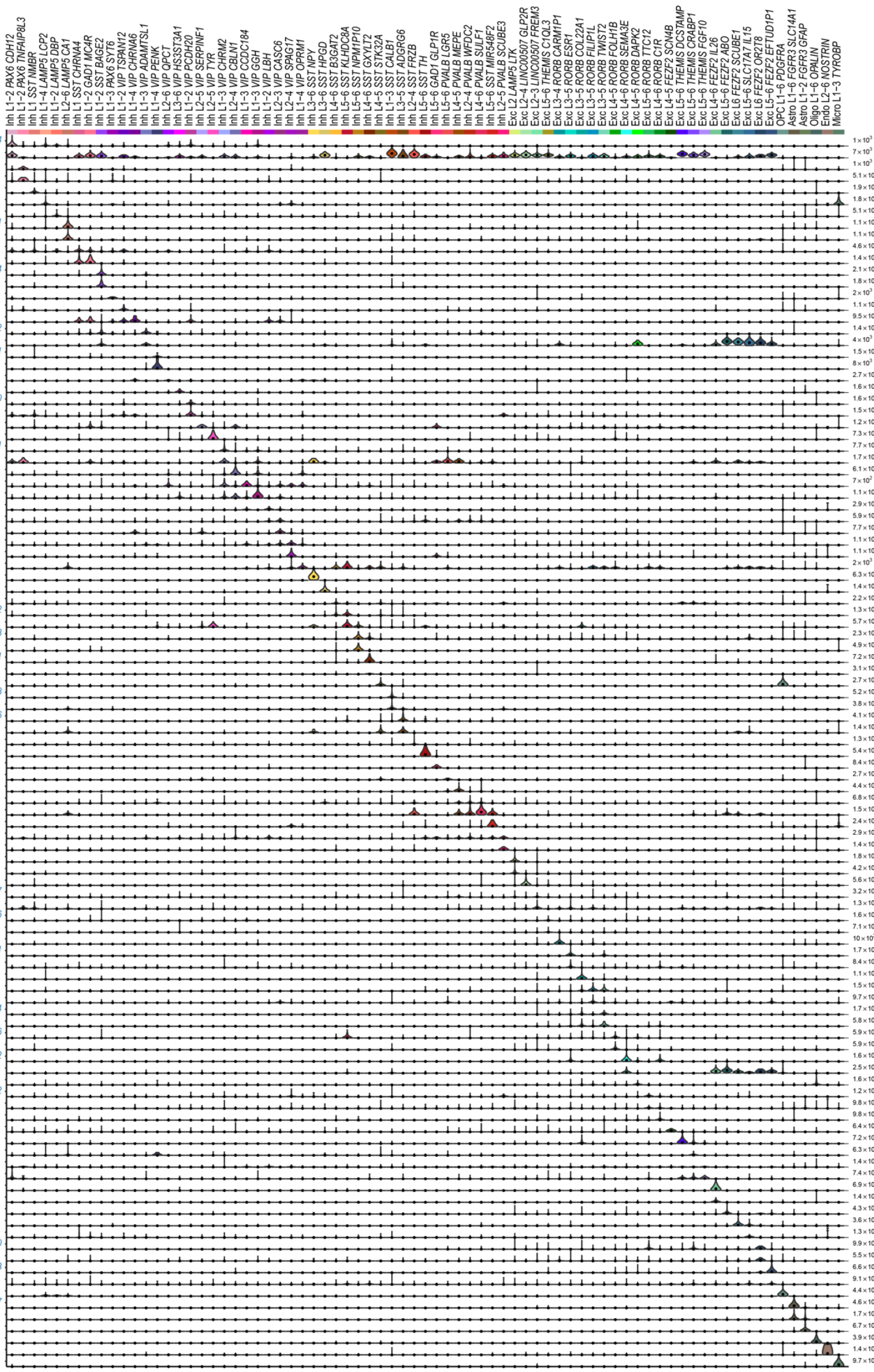


1648 Extended Data Figure 3. Expression of cell type specific markers. Violin plots of the best

1649 cell type markers include many non-coding genes (blue symbols): IncRNAs, antisense

1650 transcripts, and unnamed (LOC) genes. Expression values are on a linear scale and dots

1651 indicate median expression. Note that LOC genes were excluded from cluster names, and the best non-LOC marker genes were used instead. 


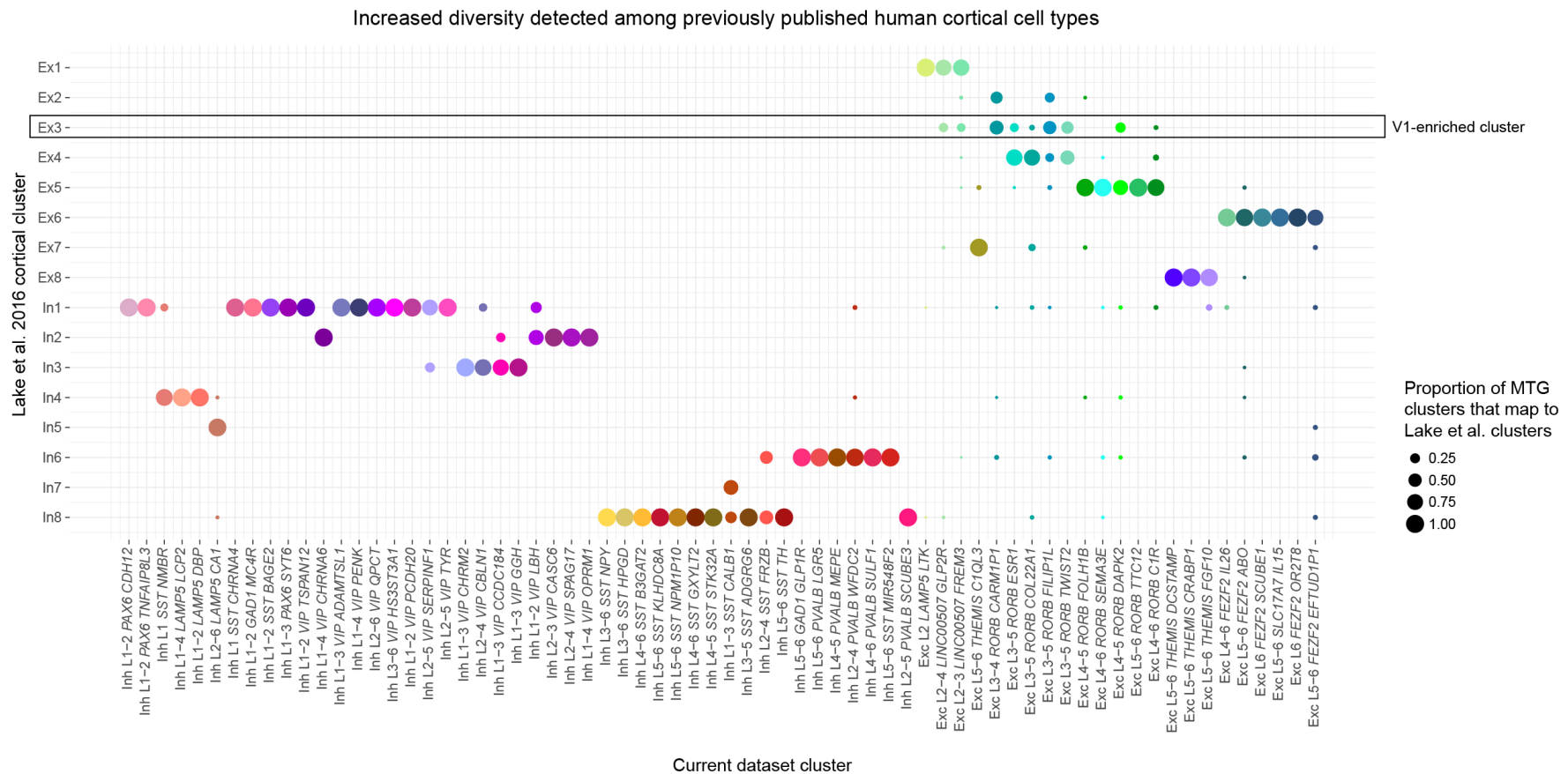

Extended Data Figure 4. Matching MTG clusters to reported human cortical cell types. Dot plot showing the proportion of each MTG cluster that matches 16 clusters reported by ${ }^{29}$ based on a centroid expression classifier. Ex3 was highly enriched in visual cortex and not detected in temporal cortex by Lake et al. 


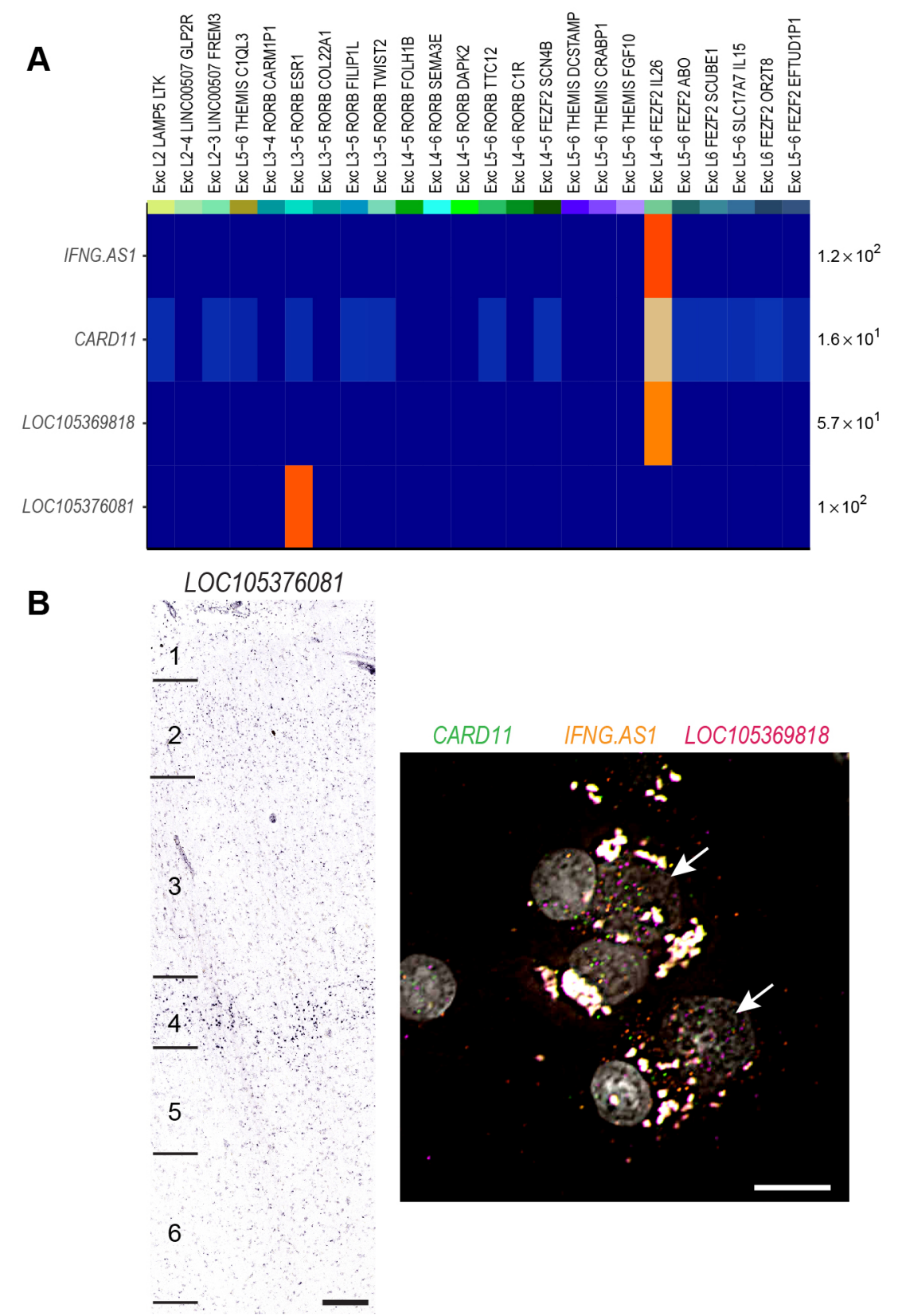

Extended Data Figure 5. In situ validation of LOC and antisense transcripts as cell type specific markers. (A) Heatmap illustrating cell type specific expression of several LOC genes and one antisense transcript (IFNG-AS1). (B) Left - chromogenic in situ hybridization for LOC105376081, a specific marker of the Exc L3-5 RORB ESR1 type shows expression of this gene predominantly in layer 4 , consistent with the anatomical location of this cell type. Scale bar, 100um. Right - triple RNAscope FISH for markers of the Exc L4-6 FEZF2 IL26 type. Coexpression of the protein coding gene CARD11 with IFNG-AS1, an antisense transcript, and LOC105369818 is apparent within several DAPI-labeled nuclei (white arrows). Scale bar, $166715 \mu \mathrm{m}$. 
A

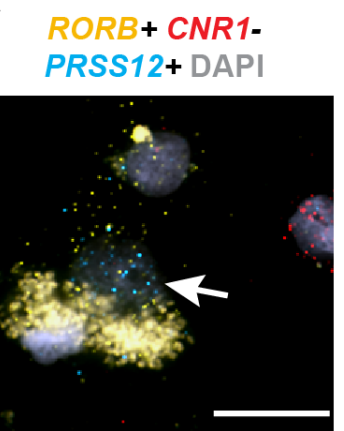

$$
\text { GRIK3+RORB+ }
$$
NTNG1- DAPI

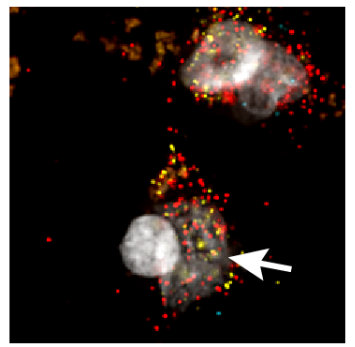

CRHR2+ RORB+ PRSS12- DAPI

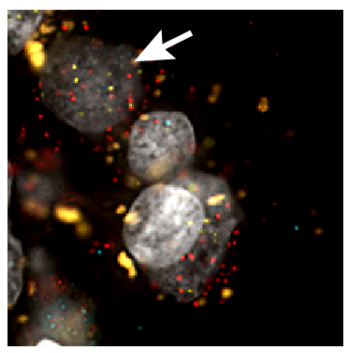

RORB+ MME+

NTNG1+ DAPI

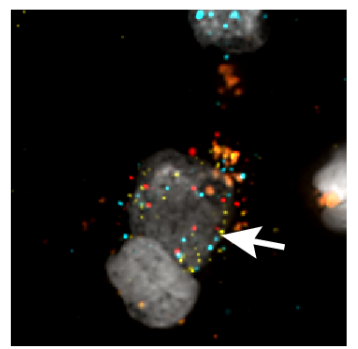

TPBG+ ALCAMMET- DAPI

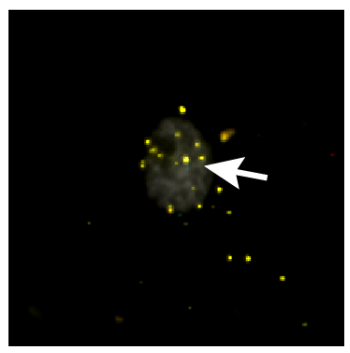

HS3ST4+ RORB+

CUX2+ DAPI

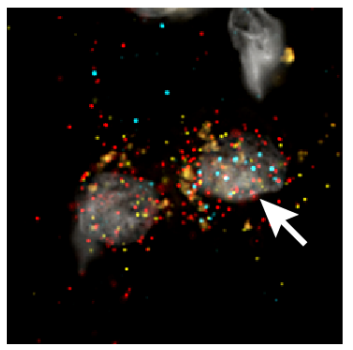

TPBG+ ALCAM+ MET- DAPI

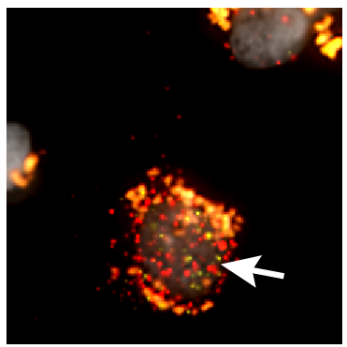

PCP4+ RORB+

GRIN3A+ DAPI

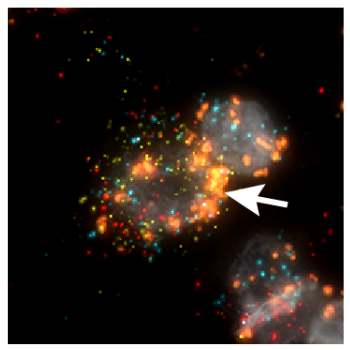

SLC17A7+ SMYD1+ POSTN+ DAPI

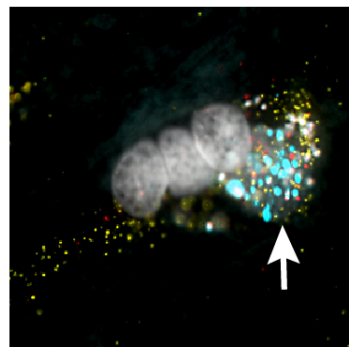

1669 Extended Data Figure 6. RNAscope multiplex FISH validation of 10 excitatory neuron

1670 types. Gene combinations probed are listed above each image. Labeled cells are indicated by 1671 white arrows. Scale bar, $20 \mu \mathrm{m}$. 

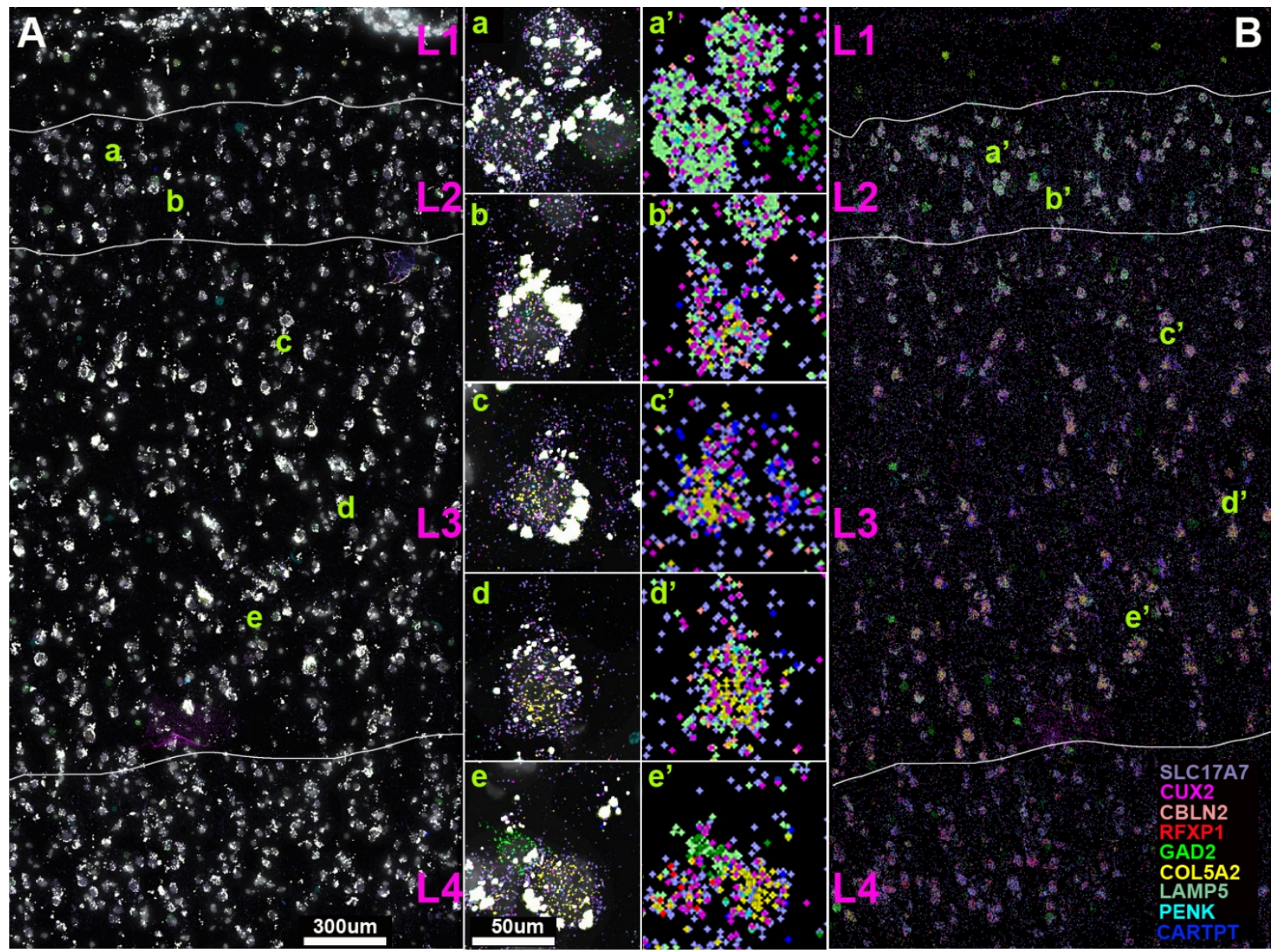

1673 Extended Data Figure 7. Single molecule (sm)FISH analysis of gene expression levels in

1674 human MTG layers 2 and 3. smFISH was performed with probes against SLC17A7, CUX2,

1675 CBLN2, RFXP1, GAD2, COL5A2, LAMP5, PENK, and CARTPT mRNA. (A) smFISH image (100x). Spots for each gene are pseudocolored as indicated in the bottom right legend. Layer demarcations are indicated in magenta. Scale bar $=300 \mathrm{um} . \mathrm{B})$ Spot indications for each gene, pseudocolored as indicated in the bottom right legend, as in A. a,a') Superficial layer 2 cells express SLC17A7(lavender), CUX2 (magenta), and LAMP5 (mint). b,b') At deeper locations in layer 2, an example of an SLC17A7-expressing cell with CUX2, LAMP5 and COL5A2

1681 expression. Note that LAMP5 expression (mint) decreases in CUX2/SLC17A7-expressing cells, while COL5A2/CUX2-expressing cells increase with depth along Layers 2 and 3 (see, c,c'; d,d';

1683 e,e'). 
bioRxiv preprint doi: https://doi.org/10.1101/384826; this version posted August 5, 2018. The copyright holder for this preprint (which was not certified by peer review) is the author/funder, who has granted bioRxiv a license to display the preprint in perpetuity. It is made available under aCC-BY-NC-ND 4.0 International license.

A In situ gene expression (smFISH probes)
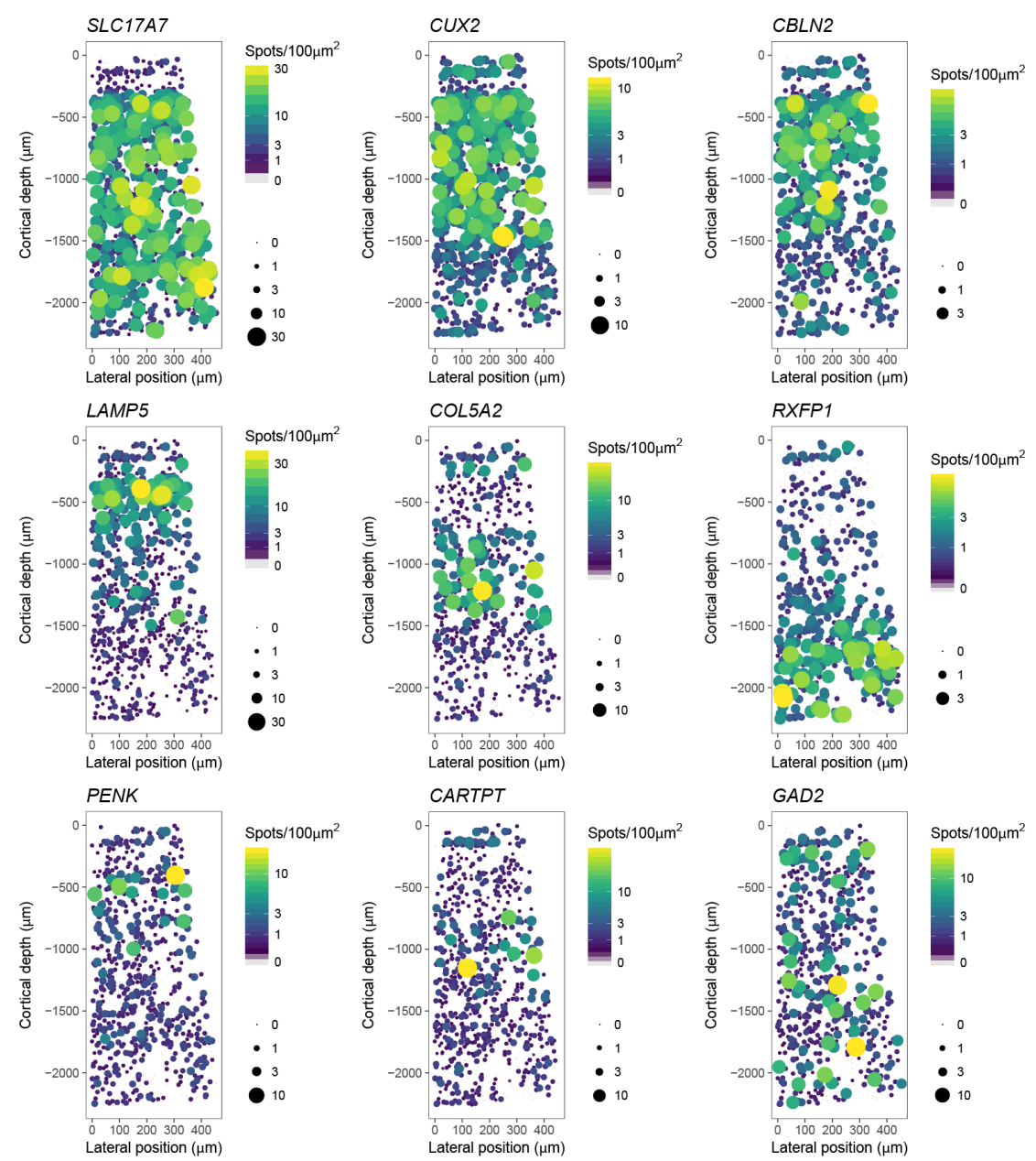

B

Spatial distribution of mapped glutamatergic cell types and classes
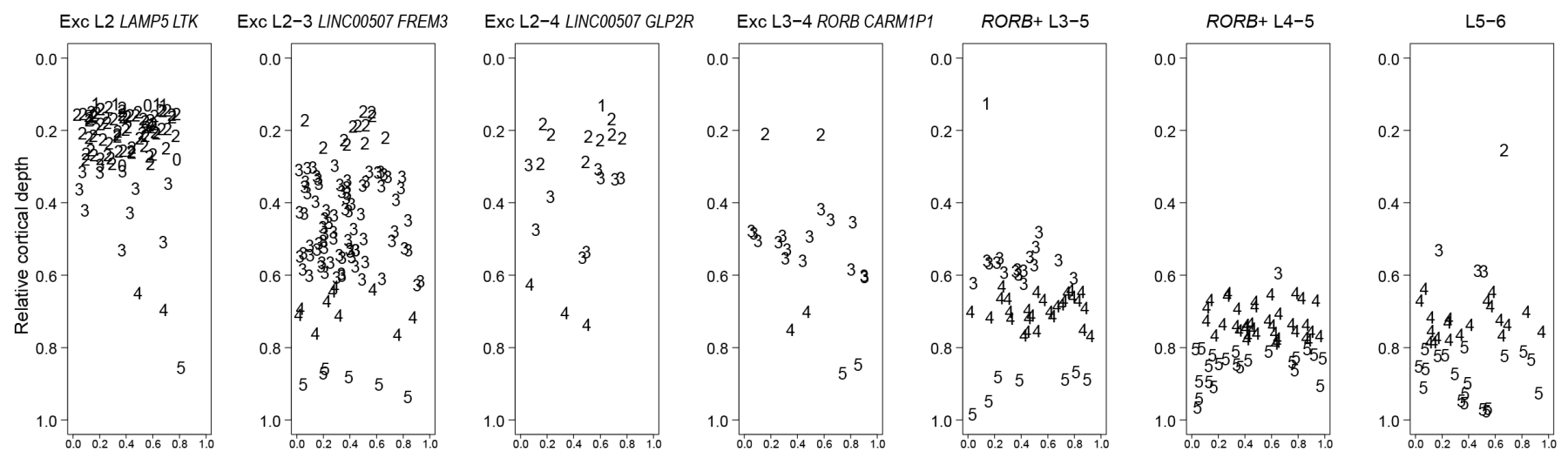

Relative lateral position 
1686 Extended Data Figure 8. Laminar distribution of superficial excitatory neuron types

1687 validated by smFISH. (A) Probe density (spots per 100 $\mu \mathrm{m} 2$ ) for 9 genes assayed across layers

1688 1-4 (and partially layer 5) of human MTG. The cortical slice was approximately $0.5 \mathrm{~mm}$ wide and

$16892 \mathrm{~mm}$ deep. Points correspond to cellular locations in situ where the y-axis is the cortical depth

1690 from the pial surface and the $x$-axis is the lateral position. Point size and color correspond to

1691 probe density. Cells that lack probe expression are shown as small grey points. (B) In situ

1692 location of cells mapped to indicated cell types and classes (different panels) based on

1693 expression levels of 9 genes shown in (A). Numbers indicate qualitative calls of the layer to

1694 which each cell belongs based on cytoarchitecture. 0 indicates that the cell was not annotated. 
bioRxiv preprint doi: https://doi.org/10.1101/384826: this version posted Auqust 5, 2018. The copyright holder for this preprint (which was not certified by peer review) is the author/funder, who has granted bioRxiv a license to display the preprint in perpetuity. It is made available under aCC-BY-NC-ND 4.0 International license.

A

Similar functional gene classes differentiate neuronal cell types but less so non-neuronal cell types in human and mouse
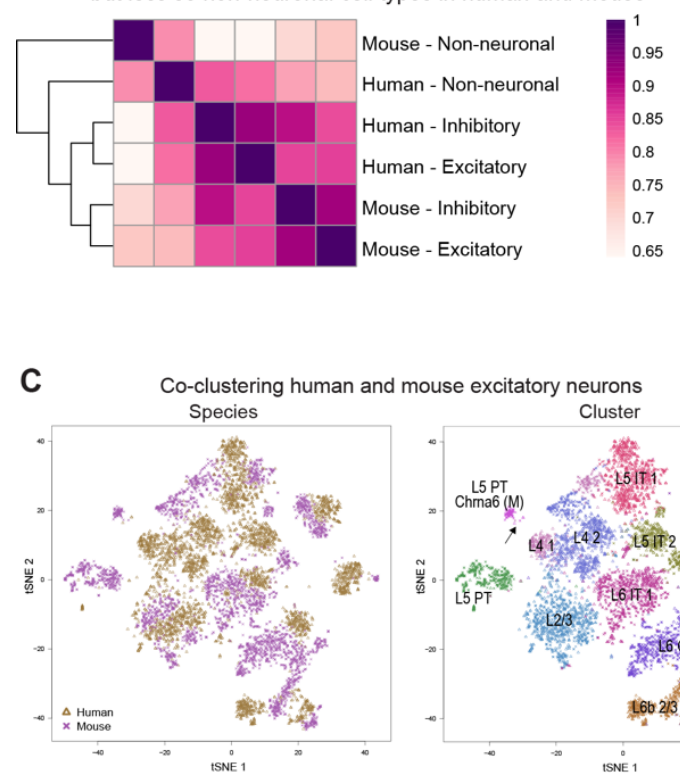

E

Inhibitory neurons

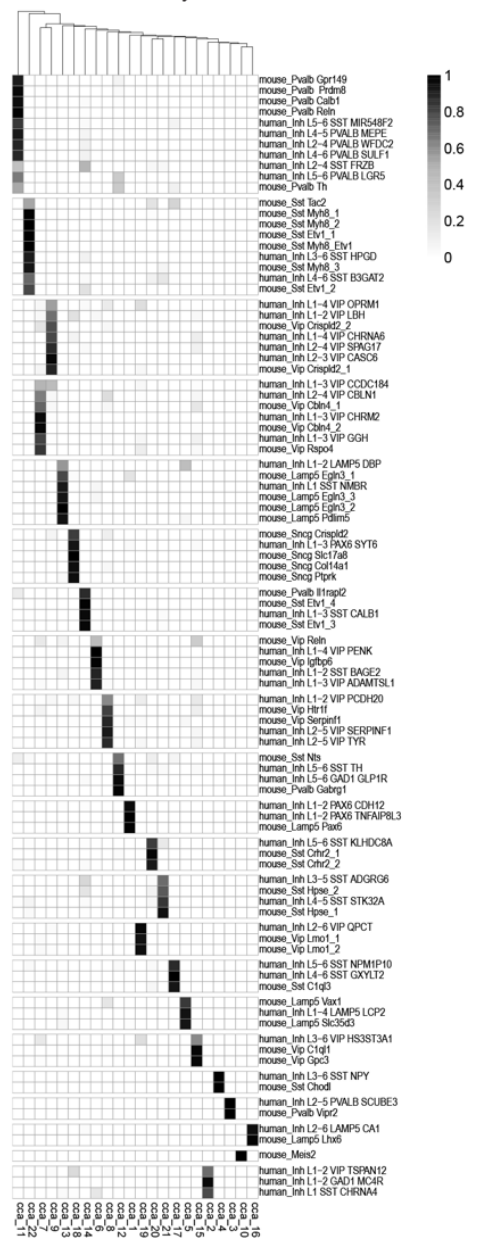

B

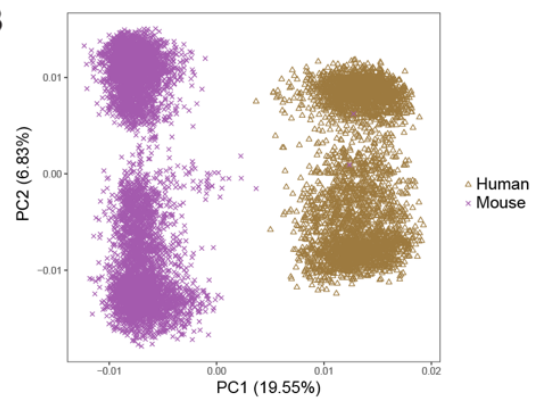

D Co-clustering human and mouse non-neuronal cells

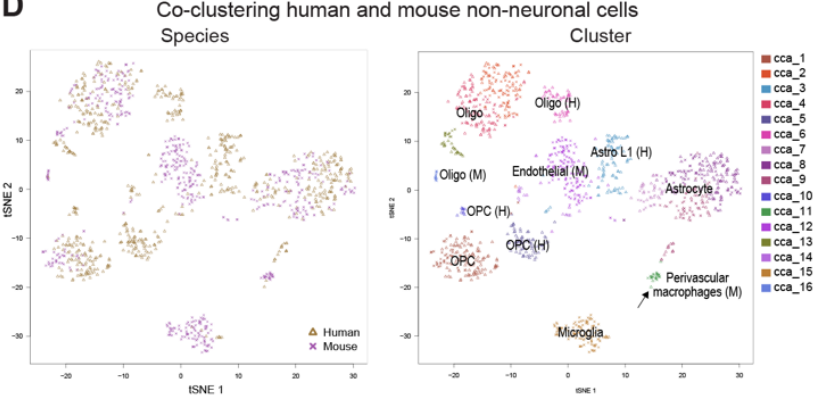

G

$\mathbf{F}$

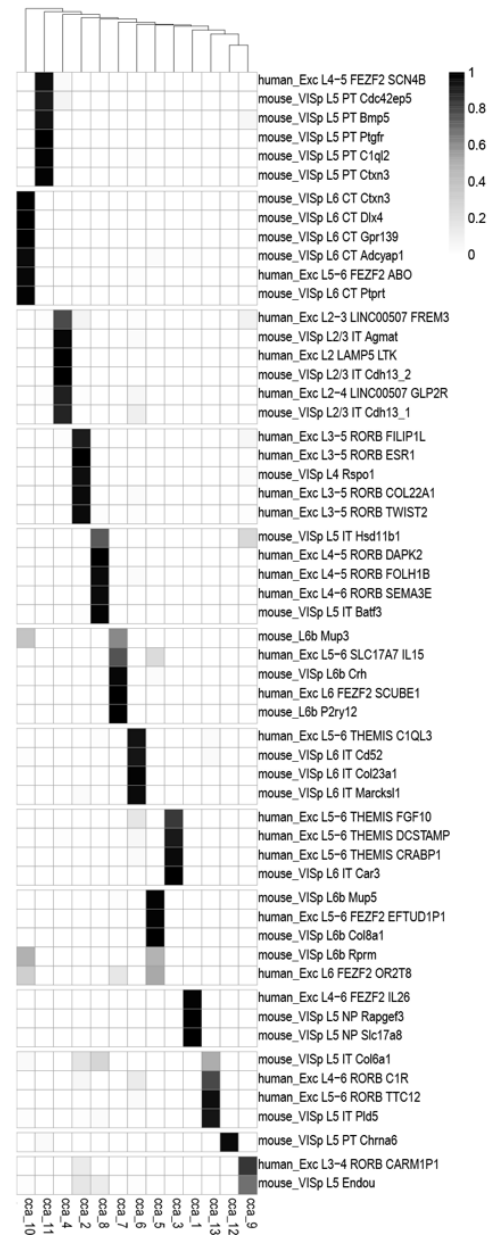

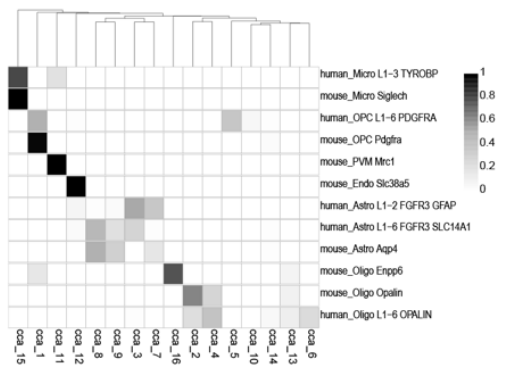
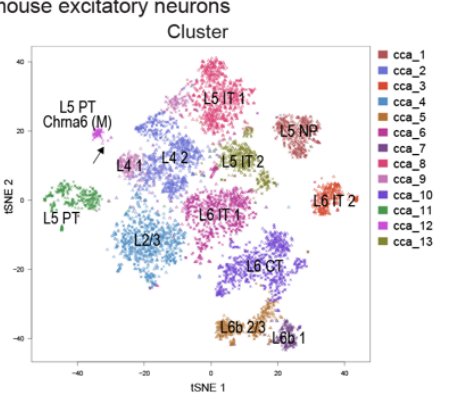
1696 Extended Data Figure 9. Quantifying human and mouse cell type homology. (A) Heatmap of Pearson's correlations between average MetaNeighbor AUROC scores for three broad classes of human and mouse cortical cell types. Rows and columns are ordered by averagelinkage hierarchical clustering. (B) Human (gold) and mouse (purple) GABAergic neurons projected on the first two principal components of a PCA combining expression data from both species. Almost $20 \%$ of expression differences are explained by species, while $6 \%$ are explained by major subclasses of interneurons. (C) t-SNE plots of first 30 basis vectors from a CCA of human and mouse glutamatergic neurons colored by species and CCA Cluster labeled with (M) contains only mouse cells. cluster. Arrow highlights two human nuclei that cluster with the mouse-specific (M) L5 PT Chrna6 cluster. (D) t-SNE plots of first 10 basis vectors from a CCA of human and mouse non-neuronal cells colored by species and CCA Clusters labeled with $(\mathrm{M})$ or $(\mathrm{H})$ contain only mouse cells or human nuclei, respectively. cluster. Human-specific $(\mathrm{H})$ and mouse-specific $(\mathrm{M})$ clusters are labeled. Arrow highlights two human nuclei that cluster with mouse perivascular macrophages. (E-G) Heatmaps showing the proportion of each human and mouse cluster (rows) that are members of each CCA cluster (columns) for GABAergic neurons (E), glutamatergic neurons $(F)$, and non-neuronal cells $(G)$. Rows and columns are hierarchically clustered, and most CCA clusters include human and mouse clusters that allows inference of homology between these clusters. 
bioRxiv preprint doi: https://doi.org/10.1101/384826; this version posted August 5, 2018. The copyright holder for this preprint (which was not certified by peer review) is the author/funder, who has granted bioRxiv a license to display the preprint in perpetuity. It is made available under aCC-BY-NC-ND 4.0 International license.

A

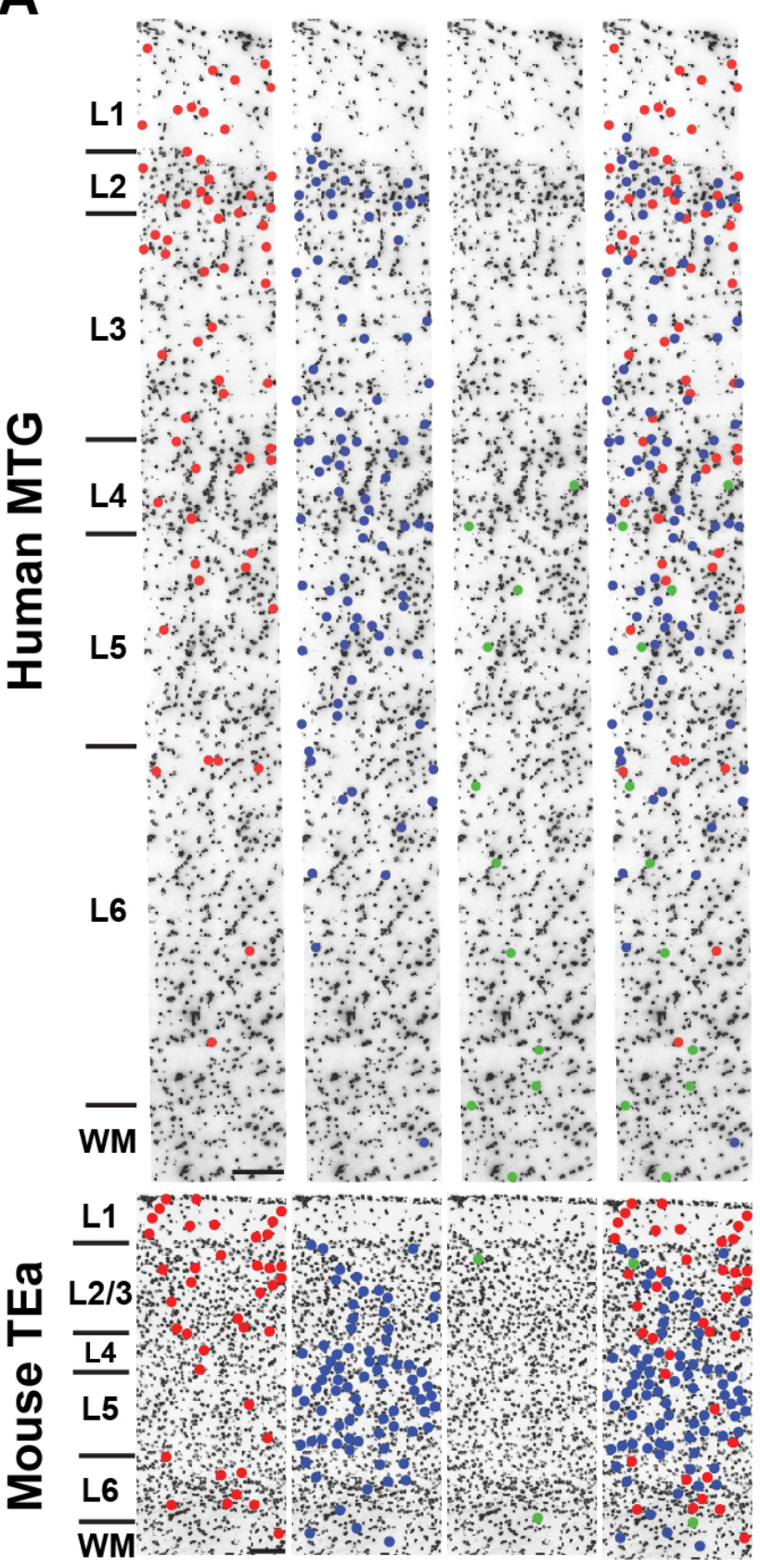

GAD1+/ADARB2+/LHX6-

GAD1+/ADARB2-/LHX6+

GAD1+/ADARB2+/LHX6+

GAD1 ADARB2 LHX6 DAPI

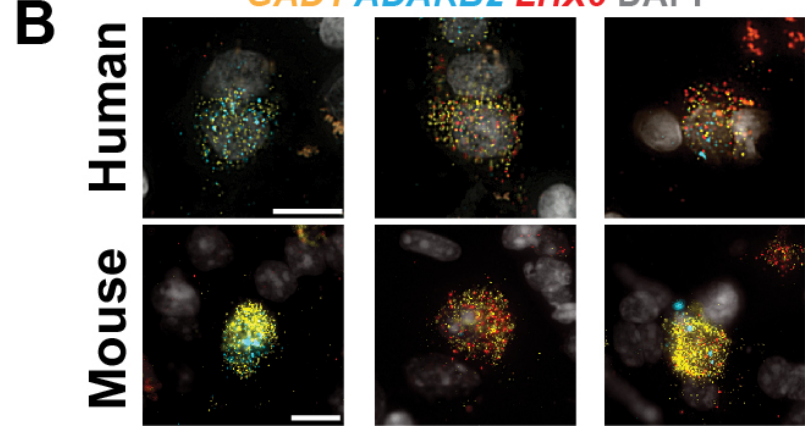


1716 Extended Data Figure 10. RNAscope multiplex FISH staining for broad interneuron class 1717 markers in human MTG and mouse temporal association area (TEa). (A) A representative

1718 inverted DAPI-stained cortical column illustrating the laminar positions of cells

1719 combinatorially labeled with broad interneuron class markers. Human MTG is shown in

1720 the top panels and mouse TEa is shown in the bottom panels. Left to right: red dots

1721 mark cells that are GAD1+/Gad1+, ADARB2+/Adarb2+, and LHX6-/Lhx6- (i.e. ADARB2

1722 branch interneurons); blue dots mark cells that are GAD1+/Gad1+, ADARB2-/Adarb2-,

1723 and $L H X 6+/ L h \times 6+$ (i.e. $L H X 6$ branch interneurons); green dots mark cells that

1724 are GAD1+/Gad1+, ADARB2+/Adarb2+, LHX6+/Lhx6+ (i.e. Inh L2-6 LAMP5 CA1 cells

1725 [human] or Lamp5 Lhx6 cells [mouse]); the far-right panel shows all the labeled cell

1726 classes overlaid onto the cortical column. (B) Representative images of cells labeled

1727 with the GAD1, ADARB2, and $L H X 6$ gene panel for human (top) and mouse (bottom).

1728 Left to right: cells double positive for $G A D 1$ and $A D A R B 2$; cells double positive

1729 for GAD1 and $L H X 6$; GAD1, ADARB2, and $L H X 6$ triple positive cells. Scale bars, $15 \mu \mathrm{m}$

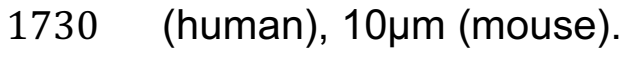


A

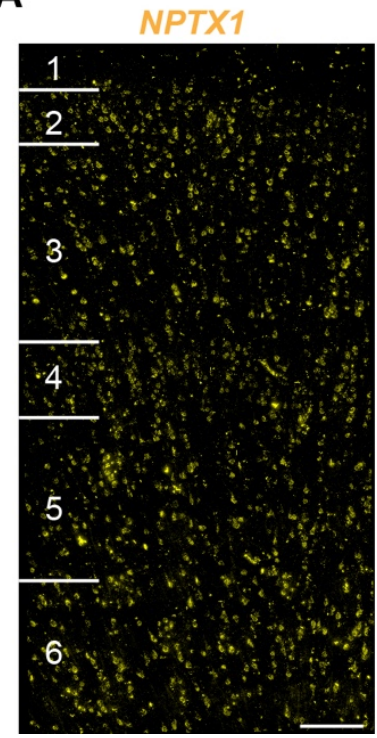

SLC17A7

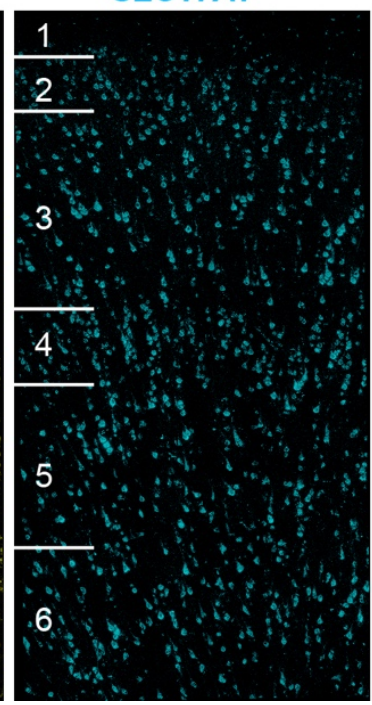

NPTX1 SLC17A7

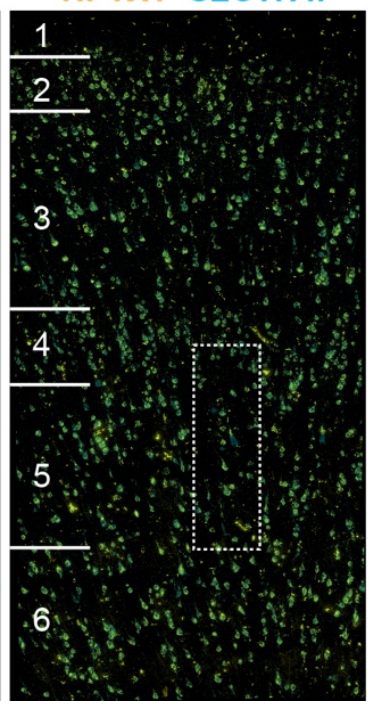

B

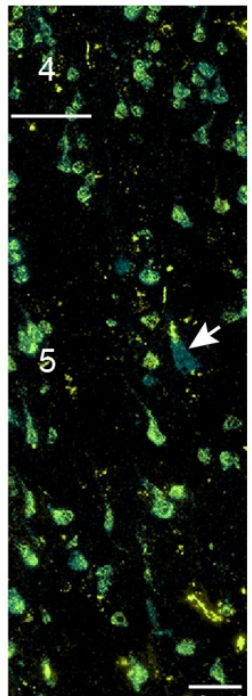

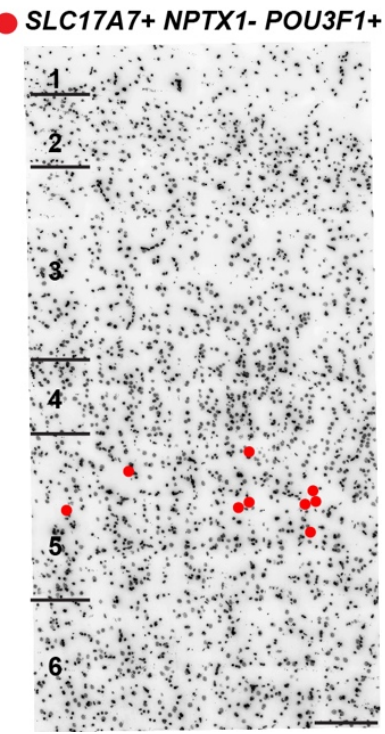

1732 Extended Data Figure 11. RNAscope multiplex FISH for markers of putative pyramidal tract (PT) neurons in human MTG. (A) FISH for NPTX1, a marker of non-PT excitatory types and SLC17A7, which is expressed in all excitatory neurons, shows that NPTX1 labels most SLC17A7+ cells across all cortical layers. The area indicated by the boxed region in the overlaid image of NPTX1 and SCL17A7 staining is shown at higher magnification in the adjacent panel to the right. One SLC17A7+ cell, indicated with the white arrow, is NPTX1-, but the rest of the SLC17A7+ cells in the field of view are NPTX1+. Scale bars, left (200um), right (50um). (B) A Representative inverted DAPI-stained cortical column overlaid with red dots that represent SLC17A7+, NPTX1-, and POU3F1+ cells. POU3F1 is a specific marker of the putative PT type (Exc L4-5 FEZF2 SCN4B). Scale bar, 200um. 


\section{Supplementary Data}

1743 Semantic representation of cell type clusters. To provide for an unambiguous, rigorous, and informative semantic representation of the cell types defined using the single nucleus RNA sequencing gene expression clusters, we developed a strategy for defining provisional cell types (pCL) in an rdf representation. We previously proposed that these provisional cell types could be defined using a combination of the tissue anatomic structure from which the assayed

1748 specimen was derived (e.g. middle temporal gyrus - go_sc:part_of uberon:UBERON_0002771), a set of marker genes whose combination is uniquely expressed in cells of the type (e.g.

1750

1751

1752

1753

1754

1755

1756

1757

1758

1759

1760

1761 mtg_cluster_sc:selectively_expresses hugo:HGNC_8620 and mtg_cluster_sc:selectively_expresses hugo:HGNC_1751), and a supertype parent class represented in either the $\mathrm{p} \bar{C} \mathrm{~L}$ or in the reference Cell Ontology (CL) (e.g. skos:broader :pCL78) [Bakken 2017, Aevermann 2018]. In addition, here we have also made the distinction between the experimental evidence supporting the existence of the provisional cell type class in the form of a single cell expression cluster ID (mtg_cluster_sc:evidence "Inh L1-2 PAX6 CDH12") and the provisional cell type class itself (e.g. pCL1). We also included additional knowledge about the location of instances of each provisional cell type class within specific layers of the cerebral cortex, both in terms of any layer in which the cell body of the type is found to be localized in (mtg_cluster_sc:has_soma_location_in) and in terms of the layer that a cell of the type is preferentially enriched in (mtg_cluster_sc:enriched_in). Finally, we included the cell cluster size (i.e. the number of single nuclei included in the cell type cluster from this particular experiment) as a rough estimate of the relative cell type abundance in the specimen assayed. This rdf representation is amenable to query-based inferencing using SPARQL.

Aevermann BD, Novotny M, Bakken T, Miller JA, Diehl AD, Osumi-Sutherland D, Lasken RS, Lein ES, Scheuermann RH. Cell type discovery using single-cell transcriptomics: implications for ontological representation. Hum Mol Genet. 2018 May 1;27(R1):R40-R47. doi:

1778 @prefix rdf: <http://www.w3.org/1999/02/22-rdf-syntax-ns\#> .

1779 @prefix rdfs: <http://www.w3.org/2000/01/rdf-schema\#>.

1780 @prefix xsd: <http://www.w3.org/2001/XMLSchema\#>.

1781 @prefix owl: <http://www.w3.org/2002/07/owl\#>. 
$1782 @ p r e f i x: @ \quad$ http://www.jcvi.org/cl ext/mtg cluster\#>.

$1783 @$ @prefix mtg_cluster_sc: <http://www.jcvi.org/cl ext/mtg cluster schema\#>.

1784 @prefix go_sc: <http://www.jcvi.org/cl ext/go schema\#>.

$1785 @$ @prefix cl: <http://purl.obolibrary.org/obo/cl\#>.

$1786 @$ @prefix hugo: <http://ncicb.nci.nih.gov/xml/owl/evs/hugo\#> .

1787 @prefix uberon: <http://purl.obolibrary.org/obo/uberon\#>.

1788 @prefix skos: <http://www.w3.org/2004/02/skos/core\#>.

1789

$1790 \quad<$ http://www.jcvi.org/cl ext/mtg cluster>

1791 rdf:type owl:Ontology ;

1792 owl:versionlnfo "\$ld: mtg_cluster_v4.ttl, Ver 1.0, May 23, 2018, Mohamed Keshk. \$" .

1793

1794

1795

$\#^{* * * * * * * * * * * * * * * *}$ MTG_Cluster_Annotation Concepts

1796

$1797: p C L 1$

1798 a skos:Concept ;

1799 mtg_cluster_sc:id "pCL1" ;

$1800 \quad$ skos:broader :pCL78;

1801 rdfs:label "PAX6|CDH12-expressing cerebral cortex MTG GABAergic interneuron" ;

1802 mtg_cluster_sc:evidence "Inh L1-2 PAX6 CDH12" ;

1803 go_sc:part_of uberon:UBERON_0002771;

1804 mtg_cluster_sc:enriched_in "cortical_layer1" ;

1805 mtg_cluster_sc:has_soma_location_in "cortical_layer1" ;

1806 mtg_cluster_sc:has_soma_location_in "cortical_layer2" ;

1807 mtg_cluster_sc:selectively_expresses hugo:HGNC_8620 ;

1808 mtg_cluster_sc:selectively_expresses hugo:HGNC_1751;

1809 mtg_cluster_sc:neuron_type "GABAergic" ;

1810 mtg_cluster_sc:cluster_size "90"^^xsd:int ; 
1812 :pCL2

1813 a skos:Concept ;

1814 mtg_cluster_sc:id "pCL2" ;

$1815 \quad$ skos:broader :pCL78 ;

1816 rdfs:label "PAX6|TNFAIP8L3-expressing cerebral cortex MTG GABAergic interneuron" ;

1817 mtg_cluster_sc:evidence "Inh L1-2 PAX6 TNFAIP8L3" ;

1818 go_sc:part_of uberon:UBERON_0002771;

1819 mtg_cluster_sc:enriched_in "cortical_layer2" ;

1820 mtg_cluster_sc:has_soma_location_in "cortical_layer1" ;

1821 mtg_cluster_sc:has_soma_location_in "cortical_layer2" ;

1822 mtg_cluster_sc:selectively_expresses hugo:HGNC_8620 ;

1823 mtg_cluster_sc:selectively_expresses hugo:HGNC_20620 ;

1824 mtg_cluster_sc:neuron_type "GABAergic" ;

1825 mtg_cluster_sc:cluster_size "16"^^xsd:int ;

1826.

1827 :pCL3

1828 a skos:Concept ;

1829 mtg_cluster_sc:id "pCL3" ;

1830 skos:broader :pCL78 ;

1831 rdfs:label "SST|NMBR-expressing cerebral cortex MTG GABAergic interneuron" ;

1832 mtg_cluster_sc:evidence "Inh L1 SST NMBR" ;

1833 go_sc:part_of uberon:UBERON_0002771 ;

1834 mtg_cluster_sc:enriched_in "cortical_layer1" ;

1835 mtg_cluster_sc:has_soma_location_in "cortical_layer1" ;

1836 mtg_cluster_sc:selectively_expresses hugo:HGNC_11329 ;

1837 mtg_cluster_sc:selectively_expresses hugo:HGNC_7843;

1838 mtg_cluster_sc:neuron_type "GABAergic" ;

1839 mtg_cluster_sc:cluster_size "283"^^xsd:int ; 
1841 :pCL4

1842 a skos:Concept ;

1843 mtg_cluster_sc:id "pCL4" ;

1844 skos:broader :pCL78 ;

1845 rdfs:label "LAMP5|LCP2-expressing cerebral cortex MTG GABAergic interneuron" ;

1846 mtg_cluster_sc:evidence "Inh L1-4 LAMP5 LCP2" ;

1847 go_sc:part_of uberon:UBERON_0002771;

1848 mtg_cluster_sc:enriched_in "cortical_layer3" ;

1849 mtg_cluster_sc:has_soma_location_in "cortical_layer1" ;

1850 mtg_cluster_sc:has_soma_location_in "cortical_layer2" ;

1851 mtg_cluster_sc:has_soma_location_in "cortical_layer3" ;

1852 mtg_cluster_sc:has_soma_location_in "cortical_layer4" ;

1853 mtg_cluster_sc:selectively_expresses hugo:HGNC_16097 ;

1854 mtg_cluster_sc:selectively_expresses hugo:HGNC_6529 ;

1855 mtg_cluster_sc:neuron_type "GABAergic" ;

1856 mtg_cluster_sc:cluster_size "356"^^xsd:int ;

1857

1858 :pCL5

1859 a skos:Concept ;

1860 mtg_cluster_sc:id "pCL5" ;

1861 skos:broader :pCL78 ;

1862 rdfs:label "LAMP5|DBP-expressing cerebral cortex MTG GABAergic interneuron" ;

1863 mtg_cluster_sc:evidence "Inh L1-2 LAMP5 DBP" ;

1864 go_sc:part_of uberon:UBERON_0002771 ;

1865 mtg_cluster_sc:enriched_in "cortical_layer1" ;

1866 mtg_cluster_sc:has_soma_location_in "cortical_layer1" ;

1867 mtg_cluster_sc:has_soma_location_in "cortical_layer2" ;

1868 mtg_cluster_sc:selectively_expresses hugo:HGNC_16097 ; 
1869 mtg_cluster_sc:selectively_expresses hugo:HGNC_2697;

1870 mtg_cluster_sc:neuron_type "GABAergic" ;

1871 mtg_cluster_sc:cluster_size "21"^^xsd:int ;

1872

1873 :pCL6

1874 a skos:Concept ;

1875 mtg_cluster_sc:id "pCL6" ;

1876 skos:broader :pCL78 ;

1877 rdfs:label "LAMP5|CA1-expressing cerebral cortex MTG GABAergic interneuron" ;

1878 mtg_cluster_sc:evidence "Inh L2-6 LAMP5 CA1" ;

1879 go_sc:part_of uberon:UBERON_0002771 ;

1880 mtg_cluster_sc:enriched_in "cortical_layer5" ;

1881 mtg_cluster_sc:has_soma_location_in "cortical_layer2" ;

1882 mtg_cluster_sc:has_soma_location_in "cortical_layer3" ;

1883 mtg_cluster_sc:has_soma_location_in "cortical_layer4" ;

1884 mtg_cluster_sc:has_soma_location_in "cortical_layer5" ;

1885 mtg_cluster_sc:has_soma_location_in "cortical_layer6" ;

1886 mtg_cluster_sc:selectively_expresses hugo:HGNC_16097 ;

1887 mtg_cluster_sc:selectively_expresses hugo:HGNC_1368 ;

1888 mtg_cluster_sc:neuron_type "GABAergic" ;

1889 mtg_cluster_sc:cluster_size "256"^^xsd:int ;

1890

$1891:$ :pCL7

1892 a skos:Concept ;

1893 mtg_cluster_sc:id "pCL7" ;

1894 skos:broader :pCL78;

1895 rdfs:label "SST|CHRNA4-expressing cerebral cortex MTG GABAergic interneuron" ;

1896 mtg_cluster_sc:evidence "Inh L1 SST CHRNA4" ;

1897 go_sc:part_of uberon:UBERON_0002771 ; 
1898 mtg_cluster_sc:enriched_in "cortical_layer1" ;

1899 mtg_cluster_sc:has_soma_location_in "cortical_layer1" ;

1900 mtg_cluster_sc:selectively_expresses hugo:HGNC_11329;

1901 mtg_cluster_sc:selectively_expresses hugo:HGNC_1958;

1902 mtg_cluster_sc:neuron_type "GABAergic" ;

1903 mtg_cluster_sc:cluster_size "52"^^xsd:int ;

1904

1905 :pCL8

1906 a skos:Concept ;

1907 mtg_cluster_sc:id "pCL8" ;

1908 skos:broader :pCL78;

1909 rdfs:label "GAD1|MC4R-expressing cerebral cortex MTG GABAergic interneuron" ;

1910 mtg_cluster_sc:evidence "Inh L1-2 GAD1 MC4R" ;

1911 go_sc:part_of uberon:UBERON_0002771 ;

1912 mtg_cluster_sc:enriched_in "cortical_layer1" ;

1913 mtg_cluster_sc:has_soma_location_in "cortical_layer1" ;

1914 mtg_cluster_sc:has_soma_location_in "cortical_layer2" ;

1915 mtg_cluster_sc:selectively_expresses hugo:HGNC_4092;

1916 mtg_cluster_sc:selectively_expresses hugo:HGNC_6932 ;

1917 mtg_cluster_sc:neuron_type "GABAergic" ;

1918 mtg_cluster_sc:cluster_size "107"^^xsd:int ;

1919

1920 :pCL9

1921 a skos:Concept ;

1922 mtg_cluster_sc:id "pCL9" ;

1923 skos:broader :pCL78;

1924 rdfs:label "SST|BAGE2-expressing cerebral cortex MTG GABAergic interneuron" ;

1925 mtg_cluster_sc:evidence "Inh L1-2 SST BAGE2" ;

1926 go_sc:part_of uberon:UBERON_0002771; 
1927 mtg_cluster_sc:enriched_in "cortical_layer1" ;

1928 mtg_cluster_sc:has_soma_location_in "cortical_layer1" ;

1929 mtg_cluster_sc:has_soma_location_in "cortical_layer2" ;

1930 mtg_cluster_sc:selectively_expresses hugo:HGNC_11329 ;

1931 mtg_cluster_sc:selectively_expresses hugo:HGNC_15723 ;

1932 mtg_cluster_sc:neuron_type "GABAergic" ;

1933 mtg_cluster_sc:cluster_size "108"^^xsd:int ;

1934

$1935:$ :pCL10

1936 a skos:Concept ;

1937 mtg_cluster_sc:id "pCL10" ;

1938 skos:broader :pCL80 ;

1939 rdfs:label "PAX6|SYT6-expressing cerebral cortex MTG GABAergic interneuron" ;

1940 mtg_cluster_sc:evidence "Inh L1-3 PAX6 SYT6" ;

1941 go_sc:part_of uberon:UBERON_0002771 ;

1942 mtg_cluster_sc:enriched_in "cortical_layer2" ;

1943 mtg_cluster_sc:has_soma_location_in "cortical_layer1" ;

1944 mtg_cluster_sc:has_soma_location_in "cortical_layer2" ;

1945 mtg_cluster_sc:has_soma_location_in "cortical_layer3" ;

1946 mtg_cluster_sc:selectively_expresses hugo:HGNC_8620 ;

1947 mtg_cluster_sc:selectively_expresses hugo:HGNC_18638 ;

1948 mtg_cluster_sc:neuron_type "GABAergic" ;

1949 mtg_cluster_sc:cluster_size "29"^^xsd:int ;

1950

$1951:$ :pCL11

1952 a skos:Concept ;

1953 mtg_cluster_sc:id "pCL11" ;

1954 skos:broader :pCL80 ;

1955 rdfs:label "TSPAN12-expressing cerebral cortex MTG GABAergic interneuron" ; 
1956 mtg_cluster_sc:evidence "Inh L1-2 VIP TSPAN12" ;

1957 go_sc:part_of uberon:UBERON_0002771 ;

1958 mtg_cluster_sc:enriched_in "cortical_layer1" ;

1959 mtg_cluster_sc:has_soma_location_in "cortical_layer1" ;

1960 mtg_cluster_sc:has_soma_location_in "cortical_layer2" ;

1961 mtg_cluster_sc:selectively_expresses hugo:HGNC_21641;

1962 mtg_cluster_sc:neuron_type "GABAergic" ;

1963 mtg_cluster_sc:cluster_size "42"^^xsd:int ;

1964.

$1965:$ :pCL12

1966 a skos:Concept ;

1967 mtg_cluster_sc:id "pCL12" ;

1968 skos:broader :pCL80 ;

1969 rdfs:label "CHRNA6-expressing cerebral cortex MTG GABAergic interneuron" ;

1970 mtg_cluster_sc:evidence "Inh L1-4 VIP CHRNA6" ;

1971 go_sc:part_of uberon:UBERON_0002771;

1972 mtg_cluster_sc:enriched_in "cortical_layer3" ;

1973 mtg_cluster_sc:has_soma_location_in "cortical_layer1" ;

1974 mtg_cluster_sc:has_soma_location_in "cortical_layer2" ;

1975 mtg_cluster_sc:has_soma_location_in "cortical_layer3" ;

1976 mtg_cluster_sc:has_soma_location_in "cortical_layer4" ;

1977 mtg_cluster_sc:selectively_expresses hugo:HGNC_15963 ;

1978 mtg_cluster_sc:neuron_type "GABAergic" ;

1979 mtg_cluster_sc:cluster_size "25"^^xsd:int ;

1980

1981 :pCL13

1982 a skos:Concept ;

1983 mtg_cluster_sc:id "pCL13" ;

1984 skos:broader :pCL80 ; 
1985 rdfs:label "ADAMTSL1-expressing cerebral cortex MTG GABAergic interneuron" ;

1986 mtg_cluster_sc:evidence "Inh L1-3 VIP ADAMTSL1" ;

1987 go_sc:part_of uberon:UBERON_0002771 ;

1988 mtg_cluster_sc:enriched_in "cortical_layer3" ;

1989 mtg_cluster_sc:has_soma_location_in "cortical_layer1" ;

1990 mtg_cluster_sc:has_soma_location_in "cortical_layer2" ;

1991 mtg_cluster_sc:has_soma_location_in "cortical_layer3" ;

1992 mtg_cluster_sc:selectively_expresses hugo:HGNC_14632 ;

1993 mtg_cluster_sc:neuron_type "GABAergic" ;

1994 mtg_cluster_sc:cluster_size "72"^^xsd:int ;

1995

$1996:$ :pL14

1997 a skos:Concept ;

1998 mtg_cluster_sc:id "pCL14" ;

1999 skos:broader :pCL80 ;

2000 rdfs:label "PENK-expressing cerebral cortex MTG GABAergic interneuron" ;

2001 mtg_cluster_sc:evidence "Inh L1-4 VIP PENK" ;

2002 go_sc:part_of uberon:UBERON_0002771;

2003 mtg_cluster_sc:enriched_in "cortical_layer3" ;

2004 mtg_cluster_sc:has_soma_location_in "cortical_layer1" ;

2005 mtg_cluster_sc:has_soma_location_in "cortical_layer2" ;

2006 mtg_cluster_sc:has_soma_location_in "cortical_layer3" ;

2007 mtg_cluster_sc:has_soma_location_in "cortical_layer4" ;

2008 mtg_cluster_sc:selectively_expresses hugo:HGNC_8831;

2009 mtg_cluster_sc:neuron_type "GABAergic" ;

2010 mtg_cluster_sc:cluster_size "17"^^xsd:int ;

2011

$2012:$ :pCL15

2013 a skos:Concept ; 
2014 mtg_cluster_sc:id "pCL15" ;

2015 skos:broader :pCL80 ;

2016 rdfs:label "QPCT-expressing cerebral cortex MTG GABAergic interneuron" ;

2017 mtg_cluster_sc:evidence "Inh L2-6 VIP QPCT" ;

2018 go_sc:part_of uberon:UBERON_0002771;

2019 mtg_cluster_sc:enriched_in "cortical_layer4" ;

2020 mtg_cluster_sc:has_soma_location_in "cortical_layer2" ;

2021 mtg_cluster_sc:has_soma_location_in "cortical_layer3" ;

2022 mtg_cluster_sc:has_soma_location_in "cortical_layer4" ;

2023 mtg_cluster_sc:has_soma_location_in "cortical_layer5" ;

2024 mtg_cluster_sc:has_soma_location_in "cortical_layer6" ;

2025 mtg_cluster_sc:selectively_expresses hugo:HGNC_9753 ;

2026 mtg_cluster_sc:neuron_type "GABAergic" ;

2027 mtg_cluster_sc:cluster_size "37"^^xsd:int ;

2028 .

$2029: p C L 16$

2030 a skos:Concept ;

2031 mtg_cluster_sc:id "pCL16" ;

2032 skos:broader :pCL80;

2033 rdfs:label "HS3ST3A1-expressing cerebral cortex MTG GABAergic interneuron" ;

2034 mtg_cluster_sc:evidence "Inh L3-6 VIP HS3ST3A1" ;

2035 go_sc:part_of uberon:UBERON_0002771;

2036 mtg_cluster_sc:enriched_in "cortical_layer4" ;

2037 mtg_cluster_sc:has_soma_location_in "cortical_layer3" ;

2038 mtg_cluster_sc:has_soma_location_in "cortical_layer4" ;

2039 mtg_cluster_sc:has_soma_location_in "cortical_layer5" ;

2040 mtg_cluster_sc:has_soma_location_in "cortical_layer6" ;

2041 mtg_cluster_sc:selectively_expresses hugo:HGNC_5196 ;

2042 mtg_cluster_sc:neuron_type "GABAergic" ; 
2043 mtg_cluster_sc:cluster_size "80"^^xsd:int ;

2044

$2045:$ :pL17

2046 a skos:Concept ;

2047 mtg_cluster_sc:id "pCL17" ;

2048 skos:broader :pCL80 ;

2049 rdfs:label "PCDH20-expressing cerebral cortex MTG GABAergic interneuron" ;

2050 mtg_cluster_sc:evidence "Inh L1-2 VIP PCDH20" ;

2051 go_sc:part_of uberon:UBERON_0002771;

2052 mtg_cluster_sc:enriched_in "cortical_layer2" ;

2053 mtg_cluster_sc:has_soma_location_in "cortical_layer1" ;

2054 mtg_cluster_sc:has_soma_location_in "cortical_layer2" ;

2055 mtg_cluster_sc:selectively_expresses hugo:HGNC_14257 ;

2056 mtg_cluster_sc:neuron_type "GABAergic" ;

2057 mtg_cluster_sc:cluster_size "61"^^xsd:int ;

2058

2059 :pCL18

2060 a skos:Concept ;

2061 mtg_cluster_sc:id "pCL18" ;

2062 skos:broader :pCL80 ;

2063 rdfs:label "SERPINF1-expressing cerebral cortex MTG GABAergic interneuron" ;

2064 mtg_cluster_sc:evidence "Inh L2-5 VIP SERPINF1" ;

2065 go_sc:part_of uberon:UBERON_0002771;

2066 mtg_cluster_sc:enriched_in "cortical_layer4" ;

2067 mtg_cluster_sc:has_soma_location_in "cortical_layer2" ;

2068 mtg_cluster_sc:has_soma_location_in "cortical_layer3" ;

2069 mtg_cluster_sc:has_soma_location_in "cortical_layer4" ;

2070 mtg_cluster_sc:has_soma_location_in "cortical_layer5" ;

2071 mtg_cluster_sc:selectively_expresses hugo:HGNC_8824 ; 
2072 mtg_cluster_sc:neuron_type "GABAergic" ;

2073 mtg_cluster_sc:cluster_size "55"^^xsd:int ;

2074 .

$2075:$ :PC19

2076 a skos:Concept ;

2077 mtg_cluster_sc:id "pCL19" ;

2078 skos:broader :pCL80 ;

2079 rdfs:label "TYR-expressing cerebral cortex MTG GABAergic interneuron" ;

2080 mtg_cluster_sc:evidence "Inh L2-5 VIP TYR" ;

2081 go_sc:part_of uberon:UBERON_0002771 ;

2082 mtg_cluster_sc:enriched_in "cortical_layer4" ;

2083 mtg_cluster_sc:has_soma_location_in "cortical_layer2" ;

2084 mtg_cluster_sc:has_soma_location_in "cortical_layer3" ;

2085 mtg_cluster_sc:has_soma_location_in "cortical_layer4" ;

2086 mtg_cluster_sc:has_soma_location_in "cortical_layer5" ;

2087 mtg_cluster_sc:selectively_expresses hugo:HGNC_12442 ;

2088 mtg_cluster_sc:neuron_type "GABAergic" ;

2089 mtg_cluster_sc:cluster_size "62"^^xsd:int ;

2090 .

2091 :pCL20

2092 a skos:Concept ;

2093 mtg_cluster_sc:id "pCL20" ;

2094 skos:broader :pCL80 ;

2095 rdfs:label "CHRM2-expressing cerebral cortex MTG GABAergic interneuron" ;

2096 mtg_cluster_sc:evidence "Inh L1-3 VIP CHRM2" ;

2097 go_sc:part_of uberon:UBERON_0002771 ;

2098 mtg_cluster_sc:enriched_in "cortical_layer2" ;

2099 mtg_cluster_sc:has_soma_location_in "cortical_layer1" ;

2100 mtg_cluster_sc:has_soma_location_in "cortical_layer2" ; 
2101 mtg_cluster_sc:has_soma_location_in "cortical_layer3" ;

2102 mtg_cluster_sc:selectively_expresses hugo:HGNC_1951;

2103 mtg_cluster_sc:neuron_type "GABAergic" ;

2104 mtg_cluster_sc:cluster_size "175"^^xsd:int ;

2105

$2106:$ :pCL21

2107 a skos:Concept ;

2108 mtg_cluster_sc:id "pCL21" ;

2109 skos:broader :pCL80 ;

2110 rdfs:label "CBLN1-expressing cerebral cortex MTG GABAergic interneuron" ;

2111 mtg_cluster_sc:evidence "Inh L2-4 VIP CBLN1" ;

2112 go_sc:part_of uberon:UBERON_0002771 ;

2113 mtg_cluster_sc:enriched_in "cortical_layer3" ;

2114 mtg_cluster_sc:has_soma_location_in "cortical_layer2" ;

2115 mtg_cluster_sc:has_soma_location_in "cortical_layer3" ;

2116 mtg_cluster_sc:has_soma_location_in "cortical_layer4" ;

2117 mtg_cluster_sc:selectively_expresses hugo:HGNC_1543;

2118 mtg_cluster_sc:neuron_type "GABAergic" ;

2119 mtg_cluster_sc:cluster_size "67"^^xsd:int ;

$2120 \quad$.

2121 :pCL22

2122 a skos:Concept ;

2123 mtg_cluster_sc:id "pCL22" ;

$2124 \quad$ skos:broader :pCL80 ;

2125 rdfs:label "CCDC184-expressing cerebral cortex MTG GABAergic interneuron" ;

2126 mtg_cluster_sc:evidence "Inh L1-3 VIP CCDC184" ;

2127 go_sc:part_of uberon:UBERON_0002771 ;

2128 mtg_cluster_sc:enriched_in "cortical_layer2" ;

2129 mtg_cluster_sc:has_soma_location_in "cortical_layer1" ; 
2130 mtg_cluster_sc:has_soma_location_in "cortical_layer2" ;

2131 mtg_cluster_sc:has_soma_location_in "cortical_layer3" ;

2132 mtg_cluster_sc:selectively_expresses hugo:HGNC_33749 ;

2133 mtg_cluster_sc:neuron_type "GABAergic" ;

2134 mtg_cluster_sc:cluster_size "64"^^xsd:int ;

2135

2136 :pCL23

2137 a skos:Concept ;

2138 mtg_cluster_sc:id "pCL23" ;

2139 skos:broader :pCL80 ;

2140 rdfs:label "GGH-expressing cerebral cortex MTG GABAergic interneuron" ;

2141 mtg_cluster_sc:evidence "Inh L1-3 VIP GGH" ;

2142 go_sc:part_of uberon:UBERON_0002771;

2143 mtg_cluster_sc:enriched_in "cortical_layer2" ;

2144 mtg_cluster_sc:has_soma_location_in "cortical_layer1" ;

2145 mtg_cluster_sc:has_soma_location_in "cortical_layer2" ;

2146 mtg_cluster_sc:has_soma_location_in "cortical_layer3" ;

2147 mtg_cluster_sc:selectively_expresses hugo:HGNC_4248;

2148 mtg_cluster_sc:neuron_type "GABAergic" ;

2149 mtg_cluster_sc:cluster_size "68"^^xsd:int ;

2150

$2151 \quad$ :pCL24

2152 a skos:Concept ;

2153 mtg_cluster_sc:id "pCL24" ;

2154 skos:broader :pCL80 ;

2155 rdfs:label "LBH-expressing cerebral cortex MTG GABAergic interneuron" ;

2156 mtg_cluster_sc:evidence "Inh L1-2 VIP LBH" ;

2157 go_sc:part_of uberon:UBERON_0002771 ;

2158 mtg_cluster_sc:enriched_in "cortical_layer2" ; 
2159 mtg_cluster_sc:has_soma_location_in "cortical_layer1" ;

2160 mtg_cluster_sc:has_soma_location_in "cortical_layer2" ;

2161 mtg_cluster_sc:selectively_expresses hugo:HGNC_29532 ;

2162 mtg_cluster_sc:neuron_type "GABAergic" ;

2163 mtg_cluster_sc:cluster_size "47"^^xsd:int ;

2164

2165 :pCL25

2166 a skos:Concept ;

2167 mtg_cluster_sc:id "pCL25" ;

2168 skos:broader :pCL80 ;

2169 rdfs:label "CASC6-expressing cerebral cortex MTG GABAergic interneuron" ;

2170 mtg_cluster_sc:evidence "Inh L2-3 VIP CASC6" ;

2171 go_sc:part_of uberon:UBERON_0002771;

2172 mtg_cluster_sc:enriched_in "cortical_layer2" ;

2173 mtg_cluster_sc:has_soma_location_in "cortical_layer2" ;

2174 mtg_cluster_sc:has_soma_location_in "cortical_layer3" ;

2175 mtg_cluster_sc:selectively_expresses hugo:HGNC_49076 ;

2176 mtg_cluster_sc:neuron_type "GABAergic" ;

2177 mtg_cluster_sc:cluster_size "45"^^xsd:int ;

2178

2179 :pCL26

2180 a skos:Concept ;

2181 mtg_cluster_sc:id "pCL26" ;

$2182 \quad$ skos:broader :pCL80 ;

2183 rdfs:label "SPAG17-expressing cerebral cortex MTG GABAergic interneuron" ;

2184 mtg_cluster_sc:evidence "Inh L2-4 VIP SPAG17" ;

2185 go_sc:part_of uberon:UBERON_0002771;

2186 mtg_cluster_sc:enriched_in "cortical_layer3" ;

2187 mtg_cluster_sc:has_soma_location_in "cortical_layer2" ; 
2188 mtg_cluster_sc:has_soma_location_in "cortical_layer3" ;

2189 mtg_cluster_sc:has_soma_location_in "cortical_layer4" ;

2190 mtg_cluster_sc:selectively_expresses hugo:HGNC_26620 ;

2191 mtg_cluster_sc:neuron_type "GABAergic" ;

2192 mtg_cluster_sc:cluster_size "33"^^xsd:int ;

2193.

2194 :pCL27

2195 a skos:Concept ;

2196 mtg_cluster_sc:id "pCL27" ;

2197 skos:broader :pCL80 ;

2198 rdfs:label "OPRM1-expressing cerebral cortex MTG GABAergic interneuron" ;

2199 mtg_cluster_sc:evidence "Inh L1-4 VIP OPRM1" ;

2200 go_sc:part_of uberon:UBERON_0002771;

2201 mtg_cluster_sc:enriched_in "cortical_layer3" ;

2202 mtg_cluster_sc:has_soma_location_in "cortical_layer1" ;

2203 mtg_cluster_sc:has_soma_location_in "cortical_layer2" ;

2204 mtg_cluster_sc:has_soma_location_in "cortical_layer3" ;

2205 mtg_cluster_sc:has_soma_location_in "cortical_layer4" ;

2206 mtg_cluster_sc:selectively_expresses hugo:HGNC_8156 ;

2207 mtg_cluster_sc:neuron_type "GABAergic" ;

2208 mtg_cluster_sc:cluster_size "52"^^xsd:int ;

2209

2210 :pCL28

2211 a skos:Concept ;

2212 mtg_cluster_sc:id "pCL28" ;

2213 skos:broader :pCL81;

2214 rdfs:label "NPY-expressing cerebral cortex MTG GABAergic interneuron" ;

2215 mtg_cluster_sc:evidence "Inh L3-6 SST NPY" ;

2216 go_sc:part_of uberon:UBERON_0002771; 
2217 mtg_cluster_sc:enriched_in "cortical_layer5" ;

2218 mtg_cluster_sc:has_soma_location_in "cortical_layer3" ;

2219 mtg_cluster_sc:has_soma_location_in "cortical_layer4" ;

2220 mtg_cluster_sc:has_soma_location_in "cortical_layer5" ;

2221 mtg_cluster_sc:has_soma_location_in "cortical_layer6" ;

2222 mtg_cluster_sc:selectively_expresses hugo:HGNC_7955 ;

2223 mtg_cluster_sc:neuron_type "GABAergic" ;

2224 mtg_cluster_sc:cluster_size "15"^^xsd:int ;

2225

2226 :pCL29

2227 a skos:Concept ;

2228 mtg_cluster_sc:id "pCL29" ;

2229 skos:broader :pCL81;

2230 rdfs:label "HPGD-expressing cerebral cortex MTG GABAergic interneuron" ;

2231 mtg_cluster_sc:evidence "Inh L3-6 SST HPGD" ;

2232 go_sc:part_of uberon:UBERON_0002771;

2233 mtg_cluster_sc:enriched_in "cortical_layer5" ;

2234 mtg_cluster_sc:has_soma_location_in "cortical_layer3" ;

2235 mtg_cluster_sc:has_soma_location_in "cortical_layer4" ;

2236 mtg_cluster_sc:has_soma_location_in "cortical_layer5" ;

2237 mtg_cluster_sc:has_soma_location_in "cortical_layer6" ;

2238 mtg_cluster_sc:selectively_expresses hugo:HGNC_5154 ;

2239 mtg_cluster_sc:neuron_type "GABAergic" ;

2240 mtg_cluster_sc:cluster_size "60"^^xsd:int ;

2241

$2242: p C L 30$

2243 a skos:Concept ;

2244 mtg_cluster_sc:id "pCL30" ;

$2245 \quad$ skos:broader :pCL81; 
2246 rdfs:label "B3GAT2-expressing cerebral cortex MTG GABAergic interneuron" ;

2247 mtg_cluster_sc:evidence "Inh L4-6 SST B3GAT2" ;

2248 go_sc:part_of uberon:UBERON_0002771 ;

2249 mtg_cluster_sc:enriched_in "cortical_layer5" ;

2250 mtg_cluster_sc:has_soma_location_in "cortical_layer4" ;

2251 mtg_cluster_sc:has_soma_location_in "cortical_layer5" ;

2252 mtg_cluster_sc:has_soma_location_in "cortical_layer6" ;

2253 mtg_cluster_sc:selectively_expresses hugo:HGNC_922 ;

2254 mtg_cluster_sc:neuron_type "GABAergic" ;

2255 mtg_cluster_sc:cluster_size "182"^^xsd:int ;

2256

2257 :pCL31

2258 a skos:Concept ;

2259 mtg_cluster_sc:id "pCL31" ;

2260 skos:broader :pCL81;

2261 rdfs:label "KLHDC8A-expressing cerebral cortex MTG GABAergic interneuron" ;

2262 mtg_cluster_sc:evidence "Inh L5-6 SST KLHDC8A" ;

2263 go_sc:part_of uberon:UBERON_0002771 ;

2264 mtg_cluster_sc:enriched_in "cortical_layer5" ;

2265 mtg_cluster_sc:has_soma_location_in "cortical_layer5" ;

2266 mtg_cluster_sc:has_soma_location_in "cortical_layer6" ;

2267 mtg_cluster_sc:selectively_expresses hugo:HGNC_25573 ;

2268 mtg_cluster_sc:neuron_type "GABAergic" ;

2269 mtg_cluster_sc:cluster_size "63"^^xsd:int ;

2270 .

$2271:$ : $C L 32$

2272 a skos:Concept ;

2273 mtg_cluster_sc:id "pCL32" ;

2274 skos:broader :pCL81; 
2275 rdfs:label "NPM1P10-expressing cerebral cortex MTG GABAergic interneuron" ;

2276 mtg_cluster_sc:evidence "Inh L5-6 SST NPM1P10" ;

2277 go_sc:part_of uberon:UBERON_0002771 ;

2278 mtg_cluster_sc:enriched_in "cortical_layer5" ;

2279 mtg_cluster_sc:has_soma_location_in "cortical_layer5" ;

2280 mtg_cluster_sc:has_soma_location_in "cortical_layer6" ;

2281 mtg_cluster_sc:selectively_expresses hugo:HGNC_7912 ;

2282 mtg_cluster_sc:neuron_type "GABAergic" ;

2283 mtg_cluster_sc:cluster_size "79"^^xsd:int ;

2285 :pCL33

2286 a skos:Concept ;

2287 mtg_cluster_sc:id "pCL33" ;

2288 skos:broader :pCL81;

2289 rdfs:label "GXYLT2-expressing cerebral cortex MTG GABAergic interneuron" ;

2290 mtg_cluster_sc:evidence "Inh L4-6 SST GXYLT2" ;

2291 go_sc:part_of uberon:UBERON_0002771 ;

2292 mtg_cluster_sc:enriched_in "cortical_layer5" ;

2293 mtg_cluster_sc:has_soma_location_in "cortical_layer4" ;

2294 mtg_cluster_sc:has_soma_location_in "cortical_layer5" ;

2295 mtg_cluster_sc:has_soma_location_in "cortical_layer6" ;

2296 mtg_cluster_sc:selectively_expresses hugo:HGNC_33383 ;

2297 mtg_cluster_sc:neuron_type "GABAergic" ;

2298 mtg_cluster_sc:cluster_size "41"^^xsd:int ;

2299 .

2300 :pCL34

2301 a skos:Concept ;

2302 mtg_cluster_sc:id "pCL34" ;

2303 skos:broader :pCL81; 
2304 rdfs:label "STK32A-expressing cerebral cortex MTG GABAergic interneuron" ;

2305 mtg_cluster_sc:evidence "Inh L4-5 SST STK32A" ;

2306 go_sc:part_of uberon:UBERON_0002771 ;

2307 mtg_cluster_sc:enriched_in "cortical_layer4" ;

2308 mtg_cluster_sc:has_soma_location_in "cortical_layer4" ;

2309 mtg_cluster_sc:has_soma_location_in "cortical_layer5" ;

2310 mtg_cluster_sc:selectively_expresses hugo:HGNC_28317 ;

2311 mtg_cluster_sc:neuron_type "GABAergic" ;

2312 mtg_cluster_sc:cluster_size "93"^^xsd:int ;

2314 :pCL35

2315 a skos:Concept ;

2316 mtg_cluster_sc:id "pCL35" ;

$2317 \quad$ skos:broader :pCL81;

2318 rdfs:label "CALB1-expressing cerebral cortex MTG GABAergic interneuron" ;

2319 mtg_cluster_sc:evidence "Inh L1-3 SST CALB1" ;

2320 go_sc:part_of uberon:UBERON_0002771;

2321 mtg_cluster_sc:enriched_in "cortical_layer2" ;

2322 mtg_cluster_sc:has_soma_location_in "cortical_layer1" ;

2323 mtg_cluster_sc:has_soma_location_in "cortical_layer2" ;

2324 mtg_cluster_sc:has_soma_location_in "cortical_layer3" ;

2325 mtg_cluster_sc:selectively_expresses hugo:HGNC_1434 ;

2326 mtg_cluster_sc:neuron_type "GABAergic" ;

2327 mtg_cluster_sc:cluster_size "279"^^xsd:int ;

2328.

2329 :pCL36

2330 a skos:Concept ;

2331 mtg_cluster_sc:id "pCL36" ;

$2332 \quad$ skos:broader :pCL81; 
2333 rdfs:label "ADGRG6-expressing cerebral cortex MTG GABAergic interneuron" ;

2334 mtg_cluster_sc:evidence "Inh L3-5 SST ADGRG6" ;

2335 go_sc:part_of uberon:UBERON_0002771 ;

2336 mtg_cluster_sc:enriched_in "cortical_layer4" ;

2337 mtg_cluster_sc:has_soma_location_in "cortical_layer3" ;

2338 mtg_cluster_sc:has_soma_location_in "cortical_layer4" ;

2339 mtg_cluster_sc:has_soma_location_in "cortical_layer5" ;

2340 mtg_cluster_sc:selectively_expresses hugo:HGNC_13841;

2341 mtg_cluster_sc:neuron_type "GABAergic" ;

2342 mtg_cluster_sc:cluster_size "132"^^xsd:int ;

2343.

$2344:$ :pC37

2345 a skos:Concept ;

2346 mtg_cluster_sc:id "pCL37" ;

2347 skos:broader :pCL81;

2348 rdfs:label "FRZB-expressing cerebral cortex MTG GABAergic interneuron" ;

2349 mtg_cluster_sc:evidence "Inh L2-4 SST FRZB" ;

2350 go_sc:part_of uberon:UBERON_0002771 ;

2351 mtg_cluster_sc:enriched_in "cortical_layer3" ;

2352 mtg_cluster_sc:has_soma_location_in "cortical_layer2" ;

2353 mtg_cluster_sc:has_soma_location_in "cortical_layer3" ;

2354 mtg_cluster_sc:has_soma_location_in "cortical_layer4" ;

2355 mtg_cluster_sc:selectively_expresses hugo:HGNC_3959 ;

2356 mtg_cluster_sc:neuron_type "GABAergic" ;

2357 mtg_cluster_sc:cluster_size "64"^^xsd:int ;

2358 .

$2359:$ :pL38

2360 a skos:Concept ;

2361 mtg_cluster_sc:id "pCL38" ; 
2362 skos:broader :pCL81;

2363 rdfs:label "TH-expressing-expressing cerebral cortex MTG GABAergic interneuron" ;

2364 mtg_cluster_sc:evidence "Inh L5-6 SST TH" ;

2365 go_sc:part_of uberon:UBERON_0002771;

2366 mtg_cluster_sc:enriched_in "cortical_layer5" ;

2367 mtg_cluster_sc:has_soma_location_in "cortical_layer5" ;

2368 mtg_cluster_sc:has_soma_location_in "cortical_layer6" ;

2369 mtg_cluster_sc:selectively_expresses hugo:HGNC_11782 ;

2370 mtg_cluster_sc:neuron_type "GABAergic" ;

2371 mtg_cluster_sc:cluster_size "27"^^xsd:int ;

2372 .

$2373:$ :pL39

2374 a skos:Concept ;

2375 mtg_cluster_sc:id "pCL39" ;

2376 skos:broader :pCL82 ;

2377 rdfs:label "GLP1R-expressing cerebral cortex MTG GABAergic interneuron" ;

2378 mtg_cluster_sc:evidence "Inh L5-6 GAD1 GLP1R" ;

2379 go_sc:part_of uberon:UBERON_0002771;

2380 mtg_cluster_sc:enriched_in "cortical_layer6" ;

2381 mtg_cluster_sc:has_soma_location_in "cortical_layer5" ;

2382 mtg_cluster_sc:has_soma_location_in "cortical_layer6" ;

2383 mtg_cluster_sc:selectively_expresses hugo:HGNC_4324 ;

2384 mtg_cluster_sc:neuron_type "GABAergic" ;

2385 mtg_cluster_sc:cluster_size "27"^^xsd:int ;

2386 .

2387 :pCL40

2388 a skos:Concept ;

2389 mtg_cluster_sc:id "pCL40" ;

2390 skos:broader :pCL82 ; 
2391 rdfs:label "LGR5-expressing cerebral cortex MTG GABAergic interneuron" ;

2392 mtg_cluster_sc:evidence "Inh L5-6 PVALB LGR5" ;

2393 go_sc:part_of uberon:UBERON_0002771 ;

2394 mtg_cluster_sc:enriched_in "cortical_layer5" ;

2395 mtg_cluster_sc:has_soma_location_in "cortical_layer5" ;

2396 mtg_cluster_sc:has_soma_location_in "cortical_layer6" ;

2397 mtg_cluster_sc:selectively_expresses hugo:HGNC_4504 ;

2398 mtg_cluster_sc:neuron_type "GABAergic" ;

2399 mtg_cluster_sc:cluster_size "52"^^xsd:int ;

2400

$2401:$ :pCL41

2402 a skos:Concept ;

2403 mtg_cluster_sc:id "pCL41" ;

$2404 \quad$ skos:broader :pCL82 ;

2405 rdfs:label "MEPE-expressing cerebral cortex MTG GABAergic interneuron" ;

2406 mtg_cluster_sc:evidence "Inh L4-5 PVALB MEPE" ;

2407 go_sc:part_of uberon:UBERON_0002771;

2408 mtg_cluster_sc:enriched_in "cortical_layer5" ;

2409 mtg_cluster_sc:has_soma_location_in "cortical_layer4" ;

2410 mtg_cluster_sc:has_soma_location_in "cortical_layer5" ;

2411 mtg_cluster_sc:selectively_expresses hugo:HGNC_13361;

2412 mtg_cluster_sc:neuron_type "GABAergic" ;

2413 mtg_cluster_sc:cluster_size "64"^^xsd:int ;

2415 :pCL42

2416 a skos:Concept ;

2417 mtg_cluster_sc:id "pCL42" ;

2418 skos:broader :pCL82 ;

2419 rdfs:label "WFDC2-expressing cerebral cortex MTG GABAergic interneuron" ; 
2420 mtg_cluster_sc:evidence "Inh L2-4 PVALB WFDC2" ;

2421 go_sc:part_of uberon:UBERON_0002771;

2422 mtg_cluster_sc:enriched_in "cortical_layer3" ;

2423 mtg_cluster_sc:has_soma_location_in "cortical_layer2" ;

2424 mtg_cluster_sc:has_soma_location_in "cortical_layer3" ;

2425 mtg_cluster_sc:has_soma_location_in "cortical_layer4" ;

2426 mtg_cluster_sc:selectively_expresses hugo:HGNC_15939;

2427 mtg_cluster_sc:neuron_type "GABAergic" ;

2428 mtg_cluster_sc:cluster_size "387"^^xsd:int ;

$2430:$ : 2443

2431 a skos:Concept ;

2432 mtg_cluster_sc:id "pCL43" ;

2433 skos:broader :pCL82;

2434 rdfs:label "SULF1-expressing cerebral cortex MTG GABAergic interneuron" ;

2435 mtg_cluster_sc:evidence "Inh L4-6 PVALB SULF1" ;

2436 go_sc:part_of uberon:UBERON_0002771;

2437 mtg_cluster_sc:enriched_in "cortical_layer5" ;

2438 mtg_cluster_sc:has_soma_location_in "cortical_layer4" ;

2439 mtg_cluster_sc:has_soma_location_in "cortical_layer5" ;

2440 mtg_cluster_sc:has_soma_location_in "cortical_layer6" ;

2441 mtg_cluster_sc:selectively_expresses hugo:HGNC_20391;

2442 mtg_cluster_sc:neuron_type "GABAergic" ;

2443 mtg_cluster_sc:cluster_size "167"^^xsd:int ;

2444 .

2445 :pCL44

2446 a skos:Concept ;

2447 mtg_cluster_sc:id "pCL44" ;

$2448 \quad$ skos:broader :pCL82 ; 
2449 rdfs:label "SST|MIR548F2-expressing cerebral cortex MTG GABAergic interneuron" ;

2450 mtg_cluster_sc:evidence "Inh L5-6 SST MIR548F2" ;

2451 go_sc:part_of uberon:UBERON_0002771 ;

2452 mtg_cluster_sc:enriched_in "cortical_layer5" ;

2453 mtg_cluster_sc:has_soma_location_in "cortical_layer5" ;

2454 mtg_cluster_sc:has_soma_location_in "cortical_layer6" ;

2455 mtg_cluster_sc:selectively_expresses hugo:HGNC_11329 ;

2456 mtg_cluster_sc:selectively_expresses hugo:HGNC_35306 ;

2457 mtg_cluster_sc:neuron_type "GABAergic" ;

2458 mtg_cluster_sc:cluster_size "80"^^xsd:int ;

2459

$2460:$ :pL45

2461 a skos:Concept ;

2462 mtg_cluster_sc:id "pCL45" ;

2463 skos:broader :pCL82 ;

2464 rdfs:label "SCUBE3-expressing cerebral cortex MTG GABAergic interneuron" ;

2465 mtg_cluster_sc:evidence "Inh L2-5 PVALB SCUBE3" ;

2466 go_sc:part_of uberon:UBERON_0002771 ;

2467 mtg_cluster_sc:enriched_in "cortical_layer3" ;

2468 mtg_cluster_sc:has_soma_location_in "cortical_layer2" ;

2469 mtg_cluster_sc:has_soma_location_in "cortical_layer3" ;

2470 mtg_cluster_sc:has_soma_location_in "cortical_layer4" ;

2471 mtg_cluster_sc:has_soma_location_in "cortical_layer5" ;

2472 mtg_cluster_sc:selectively_expresses hugo:HGNC_13655 ;

2473 mtg_cluster_sc:neuron_type "GABAergic" ;

2474 mtg_cluster_sc:cluster_size "32"^^xsd:int ;

2475

$2476:$ :pL46

2477 a skos:Concept ; 
2478 mtg_cluster_sc:id "pCL46" ;

2479 skos:broader :pCL77 ;

2480 rdfs:label "LAMP5|LTK-expressing cerebral cortex MTG Glutamatergic neuron" ;

2481 mtg_cluster_sc:evidence "Exc L2 LAMP5 LTK" ;

2482 go_sc:part_of uberon:UBERON_0002771;

2483 mtg_cluster_sc:enriched_in "cortical_layer2" ;

2484 mtg_cluster_sc:has_soma_location_in "cortical_layer2" ;

2485 mtg_cluster_sc:selectively_expresses hugo:HGNC_16097 ;

2486 mtg_cluster_sc:selectively_expresses hugo:HGNC_6721;

2487 mtg_cluster_sc:neuron_type "Glutamatergic" ;

2488 mtg_cluster_sc:cluster_size "812"^^xsd:int ;

2489 .

$2490:$ :pCL47

2491 a skos:Concept ;

2492 mtg_cluster_sc:id "pCL47" ;

2493 skos:broader :pCL77 ;

2494 rdfs:label "LINC00507|GLP2R-expressing cerebral cortex MTG Glutamatergic neuron" ;

2495 mtg_cluster_sc:evidence "Exc L2-4 LINC00507 GLP2R" ;

2496 go_sc:part_of uberon:UBERON_0002771 ;

2497 mtg_cluster_sc:enriched_in "cortical_layer3" ;

2498 mtg_cluster_sc:has_soma_location_in "cortical_layer2" ;

2499 mtg_cluster_sc:has_soma_location_in "cortical_layer3" ;

2500 mtg_cluster_sc:has_soma_location_in "cortical_layer4" ;

2501 mtg_cluster_sc:selectively_expresses hugo:HGNC_43558;

2502 mtg_cluster_sc:selectively_expresses hugo:HGNC_4325 ;

2503 mtg_cluster_sc:neuron_type "Glutamatergic" ;

2504 mtg_cluster_sc:cluster_size "170"^^xsd:int ;

2505

2506 :pCL48 
2507 a skos:Concept ;

2508 mtg_cluster_sc:id "pCL48" ;

2509 skos:broader :pCL77 ;

2510 rdfs:label "LINC00507|FREM3-expressing cerebral cortex MTG Glutamatergic neuron" ;

2511 mtg_cluster_sc:evidence "Exc L2-3 LINC00507 FREM3" ;

2512 go_sc:part_of uberon:UBERON_0002771;

2513 mtg_cluster_sc:enriched_in "cortical_layer3" ;

2514 mtg_cluster_sc:has_soma_location_in "cortical_layer2" ;

2515 mtg_cluster_sc:has_soma_location_in "cortical_layer3" ;

2516 mtg_cluster_sc:selectively_expresses hugo:HGNC_43558;

2517 mtg_cluster_sc:selectively_expresses hugo:HGNC_25172 ;

2518 mtg_cluster_sc:neuron_type "Glutamatergic" ;

2519 mtg_cluster_sc:cluster_size "2284"^^xsd:int ;

2520

$2521:$ :pC49

2522 a skos:Concept ;

2523 mtg_cluster_sc:id "pCL49" ;

2524 skos:broader :pCL77 ;

2525 rdfs:label "THEMIS|C1QL3-expressing cerebral cortex MTG Glutamatergic neuron" ;

2526 mtg_cluster_sc:evidence "Exc L5-6 THEMIS C1QL3" ;

2527 go_sc:part_of uberon:UBERON_0002771 ;

2528 mtg_cluster_sc:enriched_in "cortical_layer6" ;

2529 mtg_cluster_sc:has_soma_location_in "cortical_layer5" ;

2530 mtg_cluster_sc:has_soma_location_in "cortical_layer6" ;

2531 mtg_cluster_sc:selectively_expresses hugo:HGNC_21569 ;

2532 mtg_cluster_sc:selectively_expresses hugo:HGNC_19359;

2533 mtg_cluster_sc:neuron_type "Glutamatergic" ;

2534 mtg_cluster_sc:cluster_size "1537"^^xsd:int ; 
2536 :pCL50

2537 a skos:Concept ;

2538 mtg_cluster_sc:id "pCL50" ;

2539 skos:broader :pCL77 ;

2540 rdfs:label "RORB|CARM1P1-expressing cerebral cortex MTG Glutamatergic neuron" ;

2541 mtg_cluster_sc:evidence "Exc L3-4 RORB CARM1P1" ;

2542 go_sc:part_of uberon:UBERON_0002771 ;

2543 mtg_cluster_sc:enriched_in "cortical_layer3" ;

2544 mtg_cluster_sc:has_soma_location_in "cortical_layer3" ;

2545 mtg_cluster_sc:has_soma_location_in "cortical_layer4" ;

2546 mtg_cluster_sc:selectively_expresses hugo:HGNC_10259 ;

2547 mtg_cluster_sc:selectively_expresses hugo:HGNC_23392 ;

2548 mtg_cluster_sc:neuron_type "Glutamatergic" ;

2549 mtg_cluster_sc:cluster_size "280"^^xsd:int ;

2550 .

2551 :pCL51

2552 a skos:Concept ;

2553 mtg_cluster_sc:id "pCL51" ;

$2554 \quad$ skos:broader :pCL77 ;

2555 rdfs:label "RORB|ESR1-expressing cerebral cortex MTG Glutamatergic neuron" ;

2556 mtg_cluster_sc:evidence "Exc L3-5 RORB ESR1" ;

2557 go_sc:part_of uberon:UBERON_0002771 ;

2558 mtg_cluster_sc:enriched_in "cortical_layer4" ;

2559 mtg_cluster_sc:has_soma_location_in "cortical_layer3" ;

2560 mtg_cluster_sc:has_soma_location_in "cortical_layer4" ;

2561 mtg_cluster_sc:has_soma_location_in "cortical_layer5" ;

2562 mtg_cluster_sc:selectively_expresses hugo:HGNC_10259 ;

2563 mtg_cluster_sc:selectively_expresses hugo:HGNC_3467 ;

2564 mtg_cluster_sc:neuron_type "Glutamatergic" ; 
2565 mtg_cluster_sc:cluster_size "1428"^^xsd:int ;

2566 .

2567 :pCL52

2568 a skos:Concept ;

2569 mtg_cluster_sc:id "pCL52" ;

$2570 \quad$ skos:broader :pCL77 ;

2571 rdfs:label "RORB|COL22A1-expressing cerebral cortex MTG Glutamatergic neuron" ;

2572 mtg_cluster_sc:evidence "Exc L3-5 RORB COL22A1" ;

2573 go_sc:part_of uberon:UBERON_0002771;

2574 mtg_cluster_sc:enriched_in "cortical_layer4" ;

2575 mtg_cluster_sc:has_soma_location_in "cortical_layer3" ;

2576 mtg_cluster_sc:has_soma_location_in "cortical_layer4" ;

2577 mtg_cluster_sc:has_soma_location_in "cortical_layer5" ;

2578 mtg_cluster_sc:selectively_expresses hugo:HGNC_10259;

2579 mtg_cluster_sc:selectively_expresses hugo:HGNC_22989 ;

2580 mtg_cluster_sc:neuron_type "Glutamatergic" ;

2581 mtg_cluster_sc:cluster_size "160"^^xsd:int ;

2582 .

2583 :pCL53

2584 a skos:Concept ;

2585 mtg_cluster_sc:id "pCL53" ;

2586 skos:broader :pCL77 ;

2587 rdfs:label "RORB|FILIP1L-expressing cerebral cortex MTG Glutamatergic neuron" ;

2588 mtg_cluster_sc:evidence "Exc L3-5 RORB FILIP1L" ;

2589 go_sc:part_of uberon:UBERON_0002771 ;

2590 mtg_cluster_sc:enriched_in "cortical_layer4" ;

2591 mtg_cluster_sc:has_soma_location_in "cortical_layer3" ;

2592 mtg_cluster_sc:has_soma_location_in "cortical_layer4" ;

2593 mtg_cluster_sc:has_soma_location_in "cortical_layer5" ; 
2594 mtg_cluster_sc:selectively_expresses hugo:HGNC_10259 ;

2595 mtg_cluster_sc:selectively_expresses hugo:HGNC_24589;

2596 mtg_cluster_sc:neuron_type "Glutamatergic" ;

2597 mtg_cluster_sc:cluster_size "153"^^xsd:int ;

2598

2599 :pCL54

2600 a skos:Concept ;

2601 mtg_cluster_sc:id "pCL54" ;

2602 skos:broader :pCL77;

2603 rdfs:label "RORB|TWIST2-expressing cerebral cortex MTG Glutamatergic neuron" ;

2604 mtg_cluster_sc:evidence "Exc L3-5 RORB TWIST2" ;

2605 go_sc:part_of uberon:UBERON_0002771 ;

2606 mtg_cluster_sc:enriched_in "cortical_layer4" ;

2607 mtg_cluster_sc:has_soma_location_in "cortical_layer3" ;

2608 mtg_cluster_sc:has_soma_location_in "cortical_layer4" ;

2609 mtg_cluster_sc:has_soma_location_in "cortical_layer5" ;

2610 mtg_cluster_sc:selectively_expresses hugo:HGNC_10259;

2611 mtg_cluster_sc:selectively_expresses hugo:HGNC_20670 ;

2612 mtg_cluster_sc:neuron_type "Glutamatergic" ;

2613 mtg_cluster_sc:cluster_size "93"^^xsd:int ;

2614

2615 :pCL55

2616 a skos:Concept ;

2617 mtg_cluster_sc:id "pCL55" ;

$2618 \quad$ skos:broader :pCL77 ;

2619 rdfs:label "RORB|FOLH1B-expressing cerebral cortex MTG Glutamatergic neuron" ;

2620 mtg_cluster_sc:evidence "Exc L4-5 RORB FOLH1B" ;

2621 go_sc:part_of uberon:UBERON_0002771 ;

2622 mtg_cluster_sc:enriched_in "cortical_layer4" ; 
2623 mtg_cluster_sc:has_soma_location_in "cortical_layer4" ;

2624 mtg_cluster_sc:has_soma_location_in "cortical_layer5" ;

2625 mtg_cluster_sc:selectively_expresses hugo:HGNC_10259 ;

2626 mtg_cluster_sc:selectively_expresses hugo:HGNC_13636 ;

2627 mtg_cluster_sc:neuron_type "Glutamatergic" ;

2628 mtg_cluster_sc:cluster_size "870"^^xsd:int ;

2629

2630 :pCL56

2631 a skos:Concept ;

2632 mtg_cluster_sc:id "pCL56" ;

2633 skos:broader :pCL77 ;

2634 rdfs:label "RORB|SEMA3E-expressing cerebral cortex MTG Glutamatergic neuron" ;

2635 mtg_cluster_sc:evidence "Exc L4-6 RORB SEMA3E" ;

2636 go_sc:part_of uberon:UBERON_0002771;

2637 mtg_cluster_sc:enriched_in "cortical_layer5" ;

2638 mtg_cluster_sc:has_soma_location_in "cortical_layer4" ;

2639 mtg_cluster_sc:has_soma_location_in "cortical_layer5" ;

2640 mtg_cluster_sc:has_soma_location_in "cortical_layer6" ;

2641 mtg_cluster_sc:selectively_expresses hugo:HGNC_10259 ;

2642 mtg_cluster_sc:selectively_expresses hugo:HGNC_10727 ;

2643 mtg_cluster_sc:neuron_type "Glutamatergic" ;

2644 mtg_cluster_sc:cluster_size "777"^^xsd:int ;

2645 .

2646 :pCL57

2647 a skos:Concept ;

2648 mtg_cluster_sc:id "pCL57" ;

2649 skos:broader :pCL77 ;

2650 rdfs:label "RORB|DAPK2-expressing cerebral cortex MTG Glutamatergic neuron" ;

2651 mtg_cluster_sc:evidence "Exc L4-5 RORB DAPK2" ; 
2652 go_sc:part_of uberon:UBERON_0002771 ;

2653 mtg_cluster_sc:enriched_in "cortical_layer4" ;

2654 mtg_cluster_sc:has_soma_location_in "cortical_layer4" ;

2655 mtg_cluster_sc:has_soma_location_in "cortical_layer5" ;

2656 mtg_cluster_sc:selectively_expresses hugo:HGNC_10259 ;

2657 mtg_cluster_sc:selectively_expresses hugo:HGNC_2675 ;

2658 mtg_cluster_sc:neuron_type "Glutamatergic" ;

2659 mtg_cluster_sc:cluster_size "173"^^xsd:int ;

2660 .

$2661: p C L 58$

2662 a skos:Concept ;

2663 mtg_cluster_sc:id "pCL58" ;

2664 skos:broader :pCL77 ;

2665 rdfs:label "RORB|TTC12-expressing cerebral cortex MTG Glutamatergic neuron" ;

2666 mtg_cluster_sc:evidence "Exc L5-6 RORB TTC12" ;

2667 go_sc:part_of uberon:UBERON_0002771 ;

2668 mtg_cluster_sc:enriched_in "cortical_layer5" ;

2669 mtg_cluster_sc:has_soma_location_in "cortical_layer5" ;

2670 mtg_cluster_sc:has_soma_location_in "cortical_layer6" ;

2671 mtg_cluster_sc:selectively_expresses hugo:HGNC_10259 ;

2672 mtg_cluster_sc:selectively_expresses hugo:HGNC_23700 ;

2673 mtg_cluster_sc:neuron_type "Glutamatergic" ;

2674 mtg_cluster_sc:cluster_size "167"^^xsd:int ;

2675

2676 :pCL59

2677 a skos:Concept ;

2678 mtg_cluster_sc:id "pCL59" ;

2679 skos:broader :pCL77 ;

2680 rdfs:label "RORB|C1R-expressing cerebral cortex MTG Glutamatergic neuron" ; 
2681 mtg_cluster_sc:evidence "Exc L4-6 RORB C1R" ;

2682 go_sc:part_of uberon:UBERON_0002771 ;

2683 mtg_cluster_sc:enriched_in "cortical_layer5" ;

2684 mtg_cluster_sc:has_soma_location_in "cortical_layer4" ;

2685 mtg_cluster_sc:has_soma_location_in "cortical_layer5" ;

2686 mtg_cluster_sc:has_soma_location_in "cortical_layer6" ;

2687 mtg_cluster_sc:selectively_expresses hugo:HGNC_10259 ;

2688 mtg_cluster_sc:selectively_expresses hugo:HGNC_1246 ;

2689 mtg_cluster_sc:neuron_type "Glutamatergic" ;

2690 mtg_cluster_sc:cluster_size "160"^^xsd:int ;

2691

$2692:$ :pL60

2693 a skos:Concept ;

2694 mtg_cluster_sc:id "pCL60" ;

2695 skos:broader :pCL77 ;

2696 rdfs:label "FEZF2|SCN4B-expressing cerebral cortex MTG Glutamatergic neuron" ;

2697 mtg_cluster_sc:evidence "Exc L4-5 FEZF2 SCN4B" ;

2698 go_sc:part_of uberon:UBERON_0002771;

2699 mtg_cluster_sc:enriched_in "cortical_layer5" ;

2700 mtg_cluster_sc:has_soma_location_in "cortical_layer4" ;

2701 mtg_cluster_sc:has_soma_location_in "cortical_layer5" ;

2702 mtg_cluster_sc:selectively_expresses hugo:HGNC_13506 ;

2703 mtg_cluster_sc:selectively_expresses hugo:HGNC_10592 ;

2704 mtg_cluster_sc:neuron_type "Glutamatergic" ;

2705 mtg_cluster_sc:cluster_size "25"^^xsd:int ;

2707 :pCL61

2708 a skos:Concept ;

2709 mtg_cluster_sc:id "pCL61" ; 
$2710 \quad$ skos:broader :pCL77 ;

2711 rdfs:label "THEMIS|DCSTAMP-expressing cerebral cortex MTG Glutamatergic neuron" ;

2712 mtg_cluster_sc:evidence "Exc L5-6 THEMIS DCSTAMP" ;

2713 go_sc:part_of uberon:UBERON_0002771 ;

2714 mtg_cluster_sc:enriched_in "cortical_layer5" ;

2715 mtg_cluster_sc:has_soma_location_in "cortical_layer5" ;

2716 mtg_cluster_sc:has_soma_location_in "cortical_layer6" ;

2717 mtg_cluster_sc:selectively_expresses hugo:HGNC_21569 ;

2718 mtg_cluster_sc:selectively_expresses hugo:HGNC_18549 ;

2719 mtg_cluster_sc:neuron_type "Glutamatergic" ;

2720 mtg_cluster_sc:cluster_size "53"^^xsd:int ;

2721 .

2722 :pCL62

2723 a skos:Concept ;

2724 mtg_cluster_sc:id "pCL62" ;

2725 skos:broader :pCL77 ;

2726 rdfs:label "THEMIS|CRABP1-expressing cerebral cortex MTG Glutamatergic neuron" ;

2727 mtg_cluster_sc:evidence "Exc L5-6 THEMIS CRABP1" ;

2728 go_sc:part_of uberon:UBERON_0002771;

2729 mtg_cluster_sc:enriched_in "cortical_layer5" ;

2730 mtg_cluster_sc:has_soma_location_in "cortical_layer5" ;

2731 mtg_cluster_sc:has_soma_location_in "cortical_layer6" ;

2732 mtg_cluster_sc:selectively_expresses hugo:HGNC_21569;

2733 mtg_cluster_sc:selectively_expresses hugo:HGNC_2338;

2734 mtg_cluster_sc:neuron_type "Glutamatergic" ;

2735 mtg_cluster_sc:cluster_size "147"^^xsd:int ;

2736 .

2737 :pCL63

2738 a skos:Concept ; 
2739 mtg_cluster_sc:id "pCL63" ;

$2740 \quad$ skos:broader :pCL77 ;

2741 rdfs:label "THEMIS|FGF10-expressing cerebral cortex MTG Glutamatergic neuron" ;

2742 mtg_cluster_sc:evidence "Exc L5-6 THEMIS FGF10" ;

2743 go_sc:part_of uberon:UBERON_0002771;

2744 mtg_cluster_sc:enriched_in "cortical_layer5" ;

2745 mtg_cluster_sc:has_soma_location_in "cortical_layer5" ;

2746 mtg_cluster_sc:has_soma_location_in "cortical_layer6" ;

2747 mtg_cluster_sc:selectively_expresses hugo:HGNC_21569 ;

2748 mtg_cluster_sc:selectively_expresses hugo:HGNC_3666 ;

2749 mtg_cluster_sc:neuron_type "Glutamatergic" ;

2750 mtg_cluster_sc:cluster_size "78"^^xsd:int ;

2751

2752 :pCL64

2753 a skos:Concept ;

2754 mtg_cluster_sc:id "pCL64" ;

2755 skos:broader :pCL77 ;

2756 rdfs:label "FEZF2||L26-expressing cerebral cortex MTG Glutamatergic neuron" ;

2757 mtg_cluster_sc:evidence "Exc L4-6 FEZF2 IL26" ;

2758 go_sc:part_of uberon:UBERON_0002771;

2759 mtg_cluster_sc:enriched_in "cortical_layer5" ;

2760 mtg_cluster_sc:has_soma_location_in "cortical_layer4" ;

2761 mtg_cluster_sc:has_soma_location_in "cortical_layer5" ;

2762 mtg_cluster_sc:has_soma_location_in "cortical_layer6" ;

2763 mtg_cluster_sc:selectively_expresses hugo:HGNC_13506 ;

2764 mtg_cluster_sc:selectively_expresses hugo:HGNC_17119;

2765 mtg_cluster_sc:neuron_type "Glutamatergic" ;

2766 mtg_cluster_sc:cluster_size " $344^{\text {"^^} x s d: i n t ~ ; ~}$ 
2768 :pCL65

2769 a skos:Concept ;

2770 mtg_cluster_sc:id "pCL65" ;

2771 skos:broader :pCL77 ;

2772 rdfs:label "FEZF2|ABO-expressing cerebral cortex MTG Glutamatergic neuron" ;

2773 mtg_cluster_sc:evidence "Exc L5-6 FEZF2 ABO" ;

2774 go_sc:part_of uberon:UBERON_0002771 ;

2775 mtg_cluster_sc:enriched_in "cortical_layer6" ;

2776 mtg_cluster_sc:has_soma_location_in "cortical_layer5" ;

2777 mtg_cluster_sc:has_soma_location_in "cortical_layer6" ;

2778 mtg_cluster_sc:selectively_expresses hugo:HGNC_13506 ;

2779 mtg_cluster_sc:selectively_expresses hugo:HGNC_79 ;

2780 mtg_cluster_sc:neuron_type "Glutamatergic" ;

2781 mtg_cluster_sc:cluster_size "373"^^xsd:int ;

2782 .

2783 :pCL66

2784 a skos:Concept ;

2785 mtg_cluster_sc:id "pCL66" ;

$2786 \quad$ skos:broader :pCL77 ;

2787 rdfs:label "FEZF2|SCUBE1-expressing cerebral cortex MTG Glutamatergic neuron" ;

2788 mtg_cluster_sc:evidence "Exc L6 FEZF2 SCUBE1" ;

2789 go_sc:part_of uberon:UBERON_0002771;

2790 mtg_cluster_sc:enriched_in "cortical_layer6" ;

2791 mtg_cluster_sc:has_soma_location_in "cortical_layer6" ;

2792 mtg_cluster_sc:selectively_expresses hugo:HGNC_13506 ;

2793 mtg_cluster_sc:selectively_expresses hugo:HGNC_13441;

2794 mtg_cluster_sc:neuron_type "Glutamatergic" ;

2795 mtg_cluster_sc:cluster_size "52"^^xsd:int ; 
2797 :pCL67

2798 a skos:Concept ;

2799 mtg_cluster_sc:id "pCL67" ;

$2800 \quad$ skos:broader :pCL77 ;

2801 rdfs:label "IL15-expressing cerebral cortex MTG Glutamatergic neuron" ;

2802 mtg_cluster_sc:evidence "Exc L5-6 SLC17A7 IL15" ;

2803 go_sc:part_of uberon:UBERON_0002771;

2804 mtg_cluster_sc:enriched_in "cortical_layer6" ;

2805 mtg_cluster_sc:has_soma_location_in "cortical_layer5" ;

2806 mtg_cluster_sc:has_soma_location_in "cortical_layer6" ;

2807 mtg_cluster_sc:selectively_expresses hugo:HGNC_5977 ;

2808 mtg_cluster_sc:neuron_type "Glutamatergic" ;

2809 mtg_cluster_sc:cluster_size "56"^^xsd:int ;

2810

2811 :pCL68

2812 a skos:Concept ;

2813 mtg_cluster_sc:id "pCL68" ;

$2814 \quad$ skos:broader :pCL77;

2815 rdfs:label "FEZF2|OR2T8-expressing cerebral cortex MTG Glutamatergic neuron" ;

2816 mtg_cluster_sc:evidence "Exc L6 FEZF2 OR2T8" ;

2817 go_sc:part_of uberon:UBERON_0002771 ;

2818 mtg_cluster_sc:enriched_in "cortical_layer6" ;

2819 mtg_cluster_sc:has_soma_location_in "cortical_layer6" ;

2820 mtg_cluster_sc:selectively_expresses hugo:HGNC_13506 ;

2821 mtg_cluster_sc:selectively_expresses hugo:HGNC_15020 ;

2822 mtg_cluster_sc:neuron_type "Glutamatergic" ;

2823 mtg_cluster_sc:cluster_size "19"^^xsd:int ;

2824

2825 :pCL69 
a skos:Concept ;

2827 mtg_cluster_sc:id "pCL69" ;

2828 skos:broader :pCL77 ;

2829 rdfs:label "FEZF2|EFTUD1P1-expressing cerebral cortex MTG Glutamatergic neuron" ;

2830 mtg_cluster_sc:evidence "Exc L5-6 FEZF2 EFTUD1P1" ;

2831 go_sc:part_of uberon:UBERON_0002771 ;

2832 mtg_cluster_sc:enriched_in "cortical_layer6" ;

2833 mtg_cluster_sc:has_soma_location_in "cortical_layer5" ;

2834 mtg_cluster_sc:has_soma_location_in "cortical_layer6" ;

2835 mtg_cluster_sc:selectively_expresses hugo:HGNC_13506 ;

2836 mtg_cluster_sc:selectively_expresses hugo:HGNC_31739 ;

2837 mtg_cluster_sc:neuron_type "Glutamatergic" ;

2838 mtg_cluster_sc:cluster_size "314"^^xsd:int ;

2839 .

$2840:$ :pC70

2841 a skos:Concept ;

2842 mtg_cluster_sc:id "pCL70" ;

2843 skos:broader :pCL83 ;

2844 rdfs:label "PDGFRA-expressing MTG Oligodendrocyte precursor cell" ;

2845 mtg_cluster_sc:evidence "OPC L1-6 PDGFRA" ;

2846 go_sc:part_of uberon:UBERON_0002771;

2847 mtg_cluster_sc:enriched_in "cortical_layer4" ;

2848 mtg_cluster_sc:has_soma_location_in "cortical_layer1" ;

2849 mtg_cluster_sc:has_soma_location_in "cortical_layer2" ;

2850 mtg_cluster_sc:has_soma_location_in "cortical_layer3" ;

2851 mtg_cluster_sc:has_soma_location_in "cortical_layer4" ;

2852 mtg_cluster_sc:has_soma_location_in "cortical_layer5" ;

2853 mtg_cluster_sc:has_soma_location_in "cortical_layer6" ;

2854 mtg_cluster_sc:selectively_expresses hugo:HGNC_8803 ; 
2855 mtg_cluster_sc:cluster_size "238"^^xsd:int ;

2856

2857 :pCL71

2858 a skos:Concept ;

2859 mtg_cluster_sc:id "pCL71" ;

2860 skos:broader :pCL89 ;

2861 rdfs:label "SLC14A1-expressing MTG astrocyte" ;

2862 mtg_cluster_sc:evidence "Astro L1-6 FGFR3 SLC14A1" ;

2863 go_sc:part_of uberon:UBERON_0002771;

2864 mtg_cluster_sc:enriched_in "cortical_layer3" ;

2865 mtg_cluster_sc:has_soma_location_in "cortical_layer1" ;

2866 mtg_cluster_sc:has_soma_location_in "cortical_layer2" ;

2867 mtg_cluster_sc:has_soma_location_in "cortical_layer3" ;

2868 mtg_cluster_sc:has_soma_location_in "cortical_layer4" ;

2869 mtg_cluster_sc:has_soma_location_in "cortical_layer5" ;

2870 mtg_cluster_sc:has_soma_location_in "cortical_layer6" ;

2871 mtg_cluster_sc:selectively_expresses hugo:HGNC_10918 ;

2872 mtg_cluster_sc:cluster_size "230"^^xsd:int ;

2873 .

$2874 \quad$ :pCL72

2875 a skos:Concept ;

2876 mtg_cluster_sc:id "pCL72" ;

2877 skos:broader :pCL89;

2878 rdfs:label "GFAP-expressing MTG astrocyte" ;

2879 mtg_cluster_sc:evidence "Astro L1-2 FGFR3 GFAP" ;

2880 go_sc:part_of uberon:UBERON_0002771 ;

2881 mtg_cluster_sc:enriched_in "cortical_layer2" ;

2882 mtg_cluster_sc:has_soma_location_in "cortical_layer1" ;

2883 mtg_cluster_sc:has_soma_location_in "cortical_layer2" ; 
2884 mtg_cluster_sc:selectively_expresses hugo:HGNC_4235;

2885 mtg_cluster_sc:cluster_size "61"^^xsd:int ;

2886

$2887:$ :pL73

2888 a skos:Concept ;

2889 mtg_cluster_sc:id "pCL73" ;

2890 skos:broader :pCL86 ;

2891 rdfs:label "OPALIN-expressing MTG Oligodendrocyte" ;

2892 mtg_cluster_sc:evidence "Oligo L1-6 OPALIN" ;

2893 go_sc:part_of uberon:UBERON_0002771;

2894 mtg_cluster_sc:enriched_in "cortical_layer5" ;

2895 mtg_cluster_sc:has_soma_location_in "cortical_layer1" ;

2896 mtg_cluster_sc:has_soma_location_in "cortical_layer2" ;

2897 mtg_cluster_sc:has_soma_location_in "cortical_layer3" ;

2898 mtg_cluster_sc:has_soma_location_in "cortical_layer4" ;

2899 mtg_cluster_sc:has_soma_location_in "cortical_layer5" ;

2900 mtg_cluster_sc:has_soma_location_in "cortical_layer6" ;

2901 mtg_cluster_sc:selectively_expresses hugo:HGNC_20707 ;

2902 mtg_cluster_sc:cluster_size "313"^^xsd:int ;

2903

2904 :pCL74

2905 a skos:Concept ;

2906 mtg_cluster_sc:id "pCL74" ;

$2907 \quad$ skos:broader :pCL87 ;

2908 rdfs:label "NOSTRIN-expressing cerebral cortex MTG endothelial cell" ;

2909 mtg_cluster_sc:evidence "Endo L2-6 NOSTRIN" ;

2910 go_sc:part_of uberon:UBERON_0002771;

2911 mtg_cluster_sc:enriched_in "cortical_layer4" ;

2912 mtg_cluster_sc:has_soma_location_in "cortical_layer2" ; 
2913 mtg_cluster_sc:has_soma_location_in "cortical_layer3" ;

2914 mtg_cluster_sc:has_soma_location_in "cortical_layer4" ;

2915 mtg_cluster_sc:has_soma_location_in "cortical_layer5" ;

2916 mtg_cluster_sc:has_soma_location_in "cortical_layer6" ;

2917 mtg_cluster_sc:selectively_expresses hugo:HGNC_20203 ;

2918 mtg_cluster_sc:cluster_size "9"^^xsd:int ;

2919 .

$2920:$ :pCL75

2921 a skos:Concept ;

2922 mtg_cluster_sc:id "pCL75" ;

$2923 \quad$ skos:broader :pCL88;

2924 rdfs:label "TYROBP-expressing MTG Microglial cell" ;

2925 mtg_cluster_sc:evidence "Micro L1-3 TYROBP" ;

2926 go_sc:part_of uberon:UBERON_0002771;

2927 mtg_cluster_sc:enriched_in "cortical_layer3" ;

2928 mtg_cluster_sc:has_soma_location_in "cortical_layer1" ;

2929 mtg_cluster_sc:has_soma_location_in "cortical_layer2" ;

2930 mtg_cluster_sc:has_soma_location_in "cortical_layer3" ;

2931 mtg_cluster_sc:selectively_expresses hugo:HGNC_12449 ;

2932 mtg_cluster_sc:cluster_size "63"^^xsd:int ;

2933

2934 :pCL76

2935 a skos:Concept ;

2936 mtg_cluster_sc:id "pCL76" ;

2937 skos:broader :pCL90 ;

2938 rdfs:label "GAD1-expressing cerebral cortex MTG GABAergic interneuron" ;

2939 go_sc:part_of uberon:UBERON_0002771;

2940 mtg_cluster_sc:selectively_expresses hugo:HGNC_4092 ;

2941 mtg_cluster_sc:neuron_type "GABAergic" ; 
2943 :pCL77

2944 a skos:Concept ;

2945 mtg_cluster_sc:id "pCL77" ;

2946 skos:broader :pCL91;

2947 rdfs:label "SLC17A7-expressing MTG Glutamatergic neuron" ;

2948 go_sc:part_of uberon:UBERON_0002771 ;

2949 mtg_cluster_sc:selectively_expresses hugo:HGNC_16704 ;

2950 mtg_cluster_sc:neuron_type "Glutamatergic" ;

2951 .

2952 :pCL78

2953 a skos:Concept ;

2954 mtg_cluster_sc:id "pCL78" ;

2955 skos:broader :pCL76 ;

2956 rdfs:label "ADARB2-expressing cerebral cortex MTG GABAergic interneuron" ;

2957 go_sc:part_of uberon:UBERON_0002771 ;

2958 mtg_cluster_sc:selectively_expresses hugo:HGNC_227 ;

2959 mtg_cluster_sc:neuron_type "GABAergic" ;

2960 .

2961 :pCL79

2962 a skos:Concept ;

2963 mtg_cluster_sc:id "pCL79" ;

2964 skos:broader :pCL76 ;

2965 rdfs:label "LHX6-expressing cerebral cortex MTG GABAergic interneuron" ;

2966 go_sc:part_of uberon:UBERON_0002771 ;

2967 mtg_cluster_sc:selectively_expresses hugo:HGNC_21735 ;

2968 mtg_cluster_sc:neuron_type "GABAergic" ;

2969 .

2970 :pCL80 
2971 a skos:Concept ;

2972 mtg_cluster_sc:id "pCL80" ;

2973 skos:broader :pCL78 ;

2974 rdfs:label "VIP-expressing cerebral cortex MTG GABAergic interneuron" ;

2975 go_sc:part_of uberon:UBERON_0002771 ;

2976 mtg_cluster_sc:selectively_expresses hugo:HGNC_12693 ;

2977 mtg_cluster_sc:neuron_type "GABAergic" ;

2978 .

2979 :pCL81

2980 a skos:Concept ;

2981 mtg_cluster_sc:id "pCL81" ;

2982 skos:broader :pCL79;

2983 rdfs:label "SST-expressing cerebral cortex MTG GABAergic interneuron" ;

2984 go_sc:part_of uberon:UBERON_0002771 ;

2985 mtg_cluster_sc:selectively_expresses hugo:HGNC_11329 ;

2986 mtg_cluster_sc:neuron_type "GABAergic" ;

2987 .

2988 :pCL82

2989 a skos:Concept ;

2990 mtg_cluster_sc:id "pCL82" ;

2991 skos:broader :pCL79;

2992 rdfs:label "PVALB-expressing cerebral cortex MTG GABAergic interneuron" ;

2993 go_sc:part_of uberon:UBERON_0002771;

2994 mtg_cluster_sc:selectively_expresses hugo:HGNC_9704 ;

2995 mtg_cluster_sc:neuron_type "GABAergic" ;

2996 .

2997 :pCL83

2998 a skos:Concept ;

2999 mtg_cluster_sc:id "pCL83" ; 
3000 skos:broader cl:CL_0002453 ;

3001 rdfs:label "MTG Oligodendrocyte precursor cell" ;

3002 go_sc:part_of uberon:UBERON_0002771 ;

3003 .

3004 :pCL84

3005 a skos:Concept ;

3006 mtg_cluster_sc:id "pCL84" ;

3007 skos:broader cl:CL_0002605 ;

3008 rdfs:label "MTG Astrocyte of the cerebral cortex" ;

3009 go_sc:part_of uberon:UBERON_0002771 ;

3010 .

3011 :pCL86

3012 a skos:Concept ;

3013 mtg_cluster_sc:id "pCL86" ;

3014 skos:broader cl:CL_0000128;

3015 rdfs:label "MTG Oligodendrocyte" ;

3016 go_sc:part_of uberon:UBERON_0002771 ;

3017 .

3018 :pCL87

3019 a skos:Concept ;

3020 mtg_cluster_sc:id "pCL87" ;

3021 skos:broader cl:CL_1001602 ;

3022 rdfs:label "cerebral cortex MTG endothelial cell" ;

3023 go_sc:part_of uberon:UBERON_0002771 ;

3024 .

3025 :pCL88

3026 a skos:Concept ;

3027 mtg_cluster_sc:id "pCL88" ;

3028 skos:broader cl:CL_0000129; 
3029 rdfs:label "MTG Microglial cell" ;

3030 go_sc:part_of uberon:UBERON_0002771 ;

3031 .

3032 :pCL89

3033 a skos:Concept ;

3034 mtg_cluster_sc:id "pCL89" ;

3035 skos:broader :pCL84 ;

3036 rdfs:label "FGFR3-expressing MTG astrocyte" ;

3037 go_sc:part_of uberon:UBERON_0002771 ;

3038 mtg_cluster_sc:selectively_expresses hugo:HGNC_3690 ;

3039

3040 :pCL90

3041 a skos:Concept ;

3042 mtg_cluster_sc:id "pCL90" ;

3043 skos:broader cl:CL_0010011;

3044 rdfs:label "cerebral cortex MTG GABAergic interneuron" ;

3045 go_sc:part_of uberon:UBERON_0002771;

3046 mtg_cluster_sc:selectively_expresses hugo:HGNC_4092 ;

3047 mtg_cluster_sc:neuron_type "GABAergic" ;

3048 .

3049 :pCL91

3050 a skos:Concept ;

3051 mtg_cluster_sc:id "pCL91" ;

3052 skos:broader cl:CL_0000679;

3053 rdfs:label "MTG Glutamatergic neuron" ;

3054 go_sc:part_of uberon:UBERON_0002771 ;

3055 mtg_cluster_sc:selectively_expresses hugo:HGNC_16704 ;

3056 mtg_cluster_sc:neuron_type "Glutamatergic" ; 